\title{
Access to Cyclic Amino Boronates via Rhodium-Catalyzed Functionalization of Alkyl MIDA-Boronates
}

\author{
Jeffrey D. St. Denis, C. Frank Lee, and Andrei K. Yudin* \\ Davenport Research Laboratories, Department of Chemistry, \\ University of Toronto, 80 St. George Street, Toronto, Ontario, Canada M5S 3H6 \\ Supporting Information
}

\section{Contents}

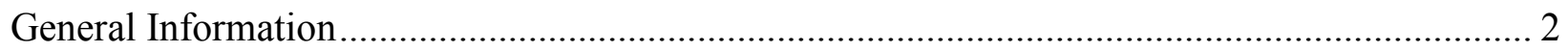

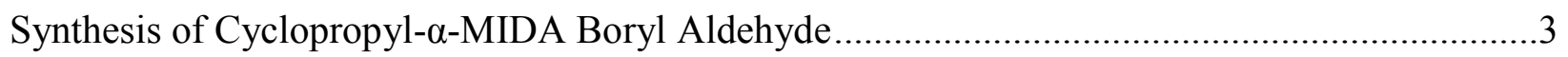

Synthesis and Scope of the Reduction of Boryl Aldehydes to Boryl Alcohols...............................6

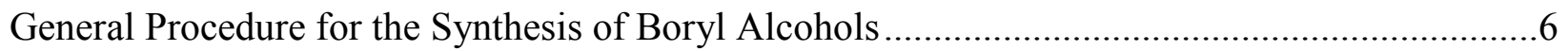

General Procedure for the Synthesis of $\alpha$-Boryl Sulfamate Esters .............................................13

Characterization Data for $\alpha$-Boryl Formate Esters ……........................................................19

General Procedure for the Rh-Catalyzed C-H-Amination of $\alpha$-Boryl Sulfamate Esters ................21

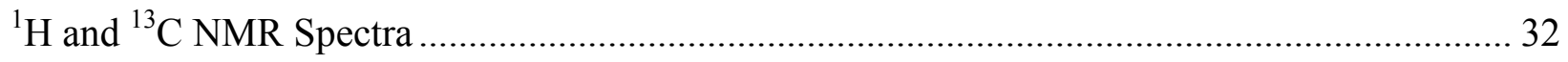




\section{General Information}

Tetrahydrofuran (THF) was purified by distillation from sodium/benzophenone ketyl radical under an atmosphere of nitrogen. Acetonitrile $(\mathrm{MeCN})$ was purified by distillation from $3 \AA$ molecular sieves ( $3 \AA \mathrm{MS})$. Dichloromethane, methanol $(\mathrm{MeOH})$ and triethylamine $\left(\mathrm{Et}_{3} \mathrm{~N}\right)$ were purified by distillation from $\mathrm{CaH}_{2}$ respectively. Diethyl ether $\left(\mathrm{Et}_{2} \mathrm{O}\right)$, toluene, dimethyl formamide (DMF), was purified through a solvent purification system. ${ }^{1}$ All other reagents were purchased from commercial sources used as received.

\section{Chromatography}

Flash column chromatography was carried out using Silicycle 230-400 mesh silica gel. Thinlayer chromatography (TLC) was performed on Macherey Nagel pre-coated glass backed TLC plates (SIL G/UV254, $0.25 \mathrm{~mm}$ ) and visualized using a UV lamp (254 nm) $\mathrm{KMnO}_{4}$, ninhydrin, or $\mathrm{I}_{2}$ stain in case of no $\mathrm{UV}$ activity.

\section{Nuclear Magnetic Resonance Spectroscopy}

Proton ( $\left({ }^{1} \mathrm{H}-\mathrm{NMR}\right)$, carbon $\left({ }^{13} \mathrm{C}-\mathrm{NMR}\right)$ and 2D NMR experiments were performed on Bruker 400 $\mathrm{MHz}$ or Varian 300,400 , or $500 \mathrm{MHz}$ spectrometers. NMR spectra chemical shifts $(\delta)$ are reported in parts per million (ppm) referenced to residual protonated solvent peak $\left(\mathrm{CDCl}_{3}, \delta=\right.$ 7.26, DMSO- $d_{6}, \delta=2.49$, acetone- $d_{6} \delta=2.05$, acetonitrile- $d_{3} \delta=1.94$ ). Spectral data is reported as follows: chemical shift, multiplicity $(\mathrm{s}=$ singlet, $\mathrm{d}=$ doublet, $\mathrm{t}=$ triplet, $\mathrm{q}=$ quartet, $\mathrm{dd}=$ doublet of doublets, $\mathrm{dt}=$ doublet of triplets, $\mathrm{ddt}=$ doublet of doublet of triplets, $\mathrm{dtd}=$ doublet of triplet of doublets, $\mathrm{m}=$ multiplet, $\mathrm{br}=$ broad), coupling constant $(J)$ in $\operatorname{Hertz}(\mathrm{Hz})$, and integration. ${ }^{13} \mathrm{C}$ NMR spectra chemical shifts $(\delta)$ are reported in parts per million (ppm) were referenced to carbon resonances in the NMR solvent $\left(\mathrm{CDCl}_{3}, \delta=77.0\right.$; DMSO- $d_{6}, \delta=39.5$, center line, acetone- $d_{6}, \delta=206.2,29.8$ (centre line), acetonitrile- $d_{3}, \delta=118.2,1.3$ (centre line)). High-Resolution Mass Spectra was obtained at the University of Toronto mass spectrometry centre.

${ }^{1}$ Pangborn, A.B.; Giardello, M.A.; Grubbs, R.H. Organometallics 1996, 5, 1518-1520. 


\section{Infrared Spectroscopy}

IR spectra were recorded on a Perkin-Elmer 100 instrument equipped with a single-reflection diamond/ZnSe ATR accessory. Performed on a $\mathrm{NaCl}$ disc as a thin layer.

\section{Mass Spectrometry}

High resolution mass spectra were obtained on a VG 70- 250S (double focusing) mass spectrometer at $70 \mathrm{eV}$ or on an $\mathrm{ABI} / \mathrm{Sciex}$ Qstar mass spectrometer with ESI source, MS/MS and accurate mass capabilities.

\section{Synthesis of Cyclopropyl- $\alpha$-MIDA Boryl Aldehyde}

To an oven dried, stir bar quipped vial was added alkyne ( $660 \mathrm{mg}, 10 \mathrm{mmol}, 1.0$ equiv) followed by the dropwise addition of neat catechol borane $(22 \mathrm{mmol})$. The reaction flask was then warmed to $65^{\circ} \mathrm{C}$ for $24 \mathrm{hrs}$. Upon complete consumption of starting alkene the reaction was removed from the reaction block and allowed to cool to room temperature. Then distilled water $(10 \mathrm{~mL})$ was added to the reaction mixture and allowed to stir for 2-3 hrs at which time the solid precipitate was filtered to yield the crude boronic acid.

The crude boronic acid was then dissolved in DMF (0.3 M) and $N$-methyliminodiacetic acid (1.1 equiv) was added. The mixture was warmed to $80{ }^{\circ} \mathrm{C}$ for 24 hours. The reaction was then removed from heat and concentrated in vacuo. To the residue was added $50 \mathrm{~mL} \mathrm{H}_{2} \mathrm{O}, 50 \mathrm{~mL}$ sat. $\mathrm{NaHCO}_{3}$ and $100 \mathrm{~mL}$ EtOAc and the organic was then separated. The aqueous layer was the extracted 5 x $75 \mathrm{~mL}$ EtOAc. The combined organics were washed with brine, dried over $\mathrm{NaSO}_{4}$ and concentrated to yield crude MIDA boronate. The crude MIDA boronate was then purified via $\mathrm{SiO}_{2}$ using hexanes:acetone to yield pure primary alkyl MIDA boronate as a white solid. ${ }^{2}$

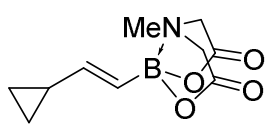

(E)-(2-cyclopropylvinyl)MIDA boronate

$1.82 \mathrm{~g}, 82 \%$, white solid

2 Grob, J. E.; Nunez, J.; Dechantsreiter, M. A; Hamann, L. G. J. Org. Chem. 2011, 76, 4930-40. 
${ }^{1} \mathrm{H}$ NMR (399 MHz, Acetonitrile- $d_{3}$ )

$\delta 5.58(\mathrm{dd}, J=17.6,8.4 \mathrm{~Hz}, 1 \mathrm{H}), 5.47(\mathrm{~d}, J=17.6 \mathrm{~Hz}, 1 \mathrm{H}), 3.93(\mathrm{~d}, J=16.9 \mathrm{~Hz}, 2 \mathrm{H})$, $3.77(\mathrm{~d}, J=16.9 \mathrm{~Hz}, 2 \mathrm{H}), 2.78(\mathrm{~s}, 3 \mathrm{H}), 1.50(\mathrm{~m}, 1 \mathrm{H}), 0.82-0.70(\mathrm{~m}, 2 \mathrm{H}), 0.52-0.38$ $(\mathrm{m}, 2 \mathrm{H})$

${ }^{13} \mathrm{C}$ NMR $\left(101 \mathrm{MHz}\right.$, Acetonitrile- $\left.d_{3}\right)$

$\delta 168.0,149.3,60.9,46.2,15.7,6.2$

${ }^{11}$ B NMR (128 MHz, Acetonitrile- $d_{3}$ )

$\delta 10.6$

HRMS [DART-MS] $\left(\mathrm{M}+\mathrm{H}^{+}\right)$

$m / z$ calculated for $\mathrm{C}_{10} \mathrm{H}_{15} \mathrm{BNO}_{4}=224.1094$

$m / z$ found $=224.1097$

TLC (hexanes:acetone 1:1)

$$
\mathrm{R}_{\mathrm{f}}=0.38
$$

\section{Epoxidation/Rearrangement}

To an oven dried round bottom flask equipped with a magnetic stir was charged cyclopropyl vinyl MIDA boronate $(1.82 \mathrm{~g}, 8.2 \mathrm{mmol}, 1.0$ equiv) followed by a $0.06 \mathrm{M}$ solution of dimethyldioxirane in acetone ( 2.5 equiv). The mixture was allowed to stir for overnight at room temperature, at which time ${ }^{1} \mathrm{H}$ NMR analysis showed complete consumption of starting alkene. The reaction was then concentrated in vacuo to yield a white solid. The solid was then dissolved in a mixture of toluene and $\mathrm{MeCN}$ (approx. 1:1) and evaporated under reduced pressure (five times) to azeotrope the residual water. The solid was then evacuated under high-vacuum for at least 1 hour.

The solid was then suspended in DCM $(0.05 \mathrm{M})$ and cooled to $-40{ }^{\circ} \mathrm{C}$ with a MeCN-dry ice bath. $\mathrm{Next}, \mathrm{BF}_{3} \cdot \mathrm{OEt}_{2}$ (1.25 equiv) was added dropwise over the course of five minutes. Upon complete addition of the $\mathrm{BF}_{3} \cdot \mathrm{OEt}_{2}$ the flask was transferred to an ice-water bath and allowed to stir for another 45 minutes. The reaction was then quenched with the addition of sat. NaHCO3, transferred to a separatory funnel and extracted with EtOAc (5x). The combined organics were then dried over anhydrous $\mathrm{Na}_{2} \mathrm{SO}_{4}$, filtered and concentrated to yield a white residue. The crude 
material was then purified via $\mathrm{SiO}_{2}$ purification to yield the cyclopropyl aldehyde as a white solid.

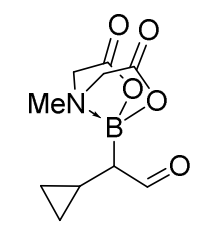

$\alpha$-MIDA-cyclopropyl boryl aldehyde

$1.38 \mathrm{~g}, 71 \%$ yield, white solid

${ }^{1} \mathrm{H}$ NMR (500 MHz, Acetone- $d_{6}$ )

$\delta 9.80(\mathrm{~d}, J=3.0 \mathrm{~Hz}, 1 \mathrm{H}), 4.30(\mathrm{~d}, J=17.1 \mathrm{~Hz}, 1 \mathrm{H}), 4.25(\mathrm{~d}, J=16.8 \mathrm{~Hz}, 1 \mathrm{H}), 4.09$ (d, $J$

$=17.1 \mathrm{~Hz}, 1 \mathrm{H}), 4.04(\mathrm{~d}, J=16.8 \mathrm{~Hz}, 1 \mathrm{H}), 3.18(\mathrm{~s}, 3 \mathrm{H}), 1.61-1.54(\mathrm{~m}, 1 \mathrm{H}), 1.04(\mathrm{~m}$, $1 \mathrm{H}), 0.66-0.55(\mathrm{~m}, 2 \mathrm{H}), 0.32-0.26(\mathrm{~m}, 1 \mathrm{H}), 0.13-0.06(\mathrm{~m}, 1 \mathrm{H})$

${ }^{13} \mathrm{C}$ NMR (126 MHz, Acetone- $d_{6}$ )

$\delta 206.2,167.6,167.3,62.5,62.2,46.0,7.0,4.8,4.2$

${ }^{11}$ B NMR (128 MHz, Acetone- $d_{6}$ )

$\delta 11.4$

HRMS [DART-MS] $\left(\mathrm{M}+\mathrm{H}^{+}\right)$

$m / z$ calculated for $\mathrm{C}_{10} \mathrm{H}_{15} \mathrm{BNO}_{5}=240.1043$

$\mathrm{m} / \mathrm{z}$ found $=240.1042$

TLC (hexanes:acetone 1:2)

$$
\mathrm{R}_{\mathrm{f}}=0.4
$$




\section{Synthesis and Scope of the Reduction of Boryl Aldehydes to Boryl Alcohols}
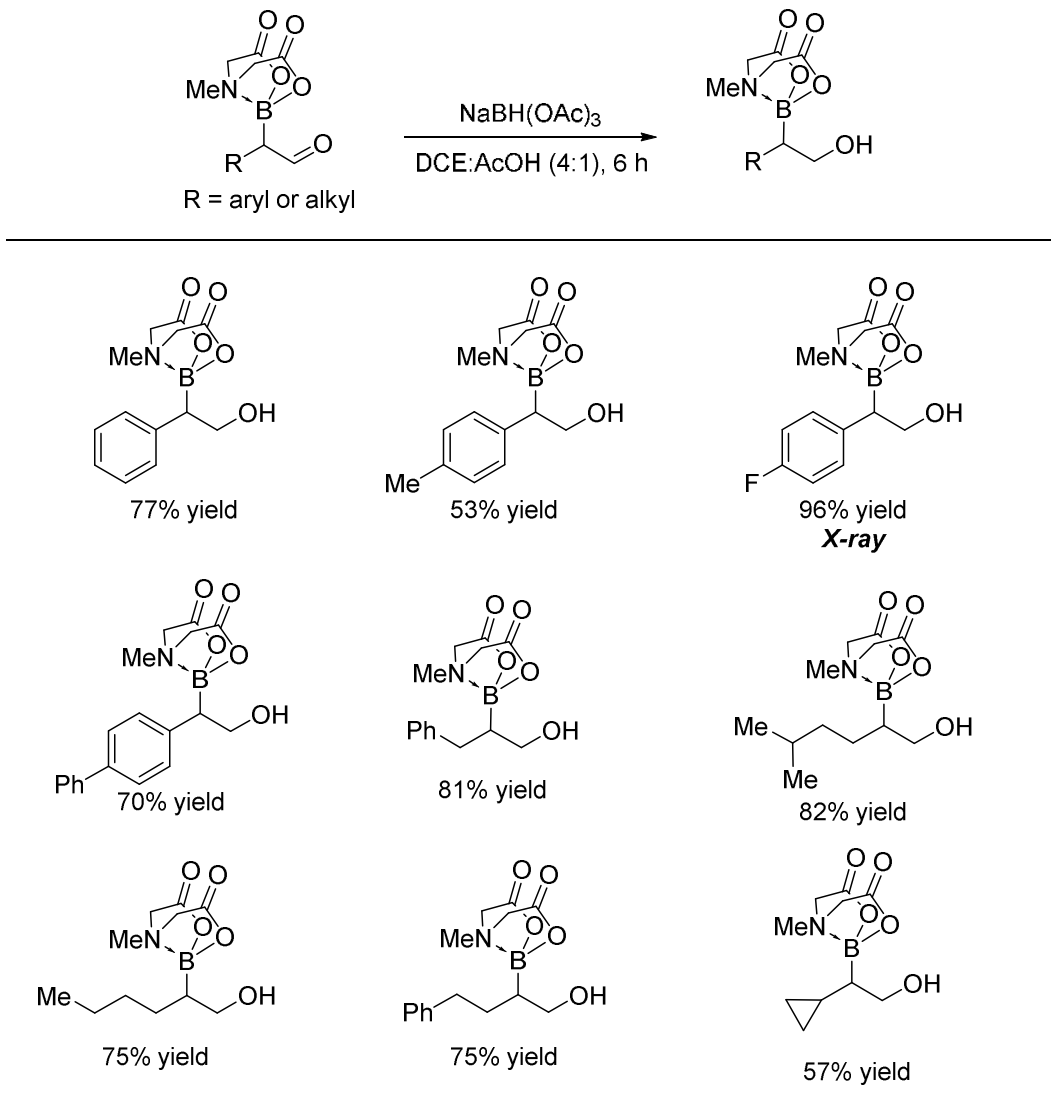

\section{General Procedure for the Synthesis of Boryl Alcohols}

To a 3-neck oven dried round bottom equipped with a magnetic stir-bar was added boryl aldehyde ( 2.0 mmol, 1.0 equiv). The flask was then immersed in an ice water bath and subsequently charged with 1,2-dichloroethane $(16 \mathrm{~mL}, 4$ vol.) and stirred for 5 minutes. AcOH (4 mL, 1 vol.) was slowly added to the reaction flask and stirred for another 10 minutes to yield a homogenous solution. The resulting molarity in boryl aldehyde was $0.1 \mathrm{M}$. $\mathrm{NaBH}(\mathrm{OAc})_{3}(1.25$ equivalents) was added to the stirring solution and allowed to stir for one hour at $0^{\circ} \mathrm{C}$. A total of 5.0 equivalents of $\mathrm{NaBH}(\mathrm{OAc})_{3}$ was added to the reaction flask at 1.25 equiv/hour over 4 hours with addition every hour. Upon complete addition of $\mathrm{NaBH}(\mathrm{OAc})_{3}$ the reaction flask was removed from the ice-water bath and allowed to warm to room temperature. Upon starting material consumption, as determined by TLC the solvent is removed in vacuo to yield an oily residue. The reaction flask was placed back into an ice-water bath and EtOAc (2 vol.) was added 
to the residue with stirring. To the cooled EtOAc solution was slowly added $\mathrm{pH} 7$ phosphate buffer and stirred for 10 minutes to destroy remaining hydride source. The mixture was then transferred to separatory funnel and extracted with EtOAc ( 5 x 2 vol.), dried and concentrated to yield a white solid. The solid was then purified by $\mathrm{SiO}_{2}$ to yield the desired alcohol.

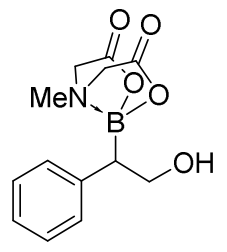

(2-hydroxy-1-phenylethyl)MIDA boronate (1a)

$0.314 \mathrm{~g}, 56 \%$ yield, white solid

${ }^{1} \mathrm{H}$ NMR $\left(400 \mathrm{MHz}\right.$, Acetonitrile- $\left.d_{3}\right)$

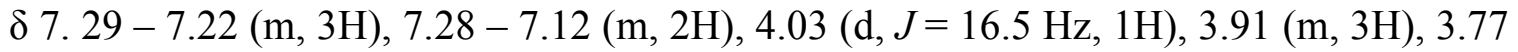

$(\mathrm{m}, 1 \mathrm{H}), 3.51(\mathrm{~d}, J=17.0 \mathrm{~Hz}, 1 \mathrm{H}), 2.99(\mathrm{~s}, 3 \mathrm{H}), 2.82(\mathrm{~m}, 1 \mathrm{H}), 2.52(\mathrm{t}, J=7.9 \mathrm{~Hz}, 1 \mathrm{H})$

${ }^{13} \mathrm{C}$ NMR $\left(126 \mathrm{MHz}\right.$, Acetonitrile- $\left.d_{3}\right)$

$\delta 169.2,169.1,143.8,130.0,129.1,126.3,65.8,63.7,63.1,47.0$

${ }^{11}$ B NMR (128 MHz, Acetonitrile- $d_{3}$ )

$\delta 12.5$

HRMS [DART] $\left(\mathrm{M}+\mathrm{NH}_{4}{ }^{+}\right)$

$m / z$ calculated for $\mathrm{C}_{13} \mathrm{H}_{20} \mathrm{BN}_{2} \mathrm{O}_{5}=295.1465$

$m / z$ found $=295.1474$

TLC (hexanes:acetone 1:2)

$$
\mathrm{R}_{\mathrm{f}}=0.31
$$

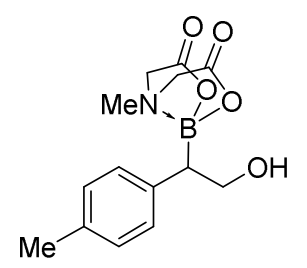

(2-hydroxy-1-(p-tolyl)ethyl)MIDA boronate (1b)

$0.308 \mathrm{~g}, 53 \%$ yield, white solid

${ }^{1} \mathrm{H}$ NMR (500 MHz, Acetonitrile- $d_{3}$ ) 
$\delta 7.15-7.13(\mathrm{~m}, 2 \mathrm{H}), 7.12-7.09(\mathrm{~m}, 2 \mathrm{H}), 4.00(\mathrm{~d}, J=16.6 \mathrm{~Hz}, 1 \mathrm{H}), 3.92-3.87(\mathrm{~m}$, 2H), $3.84(\mathrm{dd}, J=10.0,8.1 \mathrm{~Hz}, 1 \mathrm{H}), 3.73(\mathrm{dd}, J=10.0,7.8 \mathrm{~Hz}, 1 \mathrm{H}), 3.47$ (d, $J=17.0 \mathrm{~Hz}$, $1 \mathrm{H}), 2.97(\mathrm{~s}, 3 \mathrm{H}), 2.46(\mathrm{t}, J=7.9 \mathrm{~Hz}, 1 \mathrm{H}), 2.31(\mathrm{~s}, 3 \mathrm{H})$

${ }^{13} \mathrm{C}$ NMR (126 MHz, Acetonitrile- $d_{3}$ )

$\delta 168.3,168.3,139.5,134.7,128.9,128.8,64.9,62.7,62.1,45.9,20.0$

${ }^{11} \mathrm{~B}$ NMR (128 MHz, Acetonitrile- $d_{3}$ )

$\delta 12.4$

HRMS [DART-MS] $\left(\mathrm{M}+\mathrm{NH}_{4}{ }^{+}\right)$

$m / z$ calculated for $\mathrm{C}_{14} \mathrm{H}_{22} \mathrm{BN}_{2} \mathrm{O}_{5}=309.1621$

$\mathrm{m} / \mathrm{z}$ found $=309.1628$

TLC (hexanes:acetone 1:2)

$$
\mathrm{R}_{\mathrm{f}}=0.32
$$

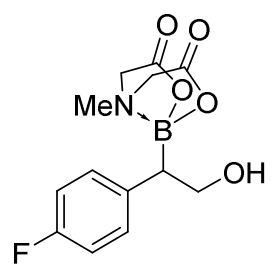

(1-(4-fluorophenyl)-2-hydroxyethyl)MIDA boronate (1c)

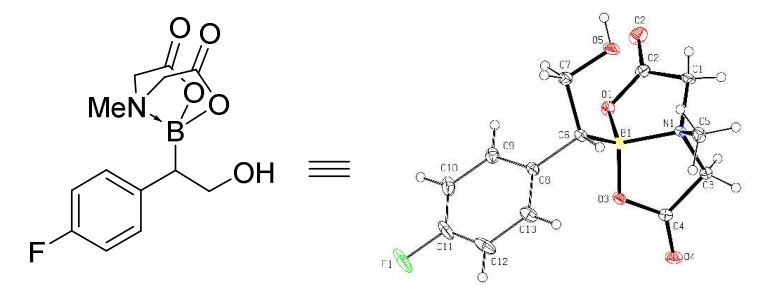

$\mathrm{X}$-ray quality crystals of $\mathbf{1 c}$ were grown from a solution of hexanes:acetone via slow evaporation $0.554 \mathrm{~g}, 94 \%$ yield, white solid

${ }^{1} \mathrm{H}$ NMR $\left(500 \mathrm{MHz}\right.$, Acetone- $d_{6}$ )

$\delta 7.31-7.25(\mathrm{~m}, 2 \mathrm{H}), 7.01-6.95(\mathrm{~m}, 2 \mathrm{H}), 4.22-4.10(\mathrm{~m}, 3 \mathrm{H}), 3.91(\mathrm{dd}, J=9.8,8.3 \mathrm{~Hz}$, $1 \mathrm{H}), 3.80-3.72(\mathrm{~m}, 2 \mathrm{H}), 3.16(\mathrm{~s}, 3 \mathrm{H}), 2.62-2.56(\mathrm{~m}, 1 \mathrm{H})$

${ }^{13} \mathrm{C}$ NMR (126 MHz, Acetone- $d_{6}$ )

$\delta$ 168.1, 167.92, $160.87(\mathrm{~d}, \mathrm{~J}=240.7 \mathrm{~Hz}), 139.13(\mathrm{~d}, \mathrm{~J}=3.2 \mathrm{~Hz}), 130.58(\mathrm{~d}, \mathrm{~J}=7.6 \mathrm{~Hz})$, $114.47(\mathrm{~d}, \mathrm{~J}=20.9 \mathrm{~Hz}) 65.1,62.8,62.1,45.8$

${ }^{11} \mathrm{~B}$ NMR (128 MHz, Acetone- $\left.d_{6}\right)$

$\delta 12.4$ 
${ }^{19}$ F NMR (282 MHz, Acetone- $\left.d_{6}\right)$

$\delta-120.39$

HRMS [DART-MS] $\left(\mathrm{M}+\mathrm{NH}_{4}{ }^{+}\right)$

$m / z$ calculated for $\mathrm{C}_{13} \mathrm{H}_{19} \mathrm{BFN}_{2} \mathrm{O}_{5}=313.1370$

$\mathrm{m} / \mathrm{z}$ found $=313.1370$

TLC (hexanes:acetone 1:2)

$$
\mathrm{R}_{\mathrm{f}}=0.34
$$

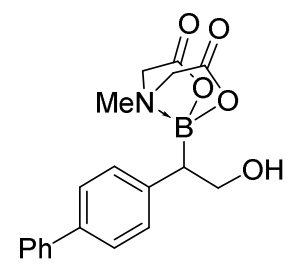

(1-([1,1'-biphenyl]-4-yl)-2-hydroxyethyl)MIDA boronate (1d)

$0.451 \mathrm{~g}, 64 \%$ yield, white solid

${ }^{1} \mathrm{H}$ NMR (400 MHz, Acetone- $d_{6}$ )

$\delta 7.69-7.64(\mathrm{~m}, 2 \mathrm{H}), 7.59-7.53(\mathrm{~m}, 2 \mathrm{H}), 7.46(\mathrm{dd}, J=8.4,7.0 \mathrm{~Hz}, 2 \mathrm{H}), 7.42-7.38(\mathrm{~m}$, $2 \mathrm{H}), 7.37-7.31(\mathrm{~m}, 1 \mathrm{H}), 4.32-4.10(\mathrm{~m}, 3 \mathrm{H}), 4.01(\mathrm{~m}, 1 \mathrm{H}), 3.87(\mathrm{~m}, 1 \mathrm{H}), 3.76(\mathrm{~d}, J=$ $17.0 \mathrm{~Hz}, 1 \mathrm{H}), 3.22(\mathrm{~s}, 3 \mathrm{H}), 2.69-2.61(\mathrm{~m}, 1 \mathrm{H})$

${ }^{13} \mathrm{C}$ NMR $\left(101 \mathrm{MHz}\right.$, Acetone- $\left.d_{6}\right)$

$\delta 169.0,168.8,143.4,141.9,138.6,130.5,129.6,127.7,127.4,127.3,66.0,63.7,63.0$, 46.8

${ }^{11} \mathrm{~B}$ NMR (128 MHz, Acetone- $\left.d_{6}\right)$

$\delta 12.5$

HRMS [DART-MS] $\left(\mathrm{M}+\mathrm{NH}_{4}^{+}\right)$

$m / z$ calculated for $\mathrm{C}_{19} \mathrm{H}_{24} \mathrm{BN}_{2} \mathrm{O}_{5}=371.1772$

$\mathrm{m} / \mathrm{z}$ found $=371.1770$

TLC (hexanes:acetone 3:4)

$$
\mathrm{R}_{\mathrm{f}}=0.28
$$

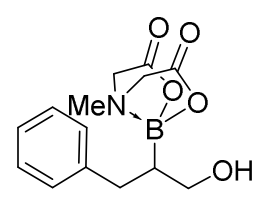

(1-hydroxy-3-phenylpropan-2-yl)MIDA boronate (1e) 
$0.471 \mathrm{~g}, 81 \%$ yield, white solid

${ }^{1} \mathrm{H}$ NMR $\left(500 \mathrm{MHz}\right.$, Acetone- $\left.d_{6}\right)$

$\delta 7.33-7.17(\mathrm{~m}, 4 \mathrm{H}), 7.18-7.10(\mathrm{~m}, 1 \mathrm{H}), 4.22(\mathrm{~m}, 2 \mathrm{H}), 4.11-4.02(\mathrm{~m}, 2 \mathrm{H}), 3.91-$ $3.83(\mathrm{~m}, 1 \mathrm{H}), 3.49(\mathrm{~m}, 1 \mathrm{H}), 3.42(\mathrm{~m}, 1 \mathrm{H}), 3.26(\mathrm{~s}, 3 \mathrm{H}), 3.00(\mathrm{dd}, J=14.0,3.4 \mathrm{~Hz}, 1 \mathrm{H})$, $2.40(\mathrm{dd}, J=14.0,11.6 \mathrm{~Hz}, 1 \mathrm{H}), 1.52(\mathrm{~m}, 1 \mathrm{H})$

${ }^{13} \mathrm{C}$ NMR (126 MHz, Acetone- $\left.d_{6}\right)$

$\delta 168.3,168.1,143.1,128.6,128.0,125.3,62.9,62.4,62.1,45.7,33.8$

${ }^{11} \mathrm{~B}$ NMR (128 MHz, Acetone- $d_{6}$ )

$\delta 13.4$

IR (thin film, $\mathrm{NaCl}$ )

$v 3207,3024,2359,2341,2332,1747,1624,1452,1342,1300,1251,1193,1159,1105$, $1070,1026,962,893,734,700$

HRMS [DART-MS] $\left(\mathrm{M}+\mathrm{NH}_{4}{ }^{+}\right)$

$m / z$ calculated for $\mathrm{C}_{14} \mathrm{H}_{22} \mathrm{BN}_{2} \mathrm{O}_{5}=309.1621$

$\mathrm{m} / \mathrm{z}$ found $=309.1628$

TLC (hexanes:acetone 1:1)

$$
\mathrm{R}_{\mathrm{f}}=0.28
$$

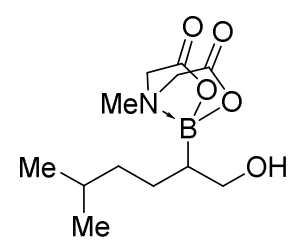

(1-hydroxy-5-methylhexan-2-yl)MIDA boronate (1f)

$0.444 \mathrm{~g}, 82 \%$ yield, white solid

${ }^{1} \mathrm{H}$ NMR (400 MHz, Acetonitrile- $d_{3}$ )

$\delta 4.04(\mathrm{~d}, J=16.4 \mathrm{~Hz}, 1 \mathrm{H}), 3.95(\mathrm{~d}, J=17.2 \mathrm{~Hz}, 1 \mathrm{H}), 3.88(\mathrm{~d}, J=8.1 \mathrm{~Hz}, 1 \mathrm{H}), 3.84(\mathrm{~d}, J$ $=7.3 \mathrm{~Hz}, 1 \mathrm{H}), 3.64(\mathrm{dd}, J=9.7,5.3 \mathrm{~Hz}, 1 \mathrm{H}), 3.47(\mathrm{t}, J=9.3 \mathrm{~Hz}, 1 \mathrm{H}), 3.01(\mathrm{~s}, 3 \mathrm{H}), 1.58-$ $1.42(\mathrm{~m}, 2 \mathrm{H}), 1.36-1.17(\mathrm{~m}, 3 \mathrm{H}), 1.13-1.02(\mathrm{~m}, 1 \mathrm{H}), 0.91(\mathrm{dd}, J=6.6,3.4 \mathrm{~Hz}, 6 \mathrm{H})$

${ }^{13} \mathrm{C}$ NMR (101 MHz, Acetonitrile- $d_{3}$ )

$\delta 169.7,169.3,63.8,63.5,62.9,46.7,38.8,29.1,26.3,22.9,22.5$

${ }^{11}$ B NMR (128 MHz, Acetonitrile- $d_{3}$ ) 
$\delta 13.2$

HRMS [DART-MS] $\left(\mathrm{M}+\mathrm{NH}_{4}{ }^{+}\right)$

$m / z$ calculated for $\mathrm{C}_{12} \mathrm{H}_{26} \mathrm{BN}_{2} \mathrm{O}_{5}=289.1934$

$\mathrm{m} / \mathrm{z}$ found $=289.1942$

TLC (hexanes:acetone 1:1)

$$
\mathrm{R}_{\mathrm{f}}=0.25
$$

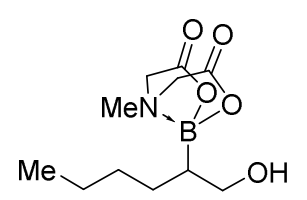

(1-hydroxyhexan-2-yl)MIDA boronate (1g)

$0.385 \mathrm{~g}, 75 \%$ yield, white solid

${ }^{1} \mathrm{H}$ NMR (400 MHz, Acetone- $d_{6}$ )

$\delta 4.21(\mathrm{~d}, J=7.7 \mathrm{~Hz}, 1 \mathrm{H}), 4.16(\mathrm{~d}, J=8.7 \mathrm{~Hz}, 1 \mathrm{H}), 4.05(\mathrm{~d}, J=6.2 \mathrm{~Hz}, 1 \mathrm{H}), 4.01(\mathrm{~d}, J=$

$5.2 \mathrm{~Hz}, 1 \mathrm{H}), 3.72(\mathrm{dd}, J=9.4,5.0 \mathrm{~Hz}, 1 \mathrm{H}), 3.54(\mathrm{t}, J=9.4 \mathrm{~Hz}, 1 \mathrm{H}), 3.22(\mathrm{~s}, 3 \mathrm{H}), 1.55$

$(\mathrm{m}, 1 \mathrm{H}), 1.43(\mathrm{~m}, 1 \mathrm{H}), 1.38-1.23(\mathrm{~m}, 4 \mathrm{H}), 1.23-1.09(\mathrm{~m}, 1 \mathrm{H}), 0.91(\mathrm{t}, J=6.9 \mathrm{~Hz}, 3 \mathrm{H})$

${ }^{13} \mathrm{C}$ NMR (101 MHz, Acetone- $\left.d_{6}\right)$

$\delta 169.5,169.0,64.1,63.7,63.0,46.6,32.1,28.6,23.9,14.4$

${ }^{11} \mathrm{~B}$ NMR (128 MHz, Acetone- $\left.d_{6}\right)$

$\delta 13.3$

HRMS [ESI-MS] $\left(\mathrm{M}+\mathrm{Na}^{+}\right)$

$m / z$ calculated for $\mathrm{C}_{11} \mathrm{H}_{20} \mathrm{BNO}_{5} \mathrm{Na}=280.1326$

$\mathrm{m} / \mathrm{z}$ found $=280.1317$

TLC (hexanes:acetone 1:1)

$$
\mathrm{R}_{\mathrm{f}}=0.27
$$

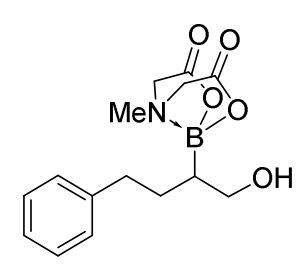

(1-hydroxy-4-phenylbutan-2-yl)MIDA boronate (1h)

$0.408 \mathrm{~g}, 67 \%$ yield, white solid

${ }^{1} \mathrm{H}$ NMR (399 MHz, Acetonitrile- $d_{3}+\mathrm{D}_{2} \mathrm{O}$ ) 
$\delta 7.33-7.26(\mathrm{~m}, 2 \mathrm{H}), 7.26-7.22(\mathrm{~m}, 2 \mathrm{H}), 7.21-7.16(\mathrm{~m}, 1 \mathrm{H}), 4.01(\mathrm{~d}, J=16.4 \mathrm{~Hz}$, $1 \mathrm{H}), 3.94(\mathrm{~d}, J=17.1 \mathrm{~Hz}, 1 \mathrm{H}), 3.87(\mathrm{~d}, J=7.9 \mathrm{~Hz}, 1 \mathrm{H}), 3.83(\mathrm{~d}, J=7.3 \mathrm{~Hz}, 1 \mathrm{H}), 3.70$ (m, 1H), 3.55 (td, $J=9.3,3.7 \mathrm{~Hz}, 1 \mathrm{H}), 2.97(\mathrm{~s}, 3 \mathrm{H}), 2.86(\mathrm{t}, J=3.8 \mathrm{~Hz}, 1 \mathrm{H}), 2.74(\mathrm{~m}$, $1 \mathrm{H}), 2.60(\mathrm{~m}, 1 \mathrm{H}), 1.82-1.70(\mathrm{~m}, 1 \mathrm{H}), 1.57(\mathrm{~m}, 1 \mathrm{H}), 1.16(\mathrm{~m}, 1 \mathrm{H})$

${ }^{13} \mathrm{C}$ NMR $\left(126 \mathrm{MHz}\right.$, Acetonitrile- $\left.d_{3}+\mathrm{D}_{2} \mathrm{O}\right)$

$\delta 168.7,168.4,143.3,128.3,128.2,125.5,62.8,62.7,62.2,45.9,34.6,29.9$

HRMS [DART-MS] $\left(\mathrm{M}+\mathrm{NH}_{4}{ }^{+}\right)$

$m / z$ calculated for $\mathrm{C}_{15} \mathrm{H}_{24} \mathrm{BN}_{2} \mathrm{O}_{5}=323.1778$

$m / z$ found $=323.1776$

TLC (hexanes:acetone 1:2)

$$
\mathrm{R}_{\mathrm{f}}=0.25
$$

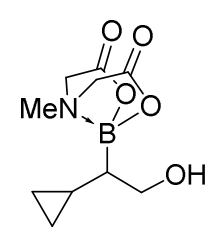

(1-cyclopropyl-2-hydroxyethyl)MIDA boronate (1i)

$0.274 \mathrm{~g}, 57 \%$ yield, white solid

${ }^{1} \mathrm{H}$ NMR (400 MHz, Acetonitrile- $d_{3}$ )

$\delta 4.01-3.74(\mathrm{~m}, 4 \mathrm{H}), 3.63(\mathrm{dd}, J=9.7,5.3 \mathrm{~Hz}, 1 \mathrm{H}), 3.48(\mathrm{t}, J=9.2 \mathrm{~Hz}, 1 \mathrm{H}), 2.93(\mathrm{~s}$, $3 \mathrm{H}), 2.76(\mathrm{br} \mathrm{s}, 1 \mathrm{H}), 0.54(\mathrm{~m}, 1 \mathrm{H}), 0.48-0.39(\mathrm{~m}, 1 \mathrm{H}), 0.37-0.23(\mathrm{~m}, 2 \mathrm{H}), 0.13(\mathrm{~m}$, $1 \mathrm{H}),-0.01(\mathrm{~m}, 1 \mathrm{H})$.

${ }^{13} \mathrm{C}$ NMR (101 MHz, Acetonitrile- $d_{3}$ )

$\delta 168.5,168.1,63.5,62.2,61.8,45.6,29.5,9.9,4.5,3.5$

${ }^{11}$ B NMR (128 MHz, Acetonitrile- $\left.d_{3}\right)$

$\delta 13.2$

HRMS [DART-MS] $\left(\mathrm{M}+\mathrm{NH}_{4}{ }^{+}\right)$

$m / z$ calculated for $\mathrm{C}_{10} \mathrm{H}_{20} \mathrm{BN}_{2} \mathrm{O}_{5}=259.1465$

$m / z$ found $=259.1461$

TLC (hexanes:acetone 1:2)

$$
\mathrm{R}_{\mathrm{f}}=0.41
$$




\section{General Procedure for the Synthesis of $\alpha$-Boryl Sulfamate Esters}

To an oven dried three-neck round bottom flask equipped with a magnetic stir bar was added $\mathrm{ClSO}_{2} \mathrm{NCO}$ (1.5 equiv.) The reaction flask was then immersed in an ice-water bath and stirred for 10 minutes at which time $99 \%$ formic acid (1.5 equiv.) was added to the mixture dropwise over 5 minutes. The solution solidified within that time. The flask was allowed to age for 5 minutes then $\mathrm{MeCN}(4 \mathrm{~mL}$ ) was added along the wall and allowed to stir overnight (12 hours) slowly warming to room temperature. The flask was then cooled back to $0{ }^{\circ} \mathrm{C}$ and a solution of boryl alcohol (3.0 mmol, 1.0 equiv) pyridine (1.5 equiv.) and $\mathrm{MeCN}(4 \mathrm{~mL})$ was added via syringe pump over the course of 30 minutes. The syringe pump was removed following the addition and the reaction mixture was removed from the ice bath and allowed to warm to room temperature. Upon complete consumption of alcohol (2-5 hours) EtOAc and $\mathrm{H}_{2} \mathrm{O}$ were added to the reaction flask and subsequently transferred to a separatory funnel. The aqueous layer was extracted $5 \mathrm{x}$ and then the combined organics were dried with $\mathrm{Na}_{2} \mathrm{SO}_{4}$, filtered and concentrated to yield a clear oily residue which was purified via $\mathrm{SiO}_{2}$ to provide the desired sulfamate esters as white solids.

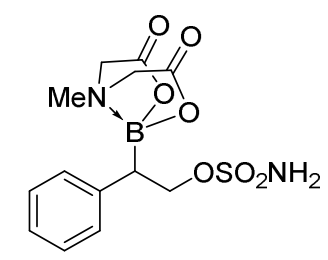

(1-phenyl-2-(sulfamoyloxy)ethyl)MIDA boronate (2a)

$0.758 \mathrm{~g}, 71 \%$ yield, white solid

${ }^{1} \mathrm{H}$ NMR (400 MHz, Acetonitrile- $\left.d_{3}\right)$

$\delta 7.39-7.30(\mathrm{~m}, 4 \mathrm{H}), 7.26(\mathrm{~m}, 1 \mathrm{H}), 5.60(\mathrm{~s}, 2 \mathrm{H}), 4.56(\mathrm{dd}, J=9.8,5.1 \mathrm{~Hz}, 1 \mathrm{H}), 4.46(\mathrm{dd}$, $J=10.7,9.8 \mathrm{~Hz}, 1 \mathrm{H}), 4.00(\mathrm{~d}, J=17.1 \mathrm{~Hz}, 1 \mathrm{H}), 3.95-3.83(\mathrm{~m}, 2 \mathrm{H}), 3.23(\mathrm{~d}, J=16.9$ $\mathrm{Hz}, 1 \mathrm{H}), 2.90(\mathrm{~s}, 3 \mathrm{H}), 2.85-2.76(\mathrm{~m}, 1 \mathrm{H})$

${ }^{13} \mathrm{C}$ NMR (101 MHz, Acetonitrile- $d_{3}$ )

$\delta$ 167.6, 167.1, 140.0, 128.6, 128.2, 125.7, 72.9, 62.1, 62.0, 45.6, 29.5

${ }^{11}$ B NMR $\left(128 \mathrm{MHz}\right.$, , Acetonitrile- $\left.d_{3}\right)$

$\delta 11.5$

HRMS [DART-MS] $\left(\mathrm{M}+\mathrm{H}^{+}\right)$

$m / z$ calculated for $\mathrm{C}_{13} \mathrm{H}_{18} \mathrm{BN}_{2} \mathrm{O}_{7} \mathrm{~S}=357.0927$ 
$m / z$ found $=357.0925$

TLC (hexanes:acetone 1:2)

$$
\mathrm{R}_{\mathrm{f}}=0.39
$$

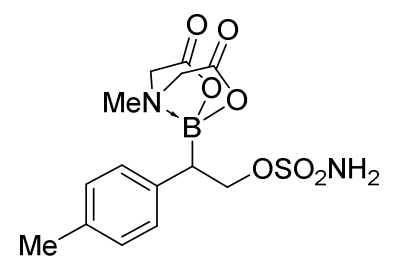

(2-(sulfamoyloxy)-1-(p-tolyl)ethyl)MIDA boronate (2b)

$0.588 \mathrm{~g}, 53 \%$ yield, hygroscopic white solid

${ }^{1} \mathrm{H}$ NMR (400 MHz, Acetonitrile- $\left.d_{3}\right)$

$\delta 7.23-7.16(\mathrm{~m}, 4 \mathrm{H}), 5.56(\mathrm{br} \mathrm{s}, 2 \mathrm{H}), 4.50(\mathrm{dd}, J=9.8,5.0 \mathrm{~Hz}, 1 \mathrm{H}), 4.39(\mathrm{dd}, J=10.8$,

$9.8 \mathrm{~Hz}, 1 \mathrm{H}), 3.95(\mathrm{~d}, J=17.2 \mathrm{~Hz}, 1 \mathrm{H}), 3.91-3.78(\mathrm{~m}, 2 \mathrm{H}), 3.16(\mathrm{~d}, J=16.9 \mathrm{~Hz}, 1 \mathrm{H})$, $2.86(\mathrm{~s}, 3 \mathrm{H}), 2.71(\mathrm{dd}, J=10.7,5.0 \mathrm{~Hz}, 1 \mathrm{H}), 2.30(\mathrm{~s}, 3 \mathrm{H})$

${ }^{13} \mathrm{C}$ NMR (101 MHz, Acetonitrile- $\left.d_{3}\right)$

$\delta 167.8,167.3,136.9,135.6,129.1,128.7,73.2,62.3,62.2,45.8,29.7,19.9$

${ }^{11} \mathrm{~B}$ NMR (128 MHz, Acetonitrile- $d_{3}$ )

$\delta 11.5$

HRMS [DART-MS] $\left(\mathrm{M}+\mathrm{H}^{+}\right)$

$m / z$ calculated for $\mathrm{C}_{14} \mathrm{H}_{20} \mathrm{BN}_{2} \mathrm{O}_{7} \mathrm{~S}=371.1084$

$\mathrm{m} / \mathrm{z}$ found $=371.1087$

TLC (EtOAc:MeCN 9:1)

$$
\mathrm{R}_{\mathrm{f}}=0.25
$$

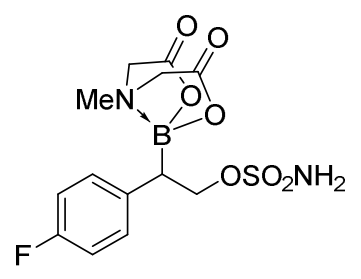

(1-(4-fluorophenyl)-2-(sulfamoyloxy)ethyl)MIDA boronate (2c)

$0.482 \mathrm{~g}, 43 \%$ yield, white solid

${ }^{1} \mathrm{H}$ NMR (500 MHz, Acetone- $d_{6}$ ) 
$\delta 7.40-7.24$ (m, 2H), $7.04-7.00(\mathrm{~m}, 2 \mathrm{H}), 4.54$ (dd, $J=9.9,5.0 \mathrm{~Hz}, 1 \mathrm{H}), 4.39$ (dd, $J=$ $10.7,9.8 \mathrm{~Hz}, 1 \mathrm{H}), 4.27(\mathrm{~d}, J=17.1 \mathrm{~Hz}, 1 \mathrm{H}), 4.18(\mathrm{~d}, J=17.0 \mathrm{~Hz}, 1 \mathrm{H}), 4.11(\mathrm{~d}, J=17.1$ $\mathrm{Hz}, 1 \mathrm{H}), 3.51(\mathrm{~d}, J=17.0 \mathrm{~Hz}, 1 \mathrm{H}), 3.08(\mathrm{~s}, 3 \mathrm{H}), 2.84(\mathrm{dd}, J=10.7,5.0 \mathrm{~Hz}, 1 \mathrm{H})$

${ }^{13} \mathrm{C}$ NMR (101 MHz, Acetone- $\left.d_{6}\right)$

$\delta 167.3,167.0,161.0(\mathrm{~d}, J=242.0 \mathrm{~Hz}), 136.5(\mathrm{~d}, J=3.3 \mathrm{~Hz}), 130.4$ (d, $J=7.8 \mathrm{~Hz}), 114.7$ (d, $J=21.0 \mathrm{~Hz}), 72.6,62.3,62.2,45.7$

${ }^{11} \mathrm{~B}$ NMR $\left(128 \mathrm{MHz}\right.$, Acetone- $\left.d_{6}\right)$

$\delta 11.5$

${ }^{19}$ F NMR $\left(282 \mathrm{MHz}\right.$, Acetone- $\left.d_{6}\right)$

$\delta-119.1$

IR (thin film, $\mathrm{NaCl}$ )

v 2956, 2922, 2854, 2343, 2324, 1766, 1747, 1508, 1363, 1340, 1301, 1178, 1028

HRMS [DART-MS] $\left(\mathrm{M}+\mathrm{Na}^{+}\right)$

$m / z$ calculated for $\mathrm{C}_{13} \mathrm{H}_{16} \mathrm{BN}_{2} \mathrm{O}_{7} \mathrm{FNaS}=397.0658$

$m / z$ found $=397.0648$

TLC (hexanes:acetone 3:4)

$$
\mathrm{R}_{\mathrm{f}}=0.23
$$

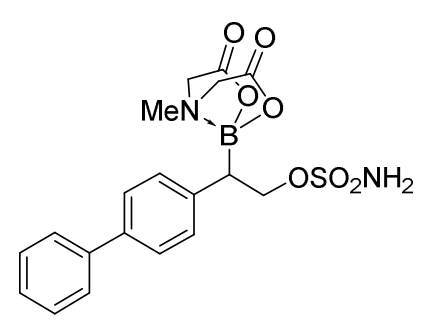

(1-([1,1'-biphenyl]-4-yl)-2-(sulfamoyloxy)ethyl)MIDA boronate (2d)

$0.518 \mathrm{~g}, 40 \%$ yield, white solid

${ }^{1} \mathrm{H}$ NMR (500 MHz, Acetonitrile- $d_{3}$ )

$\delta 7.70-7.66(\mathrm{~m}, 2 \mathrm{H}), 7.65-7.61(\mathrm{~m}, 2 \mathrm{H}), 7.51-7.44(\mathrm{~m}, 2 \mathrm{H}), 7.43-7.34(\mathrm{~m}, 3 \mathrm{H})$, $5.63(\mathrm{~s}, 2 \mathrm{H}), 4.57(\mathrm{dd}, J=9.9,5.1 \mathrm{~Hz}, 1 \mathrm{H}), 4.48(\mathrm{dd}, J=10.8,9.9 \mathrm{~Hz}, 1 \mathrm{H}), 4.01(\mathrm{~d}, J=$ $17.1 \mathrm{~Hz}, 1 \mathrm{H}), 3.96-3.86(\mathrm{~m}, 2 \mathrm{H}), 3.33(\mathrm{~d}, J=16.9 \mathrm{~Hz}, 1 \mathrm{H}), 2.93(\mathrm{~s}, 3 \mathrm{H}), 2.85$ (dd, $J=$ $10.7,5.1 \mathrm{~Hz}, 1 \mathrm{H})$

${ }^{13} \mathrm{C}$ NMR $\left(126 \mathrm{MHz}\right.$, Acetonitrile- $\left.d_{3}\right)$

$\delta 167.9,167.4,140.5,139.7,138.6,129.5,128.8,128.8,127.2,127.0,126.7,126.6,73.1$, $62.5,62.4,46.0$ 
${ }^{11} \mathrm{~B}$ NMR (128 MHz, Acetonitrile- $\left.d_{3}\right)$

$\delta 11.6$

HRMS [DART-MS] $\left(\mathrm{M}+\mathrm{H}^{+}\right)$

$m / z$ calculated for $\mathrm{C}_{19} \mathrm{H}_{22} \mathrm{BN}_{2} \mathrm{O}_{7} \mathrm{~S}=433.1240$

$\mathrm{m} / \mathrm{z}$ found $=433.1249$

TLC (hexanes:acetone 1:2)

$$
\mathrm{R}_{\mathrm{f}}=0.46
$$

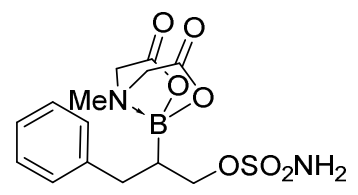

(1-phenyl-3-(sulfamoyloxy)propan-2-yl)MIDA boronate (2e)

$0.666 \mathrm{~g}, 60 \%$ yield, white solid

${ }^{1} \mathrm{H}$ NMR (500 MHz, Acetone- $\left.d_{6}\right)$

$\delta 7.33-7.24(\mathrm{~m}, 4 \mathrm{H}), 7.24-7.14(\mathrm{~m}, 1 \mathrm{H}), 4.29(\mathrm{~d}, J=17.2 \mathrm{~Hz}, 1 \mathrm{H}), 4.23(\mathrm{~d}, J=16.9$

$\mathrm{Hz}, 1 \mathrm{H}), 4.09-3.99(\mathrm{~m}, 4 \mathrm{H}), 3.21(\mathrm{~s}, 3 \mathrm{H}), 3.02-2.97(\mathrm{~m}, 1 \mathrm{H}), 2.67-2.58(\mathrm{~m}, 1 \mathrm{H}), 1.90$

$-1.69(\mathrm{~m}, 1 \mathrm{H})$

${ }^{13} \mathrm{C}$ NMR $\left(126 \mathrm{MHz}\right.$, Acetone- $\left.d_{6}\right)$

$\delta 167.8,167.8,141.7,128.8,128.2,125.8,70.9,62.9,62.5,46.1,33.4$

${ }^{11} \mathrm{~B}$ NMR $\left(128 \mathrm{MHz}\right.$, Acetone- $\left.d_{6}\right)$

$\delta 12.6$

HRMS [DART-MS] $\left(\mathrm{M}+\mathrm{NH}_{4}^{+}\right)$

$m / z$ calculated for $\mathrm{C}_{14} \mathrm{H}_{23} \mathrm{BN}_{3} \mathrm{O}_{7} \mathrm{~S}=388.1344$

$\mathrm{m} / \mathrm{z}$ found $=388.1330$

TLC (DCM:acetone 3:1)

$$
\mathrm{R}_{\mathrm{f}}=0.52
$$

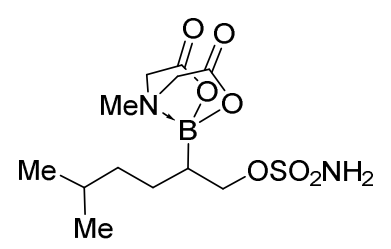

(5-methyl-1-(sulfamoyloxy)hexan-2-yl)MIDA boronate (2f) 
$0.388 \mathrm{~g}, 37 \%$ yield, white solid

${ }^{1} \mathrm{H}$ NMR $\left(400 \mathrm{MHz}\right.$, Acetonitrile- $\left.d_{3}\right)$

$\delta 5.66(\mathrm{br} \mathrm{s}, 2 \mathrm{H}), 4.16-4.12(\mathrm{~m}, 2 \mathrm{H}), 3.99(\mathrm{~d}, J=9.0 \mathrm{~Hz}, 1 \mathrm{H}), 3.95(\mathrm{~d}, J=9.0 \mathrm{~Hz}, 1 \mathrm{H})$, $3.86(\mathrm{~d}, J=6.5 \mathrm{~Hz}, 1 \mathrm{H}), 3.82(\mathrm{~d}, J=6.5 \mathrm{~Hz}, 1 \mathrm{H}), 2.95(\mathrm{~s}, 3 \mathrm{H}), 1.60-1.44(\mathrm{~m}, 2 \mathrm{H}), 1.44$ -1.19 (m, 4H), 0.89 (dd, $J=6.6,3.3 \mathrm{~Hz}, 6 \mathrm{H})$

${ }^{13} \mathrm{C}$ NMR $\left(100 \mathrm{MHz}\right.$, Acetonitrile- $\left.d_{3}\right)$

$\delta 168.1,72.1,62.7,62.4,46.1,37.3,28.2,25.0,22.1,21.7$

${ }^{11}$ B NMR (96 MHz, Acetonitrile- $d_{3}$ )

$\delta 12.6$

HRMS [DART-MS] $\left(\mathrm{M}+\mathrm{Na}^{+}\right)$

$m / z$ calculated for $\mathrm{C}_{12} \mathrm{H}_{23} \mathrm{BN}_{2} \mathrm{O}_{7} \mathrm{SNa}=372.1248$

$\mathrm{m} / \mathrm{z}$ found $=372.1249$

TLC (EtOAc:MeCN 9:1)

$$
\mathrm{R}_{\mathrm{f}}=0.54
$$

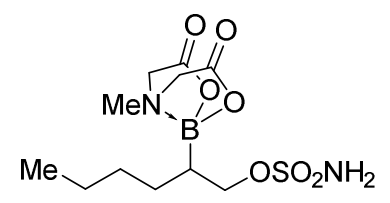

(1-(sulfamoyloxy)hexan-2-yl)MIDA boronate (2g)

$0.412 \mathrm{~g}, 41 \%$ yield, white solid

${ }^{1} \mathrm{H}$ NMR (400 MHz, Acetone- $d_{6}$ )

$\delta 6.64(\mathrm{~s}, 2 \mathrm{H}), 4.32-4.20(\mathrm{~m}, 4 \mathrm{H}), 4.09(\mathrm{~m}, 2 \mathrm{H}), 3.23(\mathrm{~s}, 3 \mathrm{H}), 1.69-1.57(\mathrm{~m}, 1 \mathrm{H}), 1.55$

-1.39 (m, 3H), $1.39-1.25$ (m, 3H), $0.92(\mathrm{t}, J=7.2 \mathrm{~Hz}, 3 \mathrm{H})$

${ }^{13} \mathrm{C}$ NMR $\left(101 \mathrm{MHz}\right.$, Acetone- $\left.d_{6}\right)$

$\delta 168.7,168.7,72.7,63.7,63.3,46.8,31.4,28.4,23.8,14.3$

${ }^{11} \mathrm{~B}$ NMR $\left(128 \mathrm{MHz}\right.$, Acetone- $\left.d_{6}\right)$

$\delta 12.5$

HRMS [ESI-MS] $\left(\mathrm{M}+\mathrm{Na}^{+}\right)$

$m / z$ calculated for $\mathrm{C}_{11} \mathrm{H}_{21} \mathrm{BN}_{2} \mathrm{NaO}_{7} \mathrm{~S}=359.1089$ 
$m / z$ found $=359.1089$

TLC (hexanes:acetone 3:4)

$$
\mathrm{R}_{\mathrm{f}}=0.37
$$

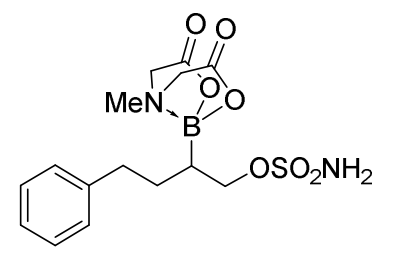

(4-phenyl-1-(sulfamoyloxy)butan-2-yl)MIDA boronate (2h)

$0.633 \mathrm{~g}, 55 \%$ yield, white solid

${ }^{1} \mathrm{H}$ NMR (500 MHz, Acetonitrile- $\left.d_{3}\right)$

$\delta 7.33-7.18(\mathrm{~m}, 5 \mathrm{H}), 5.68(\mathrm{~s}, 2 \mathrm{H}), 4.31-4.22(\mathrm{~m}, 2 \mathrm{H}), 3.98(\mathrm{~m}, 2 \mathrm{H}), 3.89-3.81(\mathrm{~m}$, 2H), $2.89(\mathrm{~s}, 3 \mathrm{H}), 2.84-2.78(\mathrm{~m}, 1 \mathrm{H}), 2.68-2.60(\mathrm{~m}, 1 \mathrm{H}), 1.86-1.65(\mathrm{~m}, 2 \mathrm{H}), 1.44-$ $1.39(\mathrm{~m}, 1 \mathrm{H})$

${ }^{13} \mathrm{C}$ NMR (126 MHz, Acetonitrile- $\left.d_{3}\right)$

$\delta 167.9(2 \mathrm{C}), 142.7,128.4,128.3,125.7,71.9,62.6,62.4,46.0,34.0,29.5$

${ }^{11} \mathrm{~B}$ NMR (128 MHz, Acetonitrile- $\left.d_{3}\right)$

$\delta 12.3$

HRMS [DART-MS] $\left(\mathrm{M}+\mathrm{H}^{+}\right)$

$m / z$ calculated for $\mathrm{C}_{15} \mathrm{H}_{22} \mathrm{BN}_{2} \mathrm{O}_{7} \mathrm{~S}=385.1240$

$\mathrm{m} / \mathrm{z}$ found $=385.1250$

TLC (hexanes:acetone 1:2)

$\mathrm{R}_{\mathrm{f}}=0.59$

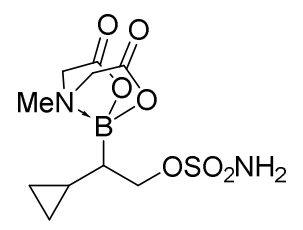

(1-cyclopropyl-2-(sulfamoyloxy)ethyl)MIDA boronate (2i)

$0.537 \mathrm{~g}, 56 \%$ yield, white solid

${ }^{1} \mathrm{H}$ NMR (500 MHz, Acetonitrile- $\left.d_{3}\right)$

$\delta 5.67(\mathrm{~s}, 2 \mathrm{H}), 4.24-4.18(\mathrm{~m}, 2 \mathrm{H}), 4.00(\mathrm{dd}, J=17.0,9.3 \mathrm{~Hz}, 2 \mathrm{H}), 3.86(\mathrm{dd}, J=17.0$, $5.0 \mathrm{~Hz}, 2 \mathrm{H}), 2.96(\mathrm{~s}, 3 \mathrm{H}), 0.73-0.60(\mathrm{~m}, 2 \mathrm{H}), 0.57-0.44(\mathrm{~m}, 2 \mathrm{H}), 0.22-0.19(\mathrm{~m}, 2 \mathrm{H})$ 
${ }^{13} \mathrm{C}$ NMR $\left(101 \mathrm{MHz}\right.$, Acetonitrile- $\left.d_{3}\right)$

$\delta 168.0,168.0,73.0,62.4,62.3,46.1,9.8,4.8,3.8$

${ }^{11}$ B NMR (128 MHz, Acetonitrile- $\left.d_{3}\right)$

$\delta 12.4$

HRMS [DART-MS] $\left(\mathrm{M}+\mathrm{H}^{+}\right)$

$m / z$ calculated for $\mathrm{C}_{10} \mathrm{H}_{18} \mathrm{BN}_{2} \mathrm{O}_{7} \mathrm{~S}=321.0927$

$\mathrm{m} / \mathrm{z}$ found $=321.0940$

TLC (hexanes:acetone 1:2)

$$
\mathrm{R}_{\mathrm{f}}=0.31
$$

\section{Characterization Data for $\alpha$-Boryl Formate Esters}

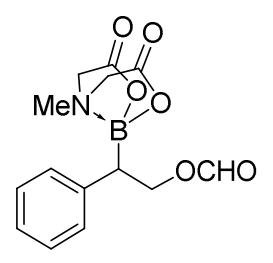

(2-(formyloxy)-1-phenylethyl)MIDA boronate (2a-formate)

$0.247 \mathrm{~g}, 27 \%$ yield, white solid

${ }^{1} \mathrm{H}$ NMR (400 MHz, Acetonitrile- $d_{3}$ )

$\delta 7.96(\mathrm{~s}, 1 \mathrm{H}), 7.42-7.31(\mathrm{~m}, 4 \mathrm{H}), 7.31(\mathrm{~s}, 1 \mathrm{H}), 4.66(\mathrm{dd}, J=11.2,4.6 \mathrm{~Hz}, 1 \mathrm{H}), 4.45(\mathrm{~d}$, $J=11.1 \mathrm{~Hz}, 1 \mathrm{H}), 3.99$ (d, $J=17.2 \mathrm{~Hz}, 1 \mathrm{H}), 3.90(\mathrm{~d}, J=5.1 \mathrm{~Hz}, 1 \mathrm{H}), 3.86(\mathrm{~d}, J=5.4 \mathrm{~Hz}$, $1 \mathrm{H}), 3.24$ (d, $J=16.8 \mathrm{~Hz}, 1 \mathrm{H}), 2.90$ (s, 3H), 2.71 (dd, $J=11.1,4.6 \mathrm{~Hz}, 1 \mathrm{H})$

${ }^{13} \mathrm{C}$ NMR (126 MHz, Acetonitrile- $d_{3}$ )

$\delta 167.8,167.4,161.3,141.0,129.0,128.5,125.9,66.7,62.3,62.3,45.9,29.8$

${ }^{11} \mathrm{~B}$ NMR (128 MHz, Acetonitrile- $d_{3}$ )

$\delta 11.5$

HRMS [DART-MS] $\left(\mathrm{M}+\mathrm{NH}_{4}{ }^{+}\right)$

$m / z$ calculated for $\mathrm{C}_{14} \mathrm{H}_{20} \mathrm{BN}_{2} \mathrm{O}_{6}=323.1414$

$m / z$ found $=323.1421$

TLC (hexanes:acetone 1:2)

$$
\mathrm{R}_{\mathrm{f}}=0.44
$$




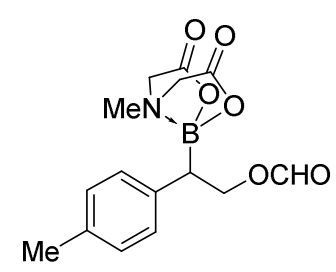

(2-(formyloxy)-1-(p-tolyl)ethyl)MIDA boronate (2b-formate)

$0.114 \mathrm{~g}, 12 \%$ yield, white solid

${ }^{1} \mathrm{H}$ NMR (500 MHz, Acetonitrile- $\left.d_{3}\right)$

$\delta 7.94(\mathrm{~s}, 1 \mathrm{H}), 7.20-7.16(\mathrm{~m}, 2 \mathrm{H}), 7.16-7.11(\mathrm{~m}, 2 \mathrm{H}), 4.62(\mathrm{dd}, J=11.1,4.6 \mathrm{~Hz}, 1 \mathrm{H})$, 4.40 (t, $J=11.1 \mathrm{~Hz}, 1 \mathrm{H}), 3.97(\mathrm{~d}, J=17.2 \mathrm{~Hz}, 1 \mathrm{H}), 3.88(\mathrm{~d}, J=6.7 \mathrm{~Hz}, 1 \mathrm{H}), 3.84$ (d, $J=$ $7.0 \mathrm{~Hz}, 1 \mathrm{H}), 3.20$ (d, $J=16.8 \mathrm{~Hz}, 1 \mathrm{H}), 2.88(\mathrm{~s}, 3 \mathrm{H}), 2.65(\mathrm{dd}, J=11.0,4.5 \mathrm{~Hz}, 1 \mathrm{H}), 2.31$ $(\mathrm{s}, 3 \mathrm{H})$

${ }^{13} \mathrm{C}$ NMR (126 MHz, Acetonitrile- $\left.d_{3}\right)$

$\delta 168.0,167.5,161.4,137.7,135.5,129.1,128.9,66.8,62.3,62.2,45.8,20.0$

${ }^{11} \mathrm{~B}$ NMR (128 MHz, Acetonitrile- $\left.d_{3}\right)$

$\delta 11.5$

HRMS [DART-MS] $\left(\mathrm{M}+\mathrm{NH}_{4}^{+}\right)$

$m / z$ calculated for $\mathrm{C}_{15} \mathrm{H}_{22} \mathrm{BNO}_{6}=337.1570$

$m / z$ found $=337.1579$

TLC (hexanes:acetone 3:4)

$$
\mathrm{R}_{\mathrm{f}}=0.38
$$

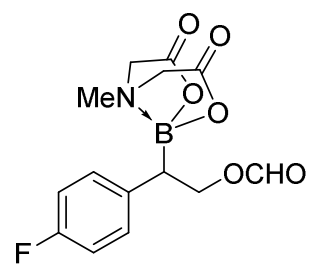

(1-(4-fluorophenyl)-2-(formyloxy)ethyl)MIDA boronate (2c-formate)

$0.164 \mathrm{~g}, 17 \%$ yield, white solid

${ }^{1} \mathrm{H}$ NMR (400 MHz, Acetone- $\left.d_{6}\right)$

$\delta 8.00(\mathrm{~s}, 1 \mathrm{H}), 7.41-7.33(\mathrm{~m}, 2 \mathrm{H}), 7.10-7.02(\mathrm{~m}, 2 \mathrm{H}), 4.64(\mathrm{dd}, J=11.2,4.5 \mathrm{~Hz}, 1 \mathrm{H})$, $4.51-4.41(\mathrm{~m}, 1 \mathrm{H}), 4.29(\mathrm{~d}, J=17.1 \mathrm{~Hz}, 1 \mathrm{H}), 4.22-4.10(\mathrm{~m}, 2 \mathrm{H}), 3.52(\mathrm{~d}, J=16.8 \mathrm{~Hz}$, $1 \mathrm{H}), 3.12(\mathrm{~s}, 3 \mathrm{H}), 2.84-2.80(\mathrm{~m}, 1 \mathrm{H})$

${ }^{13} \mathrm{C}$ NMR (101 MHz, Acetone- $\left.d_{6}\right)$ 
$\delta 168.4,168.1,162.1(\mathrm{~d}, J=482 \mathrm{~Hz}), 160.9,138.2(\mathrm{~d}, J=3.2 \mathrm{~Hz}), 131.6(\mathrm{~d}, J=7.7 \mathrm{~Hz})$, $115.8(\mathrm{~d}, J=20.9 \mathrm{~Hz}), 67.6,63.3,63.3,46.8$

${ }^{11}$ B NMR (128 MHz, Acetone- $d_{6}$ )

$\delta 11.5$

${ }^{19}$ F NMR (282 MHz, Acetone- $d_{6}$ )

$\delta-119.2$

HRMS [DART-MS] $\left(\mathrm{M}+\mathrm{NH}_{4}{ }^{+}\right)$

$m / z$ calculated for $\mathrm{C}_{14} \mathrm{H}_{19} \mathrm{BFN}_{2} \mathrm{O}_{6}=341.1320$

$\mathrm{m} / \mathrm{z}$ found $=341.1324$

TLC (hexanes:acetone 1:2)

$$
\mathrm{R}_{\mathrm{f}}=0.54
$$

\section{General Procedure for the Rh-Catalyzed C-H-Amination of $\alpha$-Boryl Sulfamate Esters}

To a flame dried flask equipped with a reflux condenser was added boryl sulphonamide (1.0 mmol), $\mathrm{MgO}$ ( 2.5 equiv), and $\mathrm{Rh}_{2}(\mathrm{esp})_{2}(2.5 \% \mathrm{~mol})$. The flask was evacuated and refilled with $\mathrm{N}_{2}$ then was added nitrogen sparged $i \operatorname{PrOAc}(0.1 \mathrm{M})$. Upon addition of $i \operatorname{PrOAc}$ the solution acquires a green colour that may or may not persist throughout the reaction. A solution of $\mathrm{PhI}\left(\mathrm{O}_{2} t \mathrm{Bu}\right)_{2}$ in $i \mathrm{PrOAc}(0.1 \mathrm{M}, 1.25$ equiv) was added to the reaction via syringe pump over 1 hour. The syringe was then removed and the flask was warmed to $45^{\circ} \mathrm{C}$ for $12-24$ hours. Upon complete consumption of starting sulfamate ester, as determined by TLC analysis, the reaction was removed from the heat source and allowed to cool to room temperature. The solvent was then removed in vacuo and subsequently purified via $\mathrm{SiO}_{2}$ chromatography resulting in a white solid.

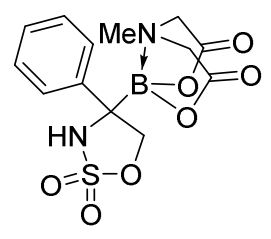

(2,2-dioxido-4-phenyl-1,2,3-oxathiazolidin-4-yl)MIDA boronate (3a)

$0.276 \mathrm{~g}, 78 \%$ yield, white solid

${ }^{1} \mathrm{H}$ NMR (500 MHz, Acetonitrile- $d_{3}$ ) 
$\delta 7.65-7.57(\mathrm{~m}, 2 \mathrm{H}), 7.46-7.38(\mathrm{~m}, 2 \mathrm{H}), 7.38-7.32(\mathrm{~m}, 1 \mathrm{H}), 5.78(\mathrm{~s}, 1 \mathrm{H}), 4.93-4.78$ (m, 2H), $4.05-3.95$ (m, 1H), 3.87 (d, $J=17.5 \mathrm{~Hz}, 1 \mathrm{H}), 3.78$ (d, $J=16.7 \mathrm{~Hz}, 1 \mathrm{H}), 2.86-$ $2.73(\mathrm{~m}, 3 \mathrm{H}), 2.48(\mathrm{~d}, J=16.7 \mathrm{~Hz}, 1 \mathrm{H})$

${ }^{13} \mathrm{C}$ NMR (126 MHz, Acetonitrile- $d_{3}$ )

$\delta 167.5,166.4,138.9,128.6,127.6,126.7,81.5,63.4,62.4,45.5,29.8$

${ }^{11}$ B NMR (128 MHz, Acetonitrile- $d_{3}$ )

$\delta 9.9$

HRMS [DART-MS] $\left(\mathrm{M}+\mathrm{NH}_{4}{ }^{+}\right)$

$m / z$ calculated for $\mathrm{C}_{13} \mathrm{H}_{19} \mathrm{BN}_{3} \mathrm{O}_{7} \mathrm{~S}=372.1036$

$m / z$ found $=372.1031$

TLC (hexanes:acetone 1:1)

$$
\mathrm{R}_{\mathrm{f}}=0.27
$$

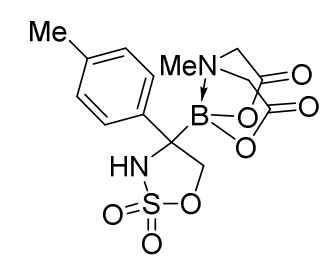

(2,2-dioxido-4-(p-tolyl)-1,2,3-oxathiazolidin-4-yl) MIDA boronate (3b)

$0.268 \mathrm{~g}, 73 \%$ yield, white solid

${ }^{1} \mathrm{H}$ NMR $\left(500 \mathrm{MHz}\right.$, Acetonitrile- $\left.d_{3}\right)$

$\delta 7.59-7.40(\mathrm{~m}, 2 \mathrm{H}), 7.25-7.23(\mathrm{~m}, 2 \mathrm{H}), 5.77(\mathrm{~s}, 1 \mathrm{H}), 4.89-4.83(\mathrm{~m}, 2 \mathrm{H}), 4.02(\mathrm{~d}, J=$ $17.6 \mathrm{~Hz}, 1 \mathrm{H}), 3.88(\mathrm{~d}, J=17.5 \mathrm{~Hz}, 1 \mathrm{H}), 3.80$ (d, $J=16.6 \mathrm{~Hz}, 1 \mathrm{H}), 2.83(\mathrm{~s}, 3 \mathrm{H}), 2.51$ (d, $J$ $=16.7 \mathrm{~Hz}, 1 \mathrm{H}), 2.36(\mathrm{~s}, 3 \mathrm{H})$

${ }^{13} \mathrm{C}$ NMR (126 MHz, Acetonitrile- $d_{3}$ )

$\delta 167.5,166.5,137.6,135.6,129.2,126.6,81.5,63.3,62.4,45.4,29.9,19.9$

${ }^{11} \mathrm{~B}$ NMR (128 MHz, Acetonitrile- $d_{3}$ )

$\delta 9.9$

HRMS [DART-MS] $\left(\mathrm{M}+\mathrm{NH}_{4}{ }^{+}\right)$

$m / z$ calculated for $\mathrm{C}_{14} \mathrm{H}_{21} \mathrm{BN}_{3} \mathrm{O}_{7} \mathrm{~S}=386.1193$

$\mathrm{m} / \mathrm{z}$ found $=386.1206$

TLC (hexanes:acetone 1:2) 


$$
\mathrm{R}_{\mathrm{f}}=0.67
$$

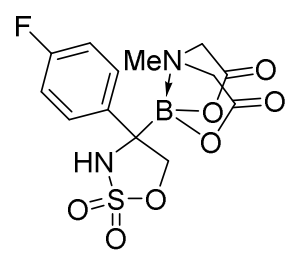

(4-(4-fluorophenyl)-2,2-dioxido-1,2,3-oxathiazolidin-4-yl)MIDA boronate (3c)

$0.301 \mathrm{~g}, 81 \%$, white solid

${ }^{1} \mathrm{H}$ NMR (500 MHz, Acetone- $\left.d_{6}\right)$

$\delta 7.80-7.61(\mathrm{~m}, 2 \mathrm{H}), 7.31-7.07(\mathrm{~m}, 2 \mathrm{H}), 4.98-4.79(\mathrm{~m}, 2 \mathrm{H}), 4.42(\mathrm{~d}, \mathrm{~J}=17.6 \mathrm{~Hz}$, 1H), $4.16-4.08(\mathrm{~m}, 2 \mathrm{H}), 3.17(\mathrm{~s}, 3 \mathrm{H}) 2.88(\mathrm{~d}, \mathrm{~J}=16.8 \mathrm{~Hz}, 1 \mathrm{H})$

${ }^{13} \mathrm{C}$ NMR (126 MHz, Acetone- $\left.d_{6}\right)$

$\delta 172.7,171.4,167.1(\mathrm{~d}, J=244.7 \mathrm{~Hz}), 140.8(\mathrm{~d}, J=3.1 \mathrm{~Hz}), 134.1(\mathrm{~d}, J=8.2 \mathrm{~Hz}), 120.3$ (d, $J=21.5 \mathrm{~Hz}), 86.4,68.9,68.0,50.8$

${ }^{11} \mathrm{~B}$ NMR (128 MHz, Acetone- $\left.d_{6}\right)$

$\delta 10.1$

${ }^{19}$ F NMR (377 MHz, Acetone- $\left.d_{6}\right)$

$\delta-117.13$

HRMS [DART-MS] $\left(\mathrm{M}+\mathrm{NH}_{4}^{+}\right)$

$m / z$ calculated for $\mathrm{C}_{13} \mathrm{H}_{18} \mathrm{BFN}_{3} \mathrm{O}_{7} \mathrm{~S}=390.0942$

$\mathrm{m} / \mathrm{z}$ found $=390.0943$

TLC (hexanes:acetone 1:1)

$$
\mathrm{R}_{\mathrm{f}}=0.37
$$

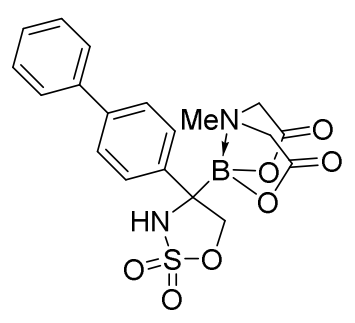

(4-([1,1'-biphenyl]-4-yl)-2,2-dioxido-1,2,3-oxathiazolidin-4-yl)MIDA boronate (3d)

$0.305 \mathrm{~g}, 71 \%$ yield, white solid

${ }^{1} \mathrm{H}$ NMR (400 MHz, Acetonitrile- $d_{3}$ ) 
$\delta 7.80-7.64(\mathrm{~m}, 6 \mathrm{H}), 7.57-7.46(\mathrm{~m}, 2 \mathrm{H}), 7.46-7.36(\mathrm{~m}, 1 \mathrm{H}), 5.87(\mathrm{~s}, 1 \mathrm{H}), 5.01-4.84$ (m, 2H), $4.06(\mathrm{~d}, J=17.6 \mathrm{~Hz}, 1 \mathrm{H}), 3.93(\mathrm{~d}, J=17.6 \mathrm{~Hz}, 1 \mathrm{H}), 3.84(\mathrm{~d}, J=16.7 \mathrm{~Hz}, 1 \mathrm{H})$, $2.87(\mathrm{~s}, 3 \mathrm{H}), 2.69(\mathrm{~d}, J=16.7 \mathrm{~Hz}, 1 \mathrm{H})$

${ }^{13} \mathrm{C}$ NMR (126 MHz, Acetonitrile- $d_{3}$ )

$\delta 167.5,166.5,140.1,139.8,138.0,128.9,127.6,127.3,127.0,126.8,104.9,81.4,63.5$, $62.5,45.6$

${ }^{11}$ B NMR (128 MHz, Acetonitrile- $d_{3}$ )

$\delta 10.0$

HRMS [ESI-MS] $\left(\mathrm{M}^{+}\right)$

$m / z$ calculated for $\mathrm{C}_{19} \mathrm{H}_{19} \mathrm{BN}_{2} \mathrm{O}_{7} \mathrm{~S}=430.1116$

$\mathrm{m} / \mathrm{z}$ found $=430.1115$

TLC (hexanes:acetone 1:2)

$$
\mathrm{R}_{\mathrm{f}}=0.51
$$

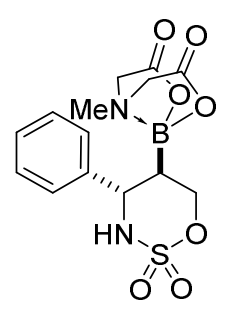

anti-((4S,5R)-2,2-dioxido-4-phenyl-1,2,3-oxathiazinan-5-yl)MIDA boronate (3e)

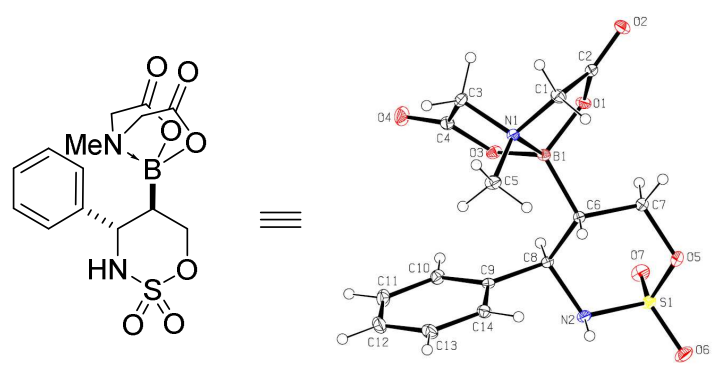

X-ray quality crystals were grown by slow evaporation from a solution of $\mathbf{3 e}$ in hexanes:acetone

$0.213 \mathrm{~g}, 58 \%$ yield, white solid

$\mathrm{dr}=>20: 1$ (anti:syn)

${ }^{1} \mathrm{H}$ NMR (399 MHz, Acetone- $d_{6}$ )

$\delta 7.64-7.52(\mathrm{~m}, 2 \mathrm{H}), 7.47-7.32(\mathrm{~m}, 3 \mathrm{H}), 6.01(\mathrm{~d}, J=8.7 \mathrm{~Hz}, 1 \mathrm{H}), 4.81-4.64(\mathrm{~m}, 3 \mathrm{H})$, $4.11(\mathrm{~d}, J=17.3 \mathrm{~Hz}, 1 \mathrm{H}), 3.98(\mathrm{~d}, J=17.3 \mathrm{~Hz}, 1 \mathrm{H}), 3.82(\mathrm{~d}, J=16.5 \mathrm{~Hz}, 1 \mathrm{H}), 2.84$ (s, $3 \mathrm{H}), 2.30(\mathrm{td}, J=11.8,5.7 \mathrm{~Hz}, 1 \mathrm{H}), 2.22(\mathrm{~d}, J=16.5 \mathrm{~Hz}, 1 \mathrm{H})$

${ }^{13} \mathrm{C}$ NMR (100 MHz, Acetone- $d_{6}$ ) 
$\delta 167.3,166.5,139.7,129.0,128.7,128.7,75.4,63.0,62.6,61.7,46.1$

${ }^{11}$ B NMR $\left(128 \mathrm{MHz}\right.$, Acetone- $\left.d_{6}\right)$

$\delta 11.2$

IR (thin film, $\mathrm{NaCl}$ )

v $3265,2956,1766,1456,1431,1367,1342,1300,1247,1186,1105,1058,1024,1003$, 704

HRMS [DART-MS] $\left(\mathrm{M}+\mathrm{NH}_{4}{ }^{+}\right)$

$m / z$ calculated for $\mathrm{C}_{14} \mathrm{H}_{21} \mathrm{BN}_{3} \mathrm{O}_{7} \mathrm{~S}=386.1193$

$\mathrm{m} / \mathrm{z}$ found $=386.1192$

TLC (hexanes:acetone 1:2)

$$
\mathrm{R}_{\mathrm{f}}=0.29
$$

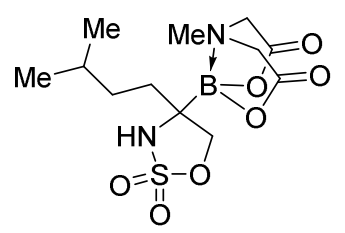

(4-isopentyl-2,2-dioxido-1,2,3-oxathiazolidin-4-yl)MIDA boronate (3f')

$0.070 \mathrm{~g}, 20 \%$ yield, white solid

Product ratio $1.7: 16$ vs 5

${ }^{1} \mathrm{H}$ NMR (400 MHz, Acetonitrile- $d_{3}$ )

$\delta 4.58(\mathrm{~d}, J=9.5 \mathrm{~Hz}, 1 \mathrm{H}), 4.47(\mathrm{~d}, J=9.6 \mathrm{~Hz}, 1 \mathrm{H}), 4.03(\mathrm{~d}, J=5.8 \mathrm{~Hz}, 1 \mathrm{H}), 3.99(\mathrm{~d}, J=$ $6.0 \mathrm{~Hz}, 1 \mathrm{H}), 3.94$ (d, $J=2.3 \mathrm{~Hz}, 2 \mathrm{H}), 3.90(\mathrm{~d}, J=2.7 \mathrm{~Hz}, 1 \mathrm{H}), 3.13$ (s, 3H), $1.83-1.64$ $(\mathrm{m}, 2 \mathrm{H}), 1.56-1.47(\mathrm{~m}, 1 \mathrm{H}), 1.40-1.22(\mathrm{~m}, 2 \mathrm{H}), 0.92(\mathrm{~d}, J=2.8 \mathrm{~Hz}, 3 \mathrm{H}), 0.91(\mathrm{~d}, J=$ $2.8 \mathrm{~Hz}, 3 \mathrm{H})$

${ }^{13} \mathrm{C}$ NMR (101 MHz, Acetonitrile- $d_{3}$ )

$\delta 167.0,166.5,73.3,62.5,62.1,45.1,33.4,31.5,27.8,21.4,21.1$

${ }^{11}$ B NMR (128 MHz, Acetonitrile- $d_{3}$ )

$\delta 9.9$

HRMS [DART-MS] $\left(\mathrm{M}+\mathrm{NH}_{4}{ }^{+}\right)$

$m / z$ calculated for $\mathrm{C}_{12} \mathrm{H}_{25} \mathrm{BN}_{3} \mathrm{O}_{7} \mathrm{~S}=366.1506$

$\mathrm{m} / \mathrm{z}$ found $=366.1506$

TLC (hexanes:acetone 1:2) 


$$
\mathrm{R}_{\mathrm{f}}=0.39
$$

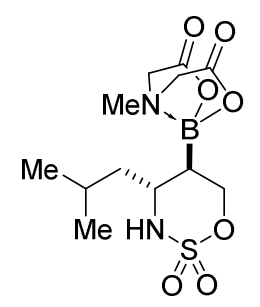

anti-4-isobutyl-2,2-dioxido-1,2,3-oxathiazinan-5-yl) MIDA boronate (anti-3f)

$0.111 \mathrm{~g}, 32 \%$ combined yield, white solid

$\mathrm{dr}=2: 1($ anti: syn)

anti-diastereomer

${ }^{1} \mathrm{H}$ NMR (500 MHz, Acetonitrile- $\left.d_{3}\right)$

$\delta 5.33(\mathrm{~d}, J=6.2 \mathrm{~Hz}, 1 \mathrm{H}), 4.81-4.70(\mathrm{~m}, 1 \mathrm{H}), 4.51(\mathrm{dd}, J=12.3,3.6 \mathrm{~Hz}, 1 \mathrm{H}), 4.02(\mathrm{~d}, J$ $=16.0 \mathrm{~Hz}, 1 \mathrm{H}), 3.98(\mathrm{~d}, J=16.0 \mathrm{~Hz}, 1 \mathrm{H}), 3.90(\mathrm{~d}, J=17.0 \mathrm{~Hz}, 1 \mathrm{H}), 3.83(\mathrm{~d}, J=17.0 \mathrm{~Hz}$, $1 \mathrm{H}), 3.69-3.59(\mathrm{~m}, 1 \mathrm{H}), 2.99(\mathrm{~s}, 3 \mathrm{H}), 1.91-1.78(\mathrm{~m}, 2 \mathrm{H}), 1.20-1.11(\mathrm{~m}, 1 \mathrm{H}), 0.95(\mathrm{~d}$, $J=6.8 \mathrm{~Hz}, 3 \mathrm{H}), 0.88(\mathrm{~d}, J=6.6 \mathrm{~Hz}, 3 \mathrm{H})$

${ }^{13} \mathrm{C}$ NMR (101 MHz, Acetonitrile- $\left.d_{3}\right)$

$\delta 168.4,168.3,73.5,62.9,62.5,56.8,46.8,38.0,25.3,24.0$

${ }^{11} \mathrm{~B}$ NMR (128 MHz, Acetonitrile- $\left.d_{3}\right)$

$\delta 11.0$

HRMS [DART-MS] $\left(\mathrm{M}+\mathrm{NH}_{4}{ }^{+}\right)$

$m / z$ calculated for $\mathrm{C}_{12} \mathrm{H}_{21} \mathrm{BN}_{2} \mathrm{O}_{7} \mathrm{~S}=366.1506$

$\mathrm{m} / \mathrm{z}$ found $=366.1523$

TLC (hexanes:acetone 1:2)

$$
\mathrm{R}_{\mathrm{f}}=0.26
$$

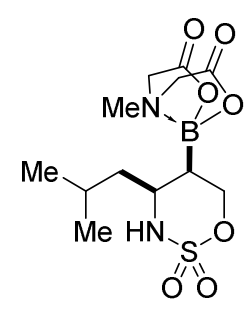

syn-4-isobutyl-2,2-dioxido-1,2,3-oxathiazinan-5-yl) MIDA boronate (syn-3f)

syn-diastereomer

${ }^{1} \mathrm{H}$ NMR (500 MHz, Acetonitrile- $\left.d_{3}\right)-2$ conformations, major peaks reported 
$\delta 5.03-4.95(\mathrm{~m}, 1 \mathrm{H}), 4.61-4.51(\mathrm{~m}, 2 \mathrm{H}), 4.07-3.95(\mathrm{~m}, 2 \mathrm{H}), 3.95-3.82(\mathrm{~m}, 2 \mathrm{H})$, $3.71-3.65(\mathrm{~m}, 1 \mathrm{H}), 3.00(\mathrm{~s}, 3 \mathrm{H}), 1.92-1.92(\mathrm{~m}, 1 \mathrm{H}), 1.62-1.50(\mathrm{~m}, 1 \mathrm{H}), 1.42-1.34$ $(\mathrm{m}, 1 \mathrm{H}), 0.99-0.94(\mathrm{~m}, 1 \mathrm{H}), 0.94-0.87(\mathrm{~m}, 6 \mathrm{H})$

${ }^{13} \mathrm{C}$ NMR (126 MHz, Acetonitrile- $\left.d_{3}\right)-2$ conformations

$\delta 167.9,167.7,167.6,167.1,74.9,74.5,62.5,62.5,56.5,55.8,46.6,45.8,43.1$ (2C), $28.4,27.7,23.7,22.9,22.6,19.9$

${ }^{11} \mathrm{~B}$ NMR (128 MHz, Acetonitrile- $\left.d_{3}\right)$

$\delta 11.2$

HRMS [DART-MS] $\left(\mathrm{M}+\mathrm{NH}_{4}{ }^{+}\right)$

$m / z$ calculated for $\mathrm{C}_{12} \mathrm{H}_{25} \mathrm{BN}_{3} \mathrm{O}_{7} \mathrm{~S}=366.1506$

$\mathrm{m} / \mathrm{z}$ found $=366.1523$

TLC (hexanes:acetone 1:2)

$$
\mathrm{R}_{\mathrm{f}}=0.18
$$

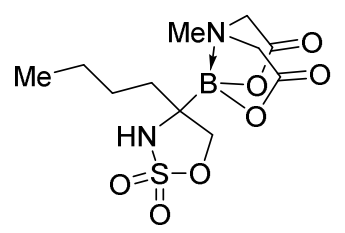

(4-butyl-2,2-dioxido-1,2,3-oxathiazolidin-4-yl)MIDA boronate (3g')

$0.056 \mathrm{~g}, 17 \%$ yield, white solid

${ }^{1} \mathrm{H}$ NMR (400 MHz, Acetonitrile- $d_{3}$ )

$\delta 5.42(\mathrm{~s}, 1 \mathrm{H}), 4.62(\mathrm{~d}, J=9.5 \mathrm{~Hz}, 1 \mathrm{H}), 4.52(\mathrm{~d}, J=9.5 \mathrm{~Hz}, 1 \mathrm{H}), 4.08-4.01(\mathrm{~m}, 2 \mathrm{H})$, $3.98-3.93(\mathrm{~m}, 2 \mathrm{H}), 3.16(\mathrm{~s}, 3 \mathrm{H}), 1.84-1.66(\mathrm{~m}, 2 \mathrm{H}), 1.53-1.26(\mathrm{~m}, 4 \mathrm{H}), 0.97(\mathrm{t}, J=$ $7.1 \mathrm{~Hz}, 3 \mathrm{H})$

${ }^{13} \mathrm{C}$ NMR (101 MHz, Acetonitrile- $\left.d_{3}\right)$

$\delta 167.0,166.5,73.4,62.5,62.0,45.1,35.2,24.91,22.3,12.7$

${ }^{11} \mathrm{~B}$ NMR (128 MHz, Acetonitrile- $\left.d_{3}\right)$

$\delta 10.0$

IR (KBr, thin film)

v $3277,3265,2958,2874,1760,1751,1627,1460,1340,1296,1249,1182,1151,1097$, $1037,995,960,895,873$

HRMS [DART-MS] $\left(\mathrm{M}+\mathrm{H}^{+}\right)$

$m / z$ calculated for $\mathrm{C}_{11} \mathrm{H}_{20} \mathrm{BN}_{2} \mathrm{O}_{7} \mathrm{~S}=335.1078$

$m / z$ found $=335.1075$ 
TLC (EtOAc:MeCN 9:1)

$$
\mathrm{R}_{\mathrm{f}}=0.7
$$

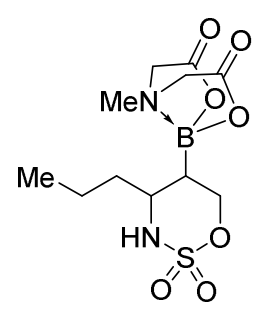

(2,2-dioxido-4-propyl-1,2,3-oxathiazinan-5-yl)MIDA boronate (3g)

$0.101 \mathrm{~g}, 29 \%$ combined yield, white solid

d.r. $=1: 1$

Diastereomer A:

${ }^{1} \mathrm{H}$ NMR (400 MHz, Acetonitrile- $\left.d_{3}\right)$

$\delta 5.35-5.28(\mathrm{~m}, 1 \mathrm{H}), 4.80(\mathrm{dd}, J=12.2,11.2 \mathrm{~Hz}, 1 \mathrm{H}), 4.63-4.50(\mathrm{~m}, 1 \mathrm{H}), 4.08-3.79$ (m, 4H) $3.66-3.54(\mathrm{~m}, 1 \mathrm{H}), 3.03(\mathrm{~s}, 3 \mathrm{H}), 2.30-2.15(\mathrm{~m}, 2 \mathrm{H}), 1.87-1.82(\mathrm{~m}, 1 \mathrm{H}), 1.75$ $-1.58(\mathrm{~m}, 1 \mathrm{H}), 1.53-1.31(\mathrm{~m}, 1 \mathrm{H}), 0.97(\mathrm{t}, J=7.3 \mathrm{~Hz}, 3 \mathrm{H})$

${ }^{13} \mathrm{C}$ NMR $\left(101 \mathrm{MHz}\right.$, Acetonitrile- $\left.d_{3}\right)$

$\delta 167.4,167.3,72.4,61.8,61.5,57.7,45.7,30.5,28.6,19.4,12.8$

${ }^{11} \mathrm{~B}$ NMR (128 MHz, Acetonitrile- $d_{3}$ )

$\delta 11.0$

HRMS [ESI-MS] $\left(\mathrm{M}+\mathrm{H}^{+}\right)$

$m / z$ calculated for $\mathrm{C}_{11} \mathrm{H}_{20} \mathrm{BN}_{2} \mathrm{O}_{7} \mathrm{~S}=335.1078$

$m / z$ found $=335.1075$

TLC (EtOAc:MeCN 9:1)

$$
\mathrm{R}_{\mathrm{f}}=0.61
$$

Diastereoisomer B

${ }^{1} \mathrm{H}$ NMR (400 MHz, Acetonitrile- $d_{3}$ )

$\delta 4.92(\mathrm{~d}, J=9.4 \mathrm{~Hz}, 1 \mathrm{H}), 4.62-4.46(\mathrm{~m}, 2 \mathrm{H}), 4.04-3.91(\mathrm{~m}, 2 \mathrm{H}), 3.93-3.77(\mathrm{~m}, 2 \mathrm{H})$, $3.60(\mathrm{~m}, 1 \mathrm{H}), 2.97(\mathrm{~s}, 3 \mathrm{H}), 2.18(\mathrm{~s}, 2 \mathrm{H}), 1.70-1.47(\mathrm{~m}, 2 \mathrm{H}), 1.50-1.28(\mathrm{~m}, 1 \mathrm{H}), 0.89(\mathrm{t}$, $J=7.3 \mathrm{~Hz}, 3 \mathrm{H})$

${ }^{13} \mathrm{C}$ NMR (101 MHz, Acetonitrile- $d_{3}$ )

$\delta 167.4,166.8,74.0,62.1,62.1,57.7,46.1,35.7,28.4,18.0,12.4$ 
${ }^{11}$ B NMR (128 MHz, Acetonitrile- $\left.d_{3}\right)$

$\delta 11.2$

HRMS [ESI-MS] $\left(\mathrm{M}+\mathrm{H}^{+}\right)$

$m / z$ calculated for $\mathrm{C}_{11} \mathrm{H}_{20} \mathrm{BN}_{2} \mathrm{O}_{7} \mathrm{~S}=335.1078$

$\mathrm{m} / \mathrm{z}$ found $=335.1075$

TLC (EtOAc:MeCN 9:1)

$$
\mathrm{R}_{\mathrm{f}}=0.54
$$

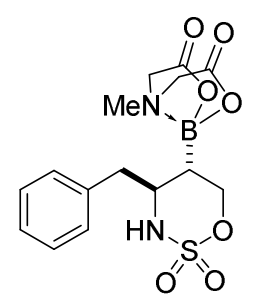

syn-((4S,5S)-4-benzyl-2,2-dioxido-1,2,3-oxathiazinan-5-yl)MIDA boronate (syn-3h)

$0.145 \mathrm{~g}, 38 \%$ yield, white solid

d.r. $=4: 1$ (anti:syn)

${ }^{1} \mathrm{H}$ NMR $\left(500 \mathrm{MHz}\right.$, Acetonitrile- $\left.d_{3}\right)+$ minor contamination with other diastereomer

$\delta 7.42-7.33(\mathrm{~m}, 2 \mathrm{H}), 7.31-7.24(\mathrm{~m}, 3 \mathrm{H}), 5.14(\mathrm{~d}, J=9.2 \mathrm{~Hz}, 1 \mathrm{H}), 4.73-4.65(\mathrm{~m}, 1 \mathrm{H})$, 4. $62-4.57$ (m, 1H), 4.05 (dd, $J=17.3,1.5 \mathrm{~Hz}, 2 \mathrm{H}), 3.98-3.90$ (m, 1H), 3.87 (dd, $J=$ $17.1,0.4 \mathrm{~Hz}, 1 \mathrm{H}$ ), 3.16 (dd, $J=13.9,3.9 \mathrm{~Hz}, 1 \mathrm{H}), 2.95$ (s, 3H), 2.78 (dd, $J=13.9,10.5$

$\mathrm{Hz}, 1 \mathrm{H}), 1.57$ (ddd, $J=10.5,8.8,4.5 \mathrm{~Hz}, 1 \mathrm{H}$ )

${ }^{13} \mathrm{C}$ NMR (126 MHz, Acetonitrile- $d_{3}$ )

$\delta 167.9,167.0,138.5,129.3,128.3,126.4,74.1,62.4,60.0,46.4,40.1$

${ }^{11}$ B NMR (128 MHz, Acetonitrile- $d_{3}$ )

$\delta 11.1$

HRMS [DART-MS] $\left(\mathrm{M}+\mathrm{NH}_{4}{ }^{+}\right)$

$m / z$ calculated for $\mathrm{C}_{14} \mathrm{H}_{21} \mathrm{BN}_{3} \mathrm{O}_{7} \mathrm{~S}=386.1193$

$\mathrm{m} / \mathrm{z}$ found $=386.1192$

TLC (hexanes:acetone 1:3)

$$
\mathrm{R}_{\mathrm{f}}=0.44
$$




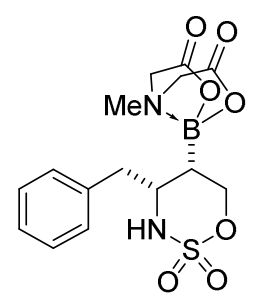

((4R,5S)-4-benzyl-2,2-dioxido-1,2,3-oxathiazinan-5-yl)MIDA boronate (anti-3h)

$0.060 \mathrm{~g}, 16 \%$ yield, white solid

${ }^{1} \mathrm{H}$ NMR $\left(500 \mathrm{MHz}\right.$, Acetonitrile- $\left.d_{3}\right)+$ minor contamination with other diastereomer

$\delta 7.34-7.30(\mathrm{~m}, 3 \mathrm{H}), 7.27-7.17(\mathrm{~m}, 2 \mathrm{H}), 5.23(\mathrm{~d}, J=6.1 \mathrm{~Hz}, 1 \mathrm{H}), 4.91-4.80(\mathrm{~m}, 1 \mathrm{H})$, $4.62-4.54(\mathrm{~m}, 1 \mathrm{H}), 4.09-4.02(\mathrm{~m}, 2 \mathrm{H}), 3.92(\mathrm{~d}, J=17.0 \mathrm{~Hz}, 1 \mathrm{H}), 3.86(\mathrm{~d}, J=17.3 \mathrm{~Hz}$, $1 \mathrm{H}), 3.79-3.71(\mathrm{~m}, 1 \mathrm{H}), 3.52-3.47(\mathrm{~m}, 1 \mathrm{H}), 3.03(\mathrm{~s}, 3 \mathrm{H}), 2.87-2.81(\mathrm{~m}, 1 \mathrm{H}), 1.93-$ $1.89(\mathrm{~m}, 1 \mathrm{H})$

${ }^{13} \mathrm{C}$ NMR $\left(126 \mathrm{MHz}\right.$, Acetonitrile- $\left.d_{3}\right)$

$\delta 167.6,167.3,139.9,129.4,128.1,126.1,72.5,62.0,61.7,60.2,45.9,34.3$

${ }^{11}$ B NMR (128 MHz, Acetonitrile- $d_{3}$ )

$\delta 11.0$

HRMS [ESI-MS] $\left(\mathrm{M}+\mathrm{NH}_{4}{ }^{+}\right)$

$m / z$ calculated for $\mathrm{C}_{15} \mathrm{H}_{23} \mathrm{BN}_{3} \mathrm{O}_{7} \mathrm{~S}=400.1381$

$m / z$ found $=400.1379$

TLC (hexanes:acetone 1:3)

$$
\mathrm{R}_{\mathrm{f}}=0.41
$$

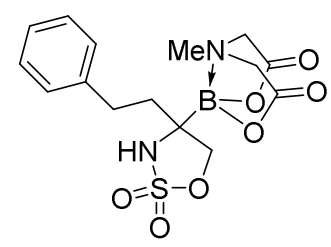

(2,2-dioxido-4-phenethyl-1,2,3-oxathiazolidin-4-yl)MIDA boronate (3h')

$0.145 \mathrm{~g}, 38 \%$ yield, white solid

${ }^{1} \mathrm{H}$ NMR $\left(500 \mathrm{MHz}\right.$, Acetonitrile- $\left.d_{3}\right)$

$\delta 7.35-7.26(\mathrm{~m}, 4 \mathrm{H}), 7.25-7.15(\mathrm{~m}, 1 \mathrm{H}), 5.68(\mathrm{~s}, 1 \mathrm{H}), 4.73-4.62(\mathrm{~m}, 2 \mathrm{H}), 4.09-4.02$ (m, 2H), $3.98(\mathrm{~d}, J=10.1 \mathrm{~Hz}, 1 \mathrm{H}), 3.95(\mathrm{~d}, J=9.9 \mathrm{~Hz}, 1 \mathrm{H}), 3.17$ (s, 3H), $2.86-2.71(\mathrm{~m}$, $2 \mathrm{H}), 2.08-1.98(\mathrm{~m}, 2 \mathrm{H})$

${ }^{13} \mathrm{C}$ NMR (126 MHz, Acetonitrile- $d_{3}$ ) 
$\delta 167.5,167.1,141.8,128.4,128.3,125.9,73.8,63.1,62.6,45.7,38.2,29.5$

${ }^{11}$ B NMR (128 MHz, Acetonitrile- $d_{3}$ )

$\delta 9.9$

HRMS [DART-MS] $\left(\mathrm{M}+\mathrm{NH}_{4}{ }^{+}\right)$

$m / z$ calculated for $\mathrm{C}_{15} \mathrm{H}_{23} \mathrm{BN}_{3} \mathrm{O}_{7} \mathrm{~S}=400.1375$

$\mathrm{m} / \mathrm{z}$ found $=400.1375$

TLC (hexanes:acetone 1:3)

$$
\mathrm{R}_{\mathrm{f}}=0.63
$$

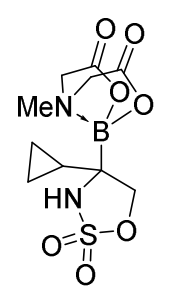

(4-cyclopropyl-2,2-dioxido-1,2,3-oxathiazolidin-4-yl)MIDA boronate (3i)

$0.187 \mathrm{~g}, 59 \%$ yield, white solid

${ }^{1} \mathrm{H}$ NMR (500 MHz, Acetonitrile- $d_{3}$ )

$\delta 5.33(\mathrm{~s}, 1 \mathrm{H}), 4.54(\mathrm{~d}, J=9.2 \mathrm{~Hz}, 1 \mathrm{H}), 4.24(\mathrm{~d}, J=9.2 \mathrm{~Hz}, 1 \mathrm{H}), 4.15-4.11(\mathrm{~m}, 1 \mathrm{H})$, $4.11-4.06$ (m, 1H), $4.02-3.94(\mathrm{~m}, 2 \mathrm{H}), 3.15-3.10(\mathrm{~m}, 3 \mathrm{H}), 0.8-0.74(\mathrm{~m}, 1 \mathrm{H}), 0.65-$ $0.47(\mathrm{~m}, 4 \mathrm{H})$

${ }^{13} \mathrm{C}$ NMR (126 MHz, Acetonitrile- $d_{3}$ )

$\delta 167.5,166.9,75.2,63.3,62.7,46.0,14.0,1.8$

${ }^{11} \mathrm{~B}$ NMR (128 MHz, Acetonitrile- $d_{3}$ )

$\delta 10.4$

HRMS [ESI-MS] $\left(\mathrm{M}+\mathrm{H}^{+}\right)$

$m / z$ calculated for $\mathrm{C}_{10} \mathrm{H}_{16} \mathrm{BN}_{2} \mathrm{O}_{7} \mathrm{~S}=319.0771$

$\mathrm{m} / \mathrm{z}$ found $=319.0766$

TLC (hexanes:acetone 1:2)

$$
\mathrm{R}_{\mathrm{f}}=0.35
$$




\section{${ }^{1} \mathrm{H}$ and ${ }^{13} \mathrm{C}$ NMR Spectra}




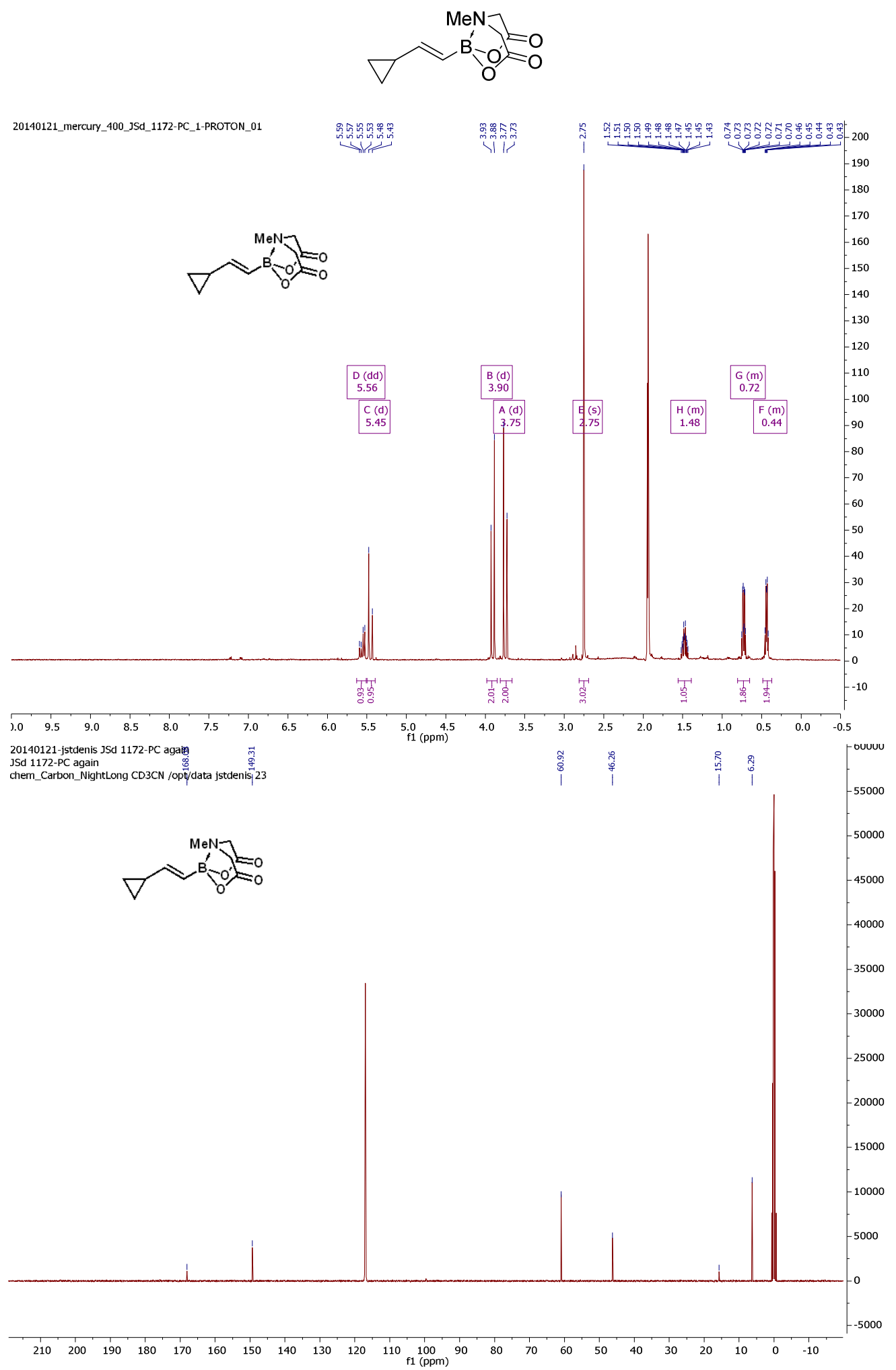




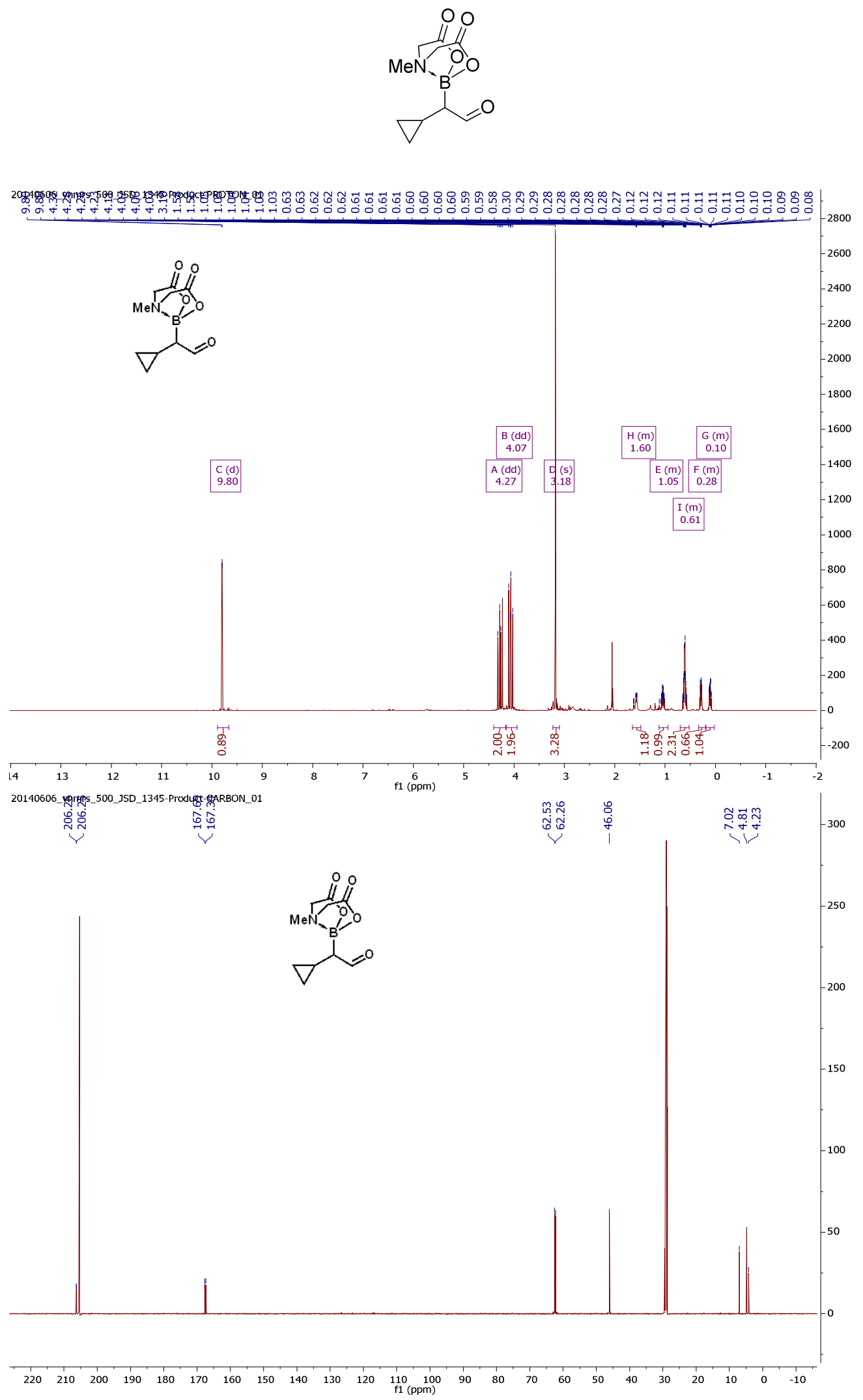




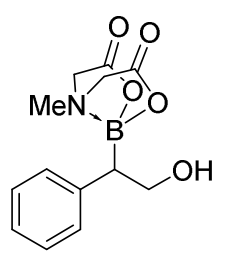

$1 a$

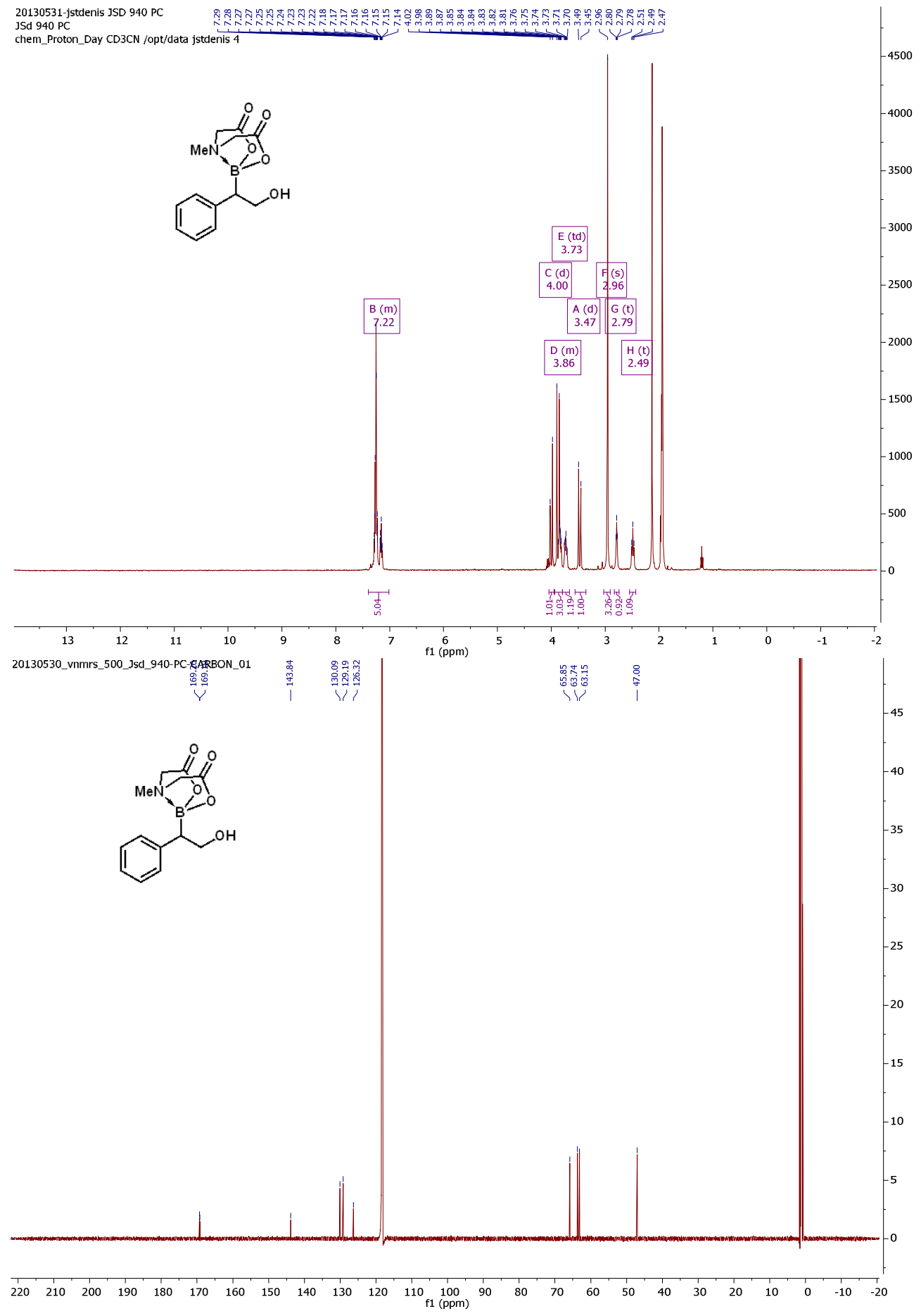




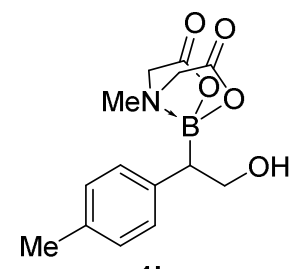

1b

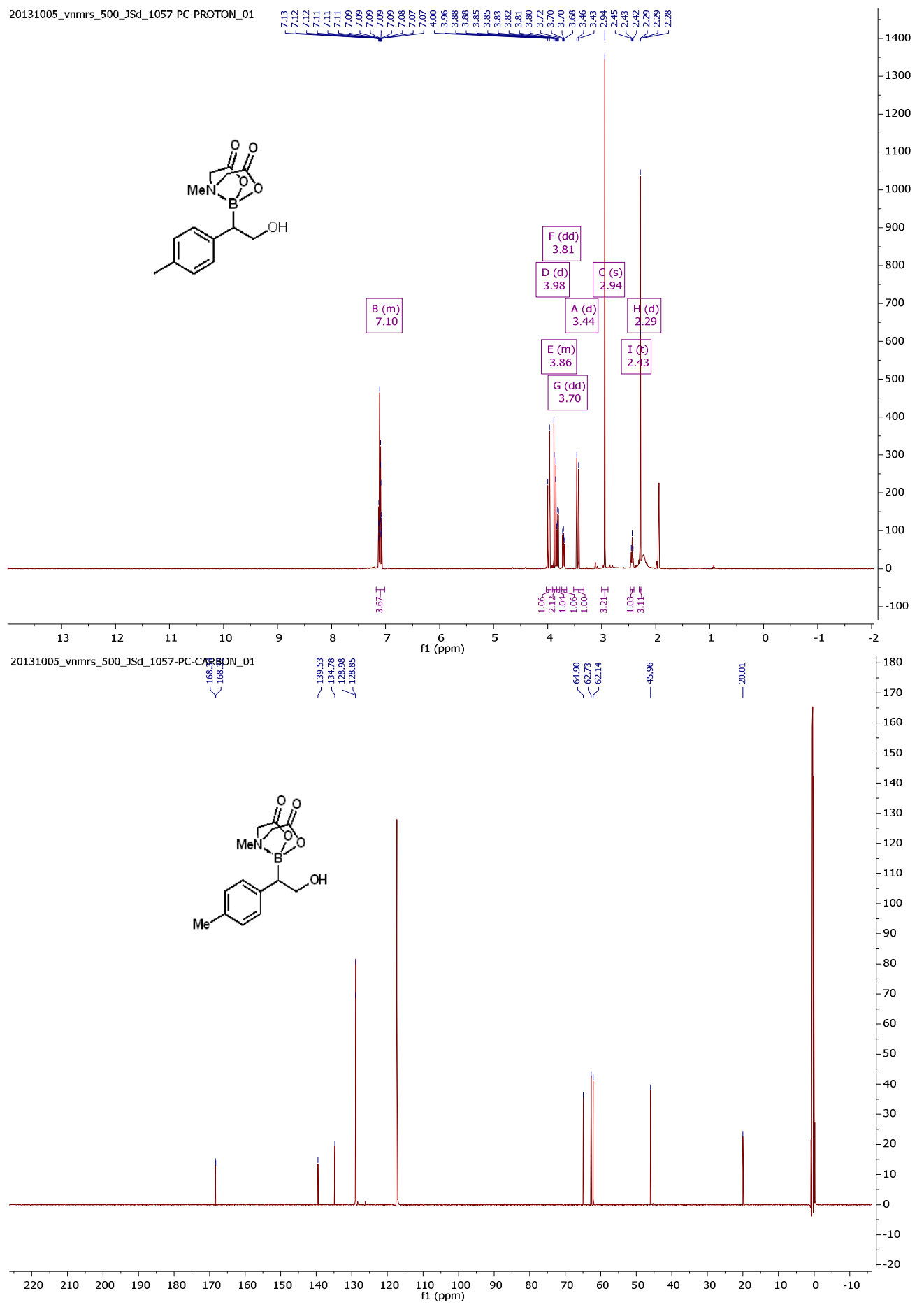



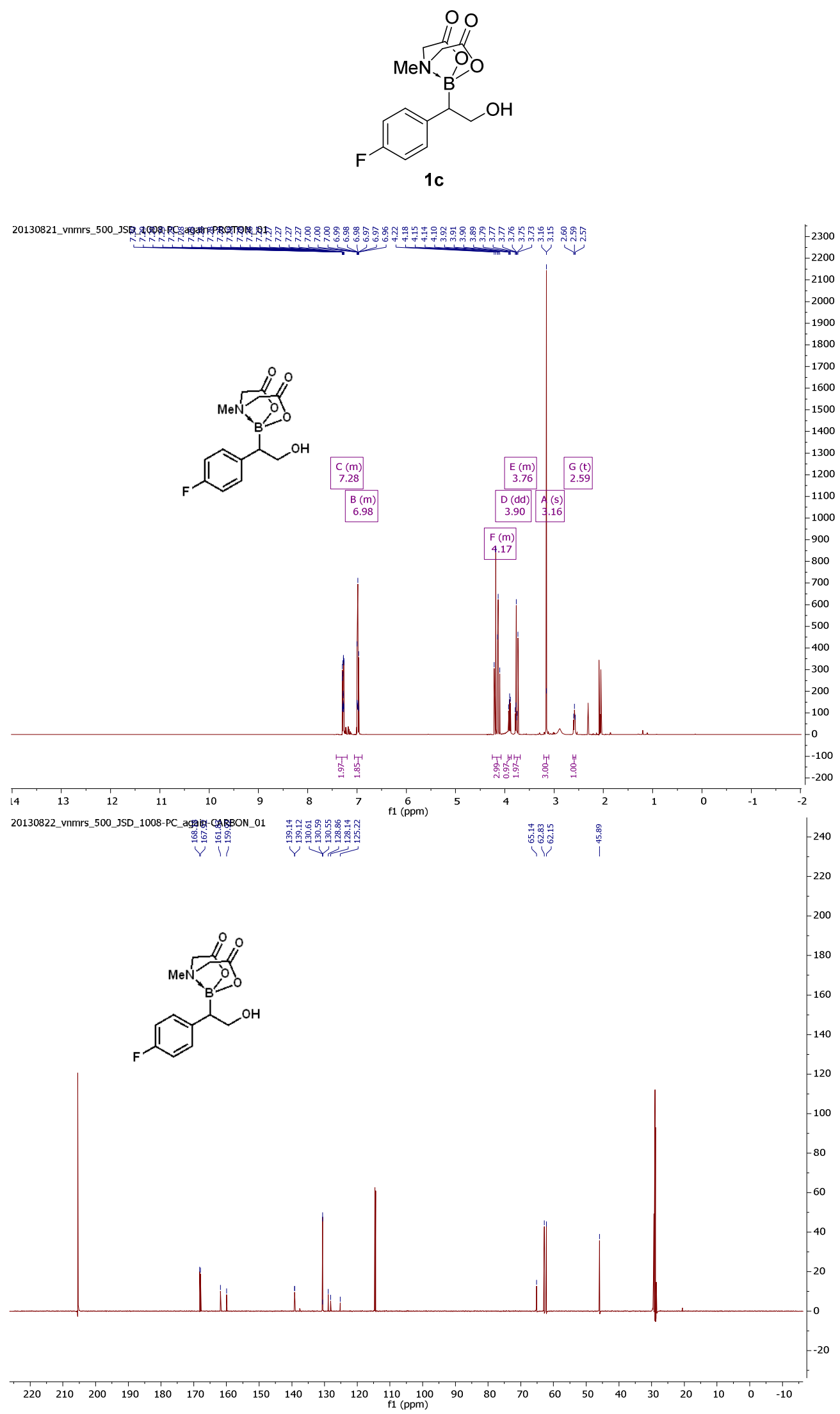

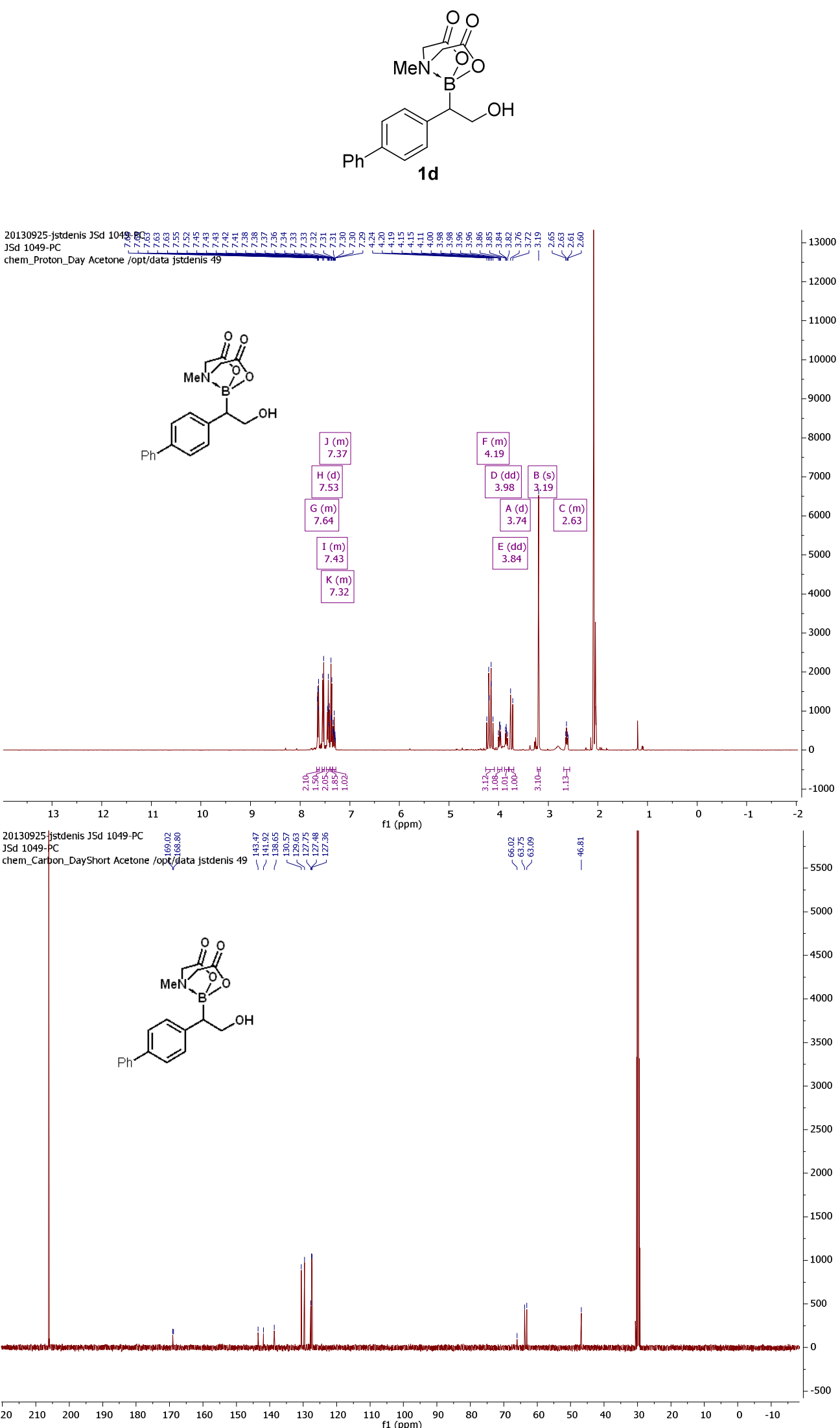


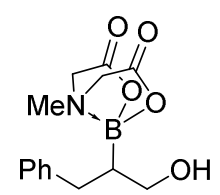

$1 e$

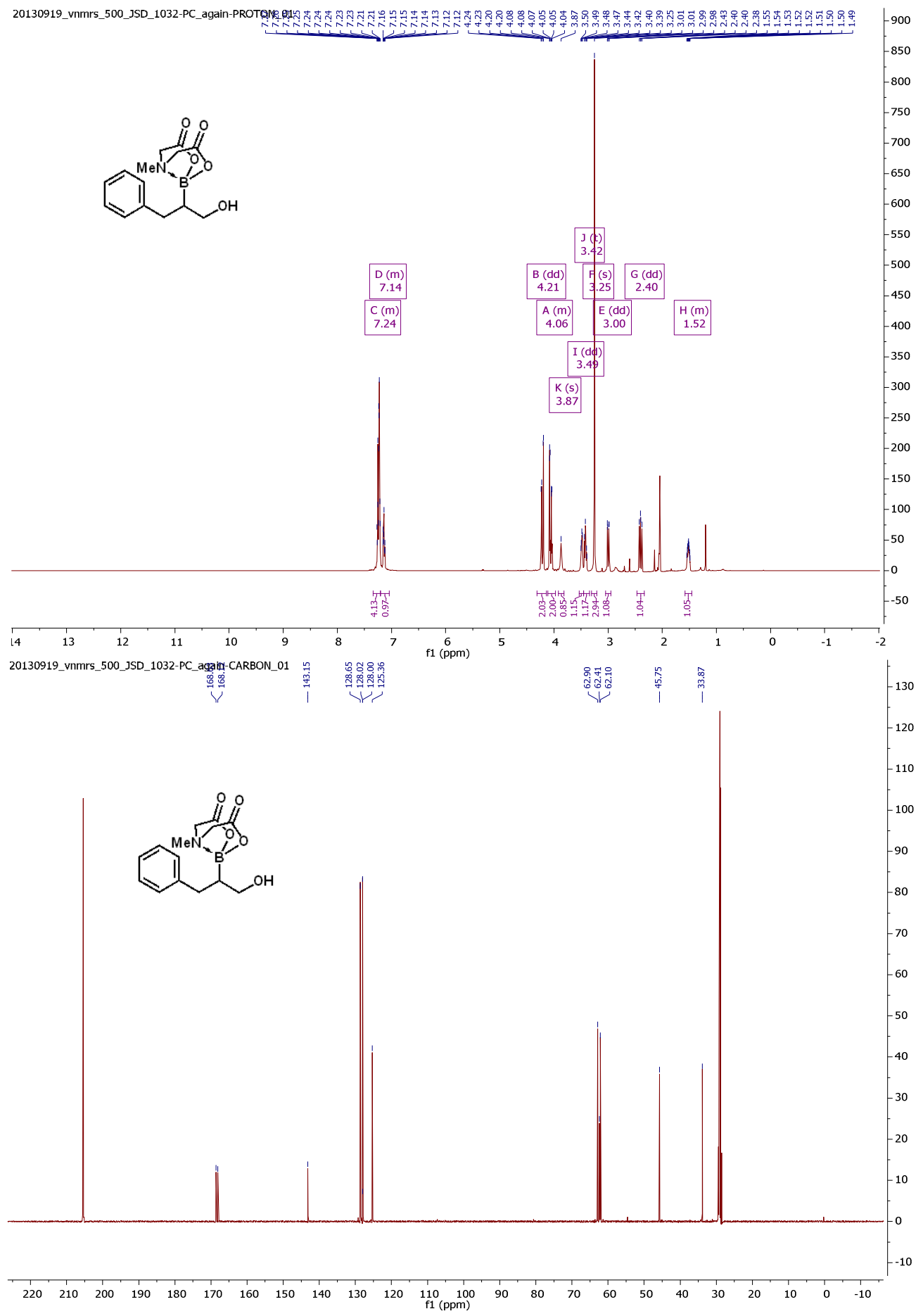




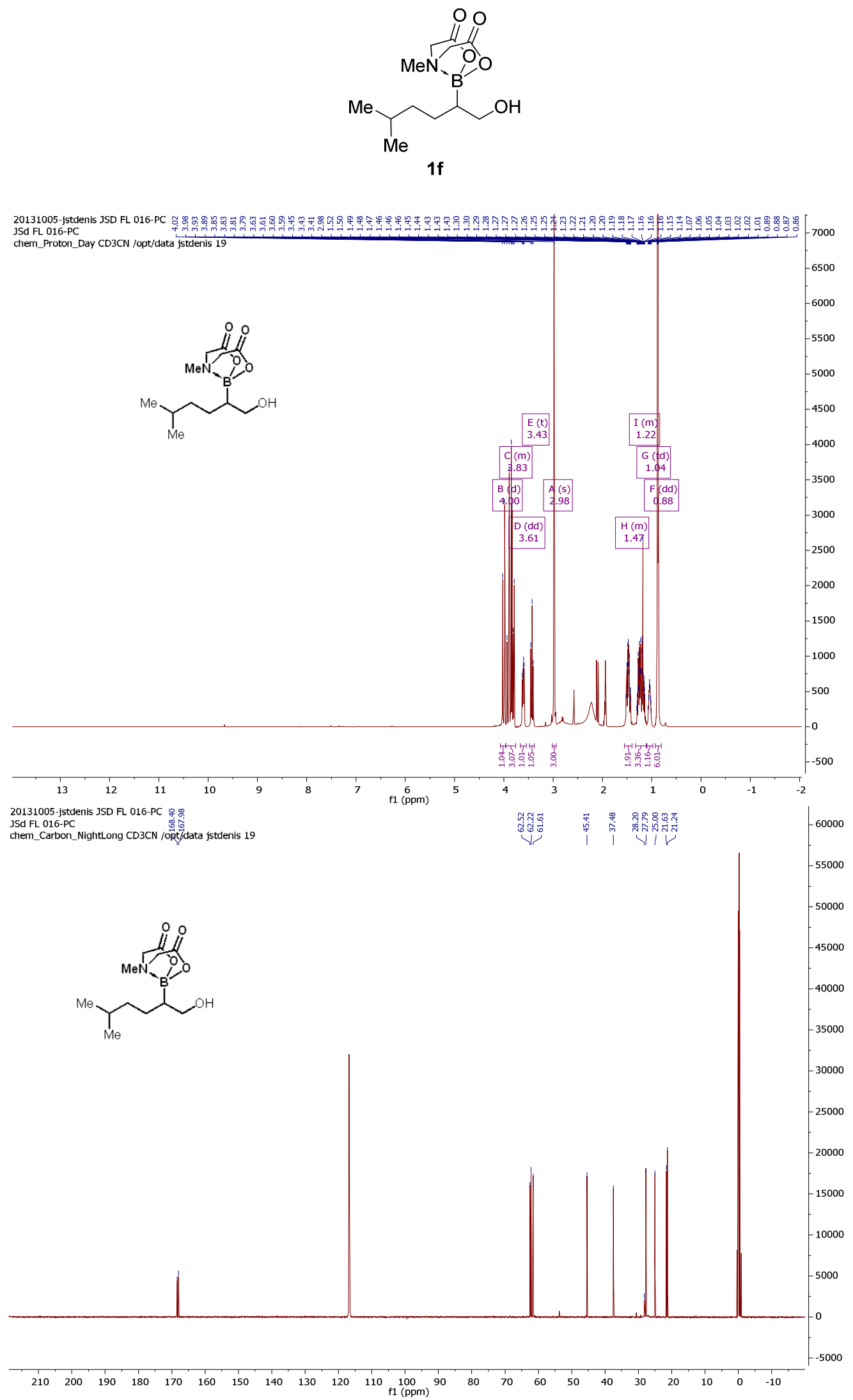




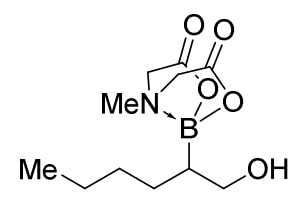

$1 g$

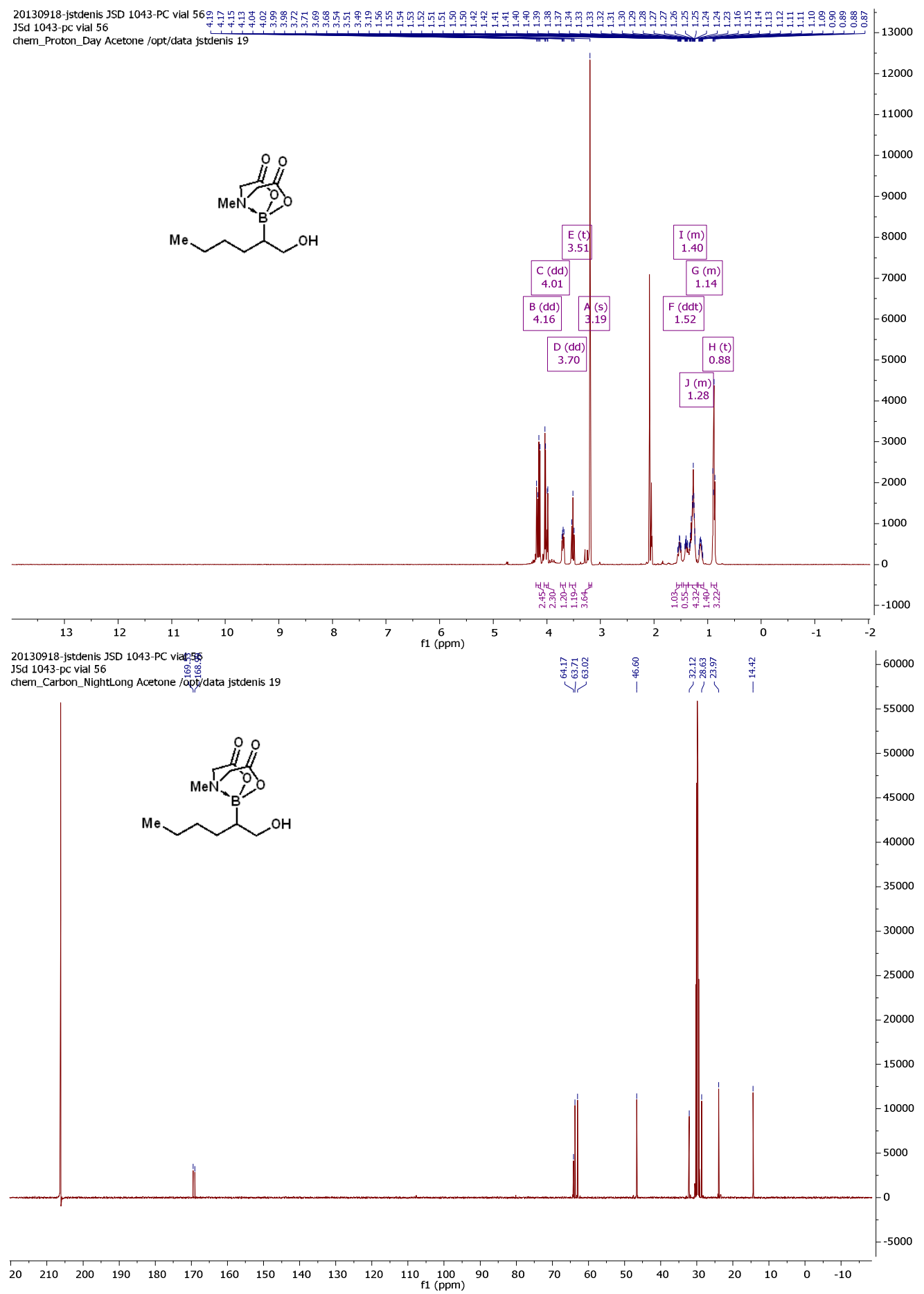




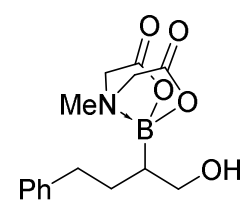

1h

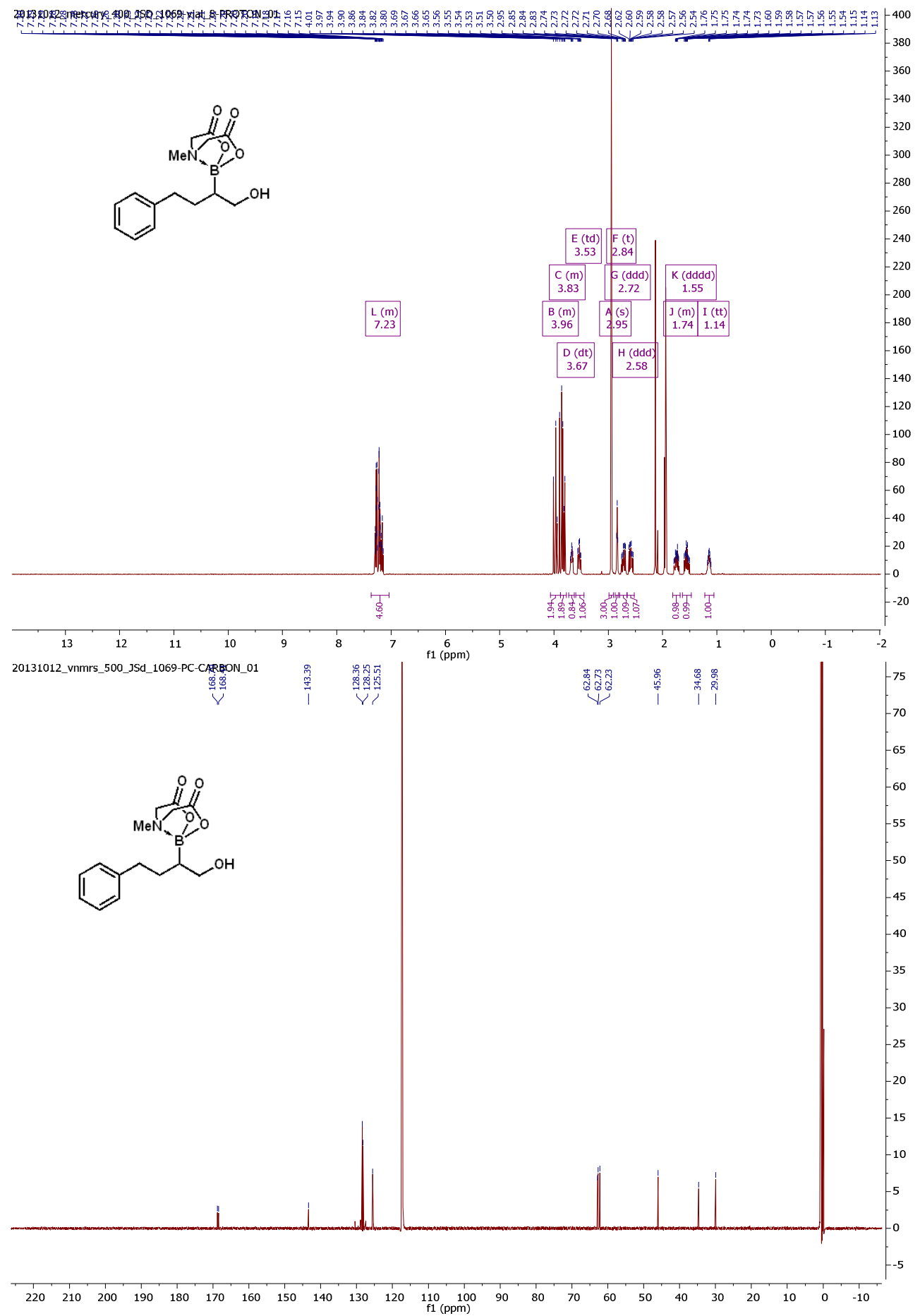




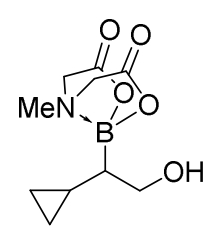

$1 \mathrm{i}$

20140205-jtdenis JSd 1181-PC viakl 36

chem_Proton_Day CD3CN/opt/data jstdenis 57

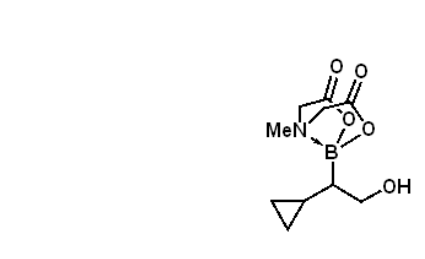

20140205-jstdenis JSd 1181-PC viafl Is 56
JSD 1181-PC vial 36

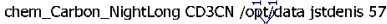
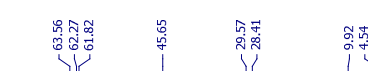

0000

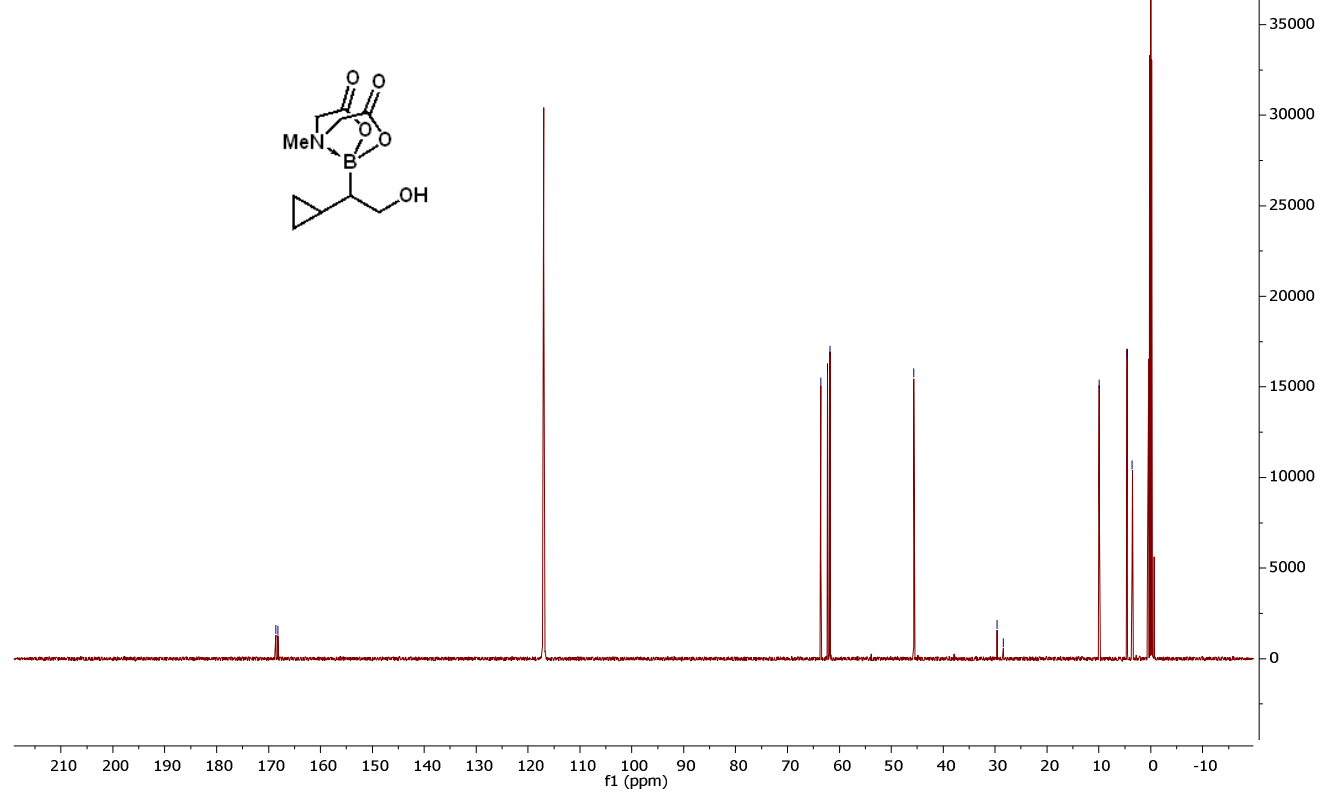




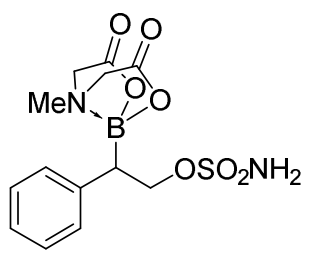

$2 a$

20130204-jstdenis JSD 792-PC.1.fic

chem_Proton_Day CDZCN/opt/data jstdenis 41
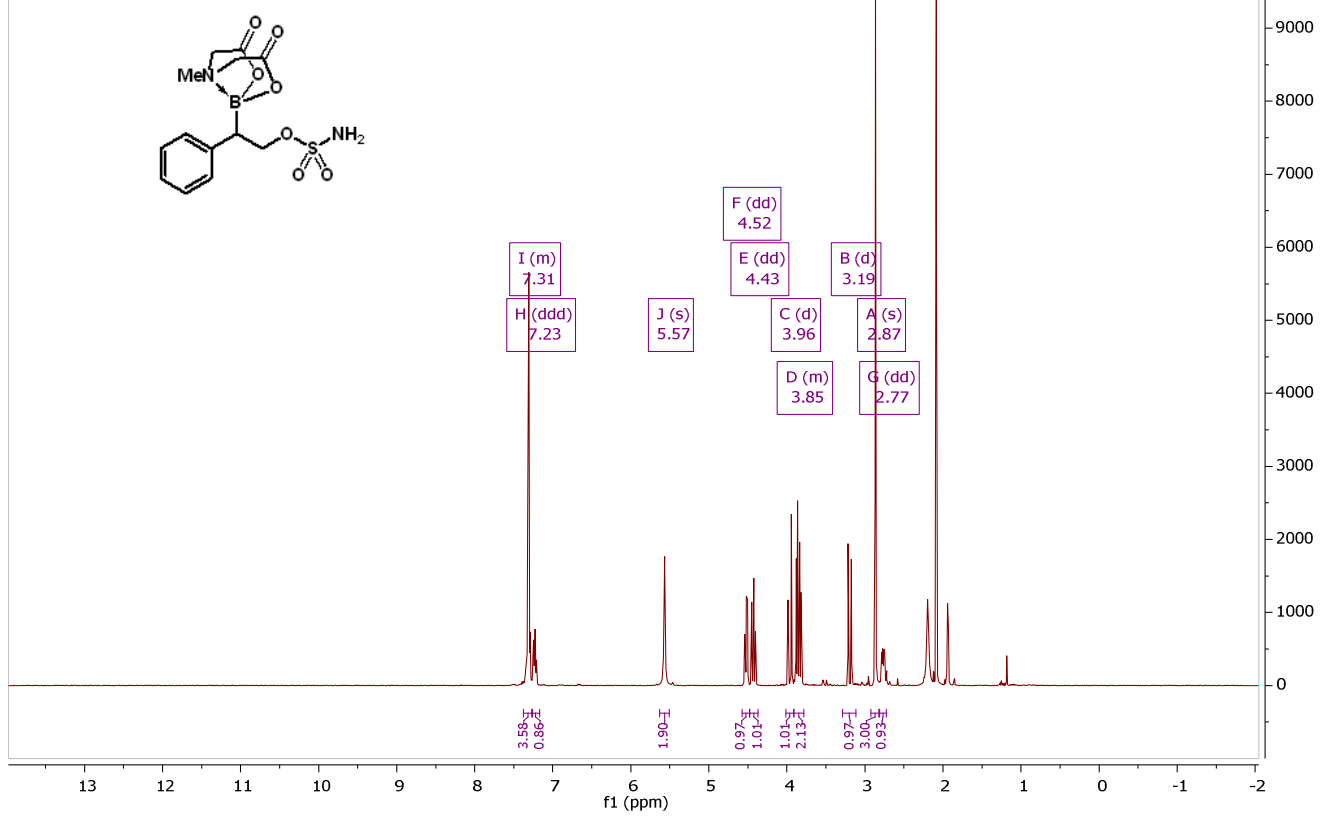

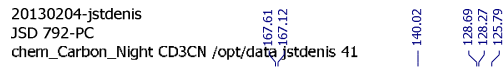

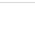




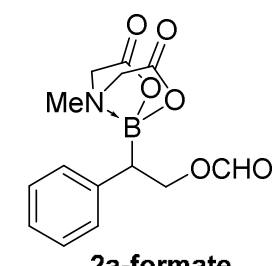

20131012_vnmrs_500_JSd_1064-formate-PROTON_01

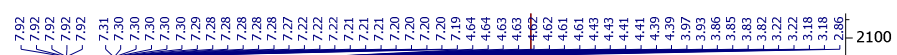
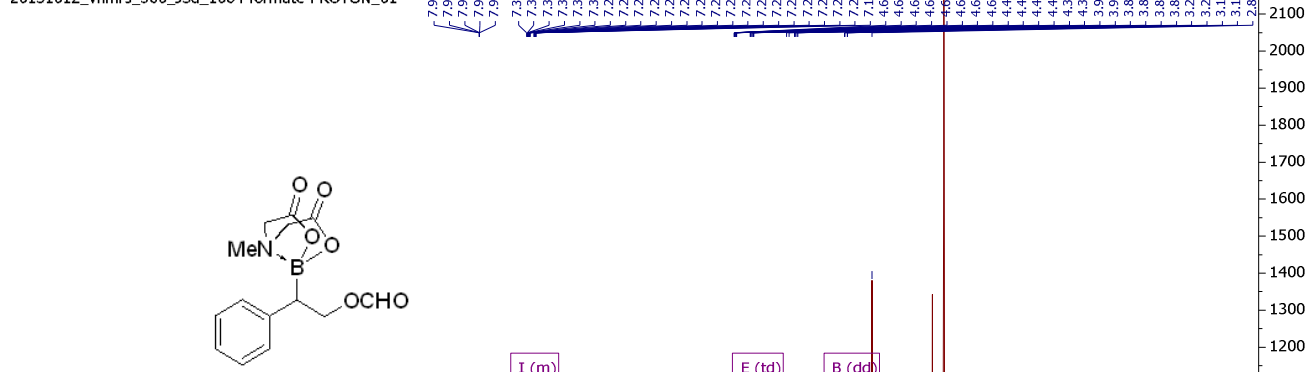

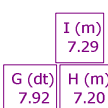

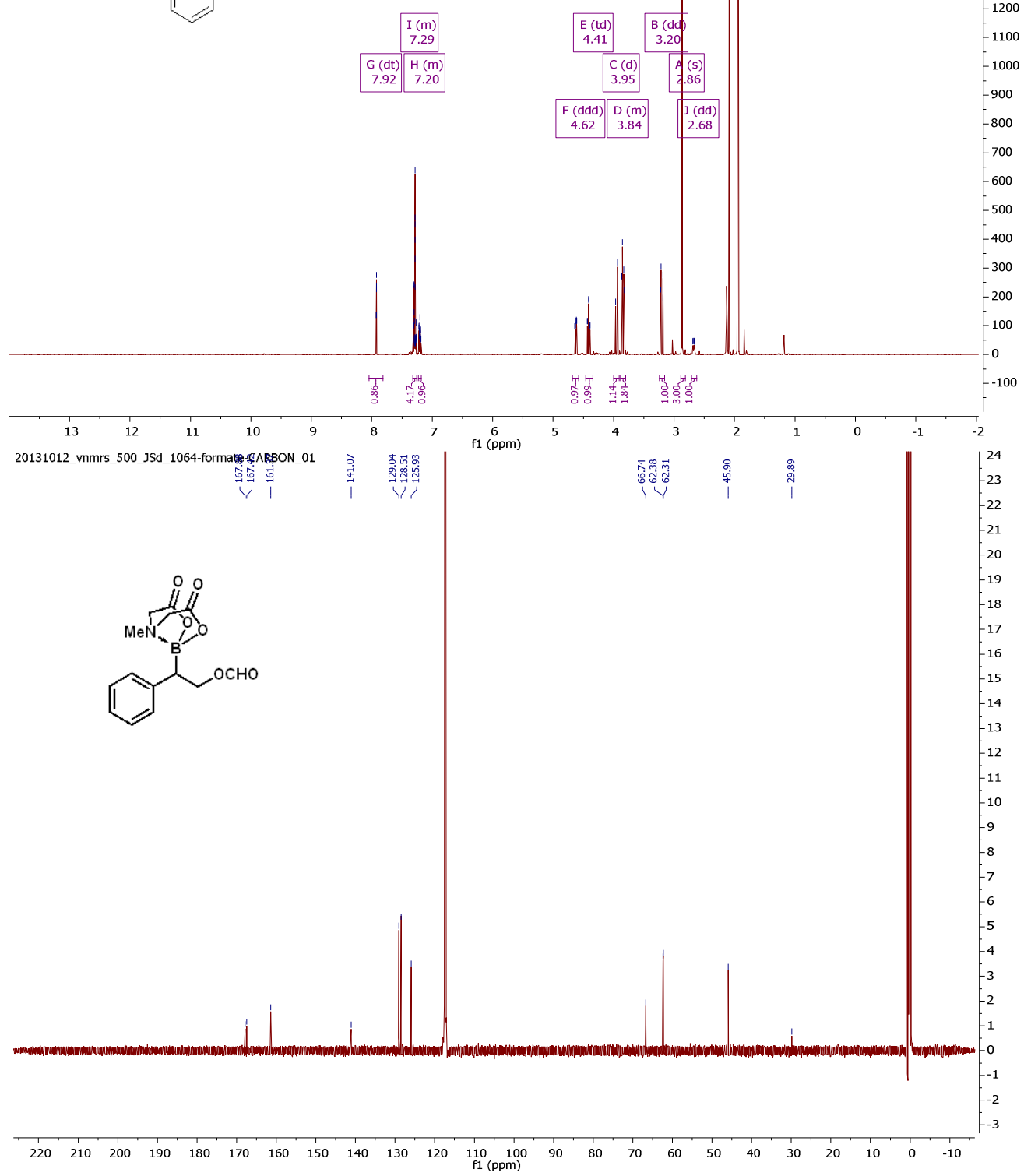

\section{2a-formate}




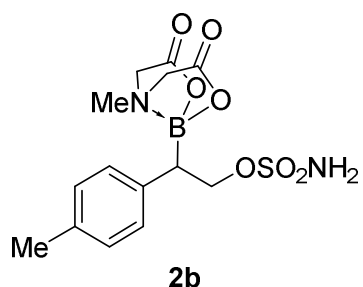

20131012-jstdenis JSd 1065-vial 47

JSD 1065-vial 47

chem_Proton_Day CD3CN /opt/data jstdenis 2

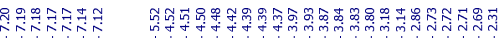

$-3000$

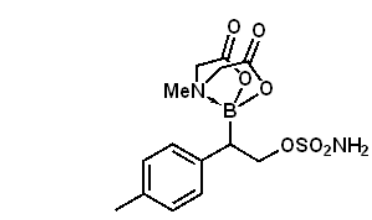

2800

2600

$-2200$

$-2000$

$-1800$

$-1600$

1400

$-1200$

1000

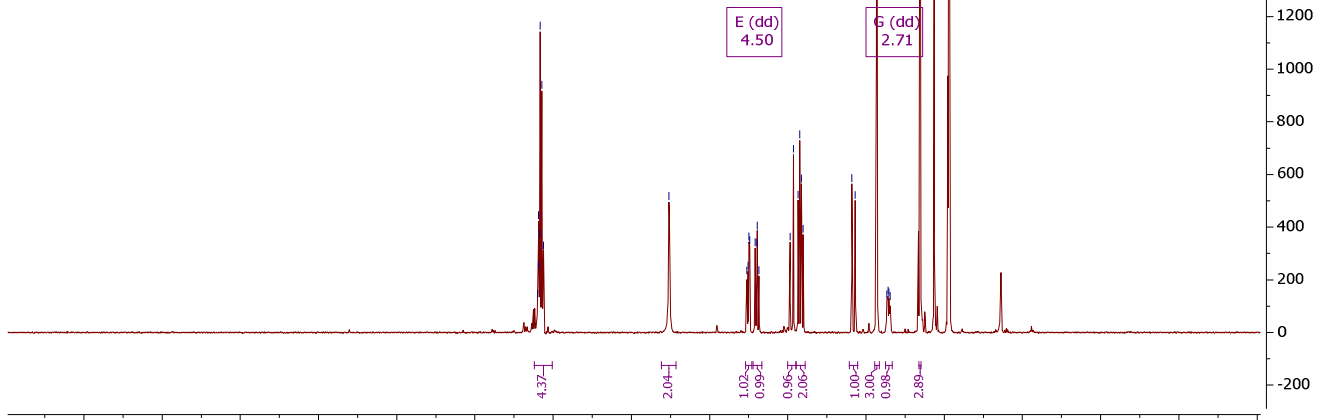

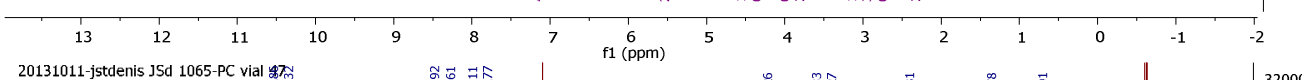

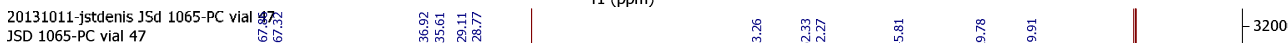

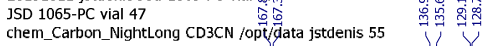
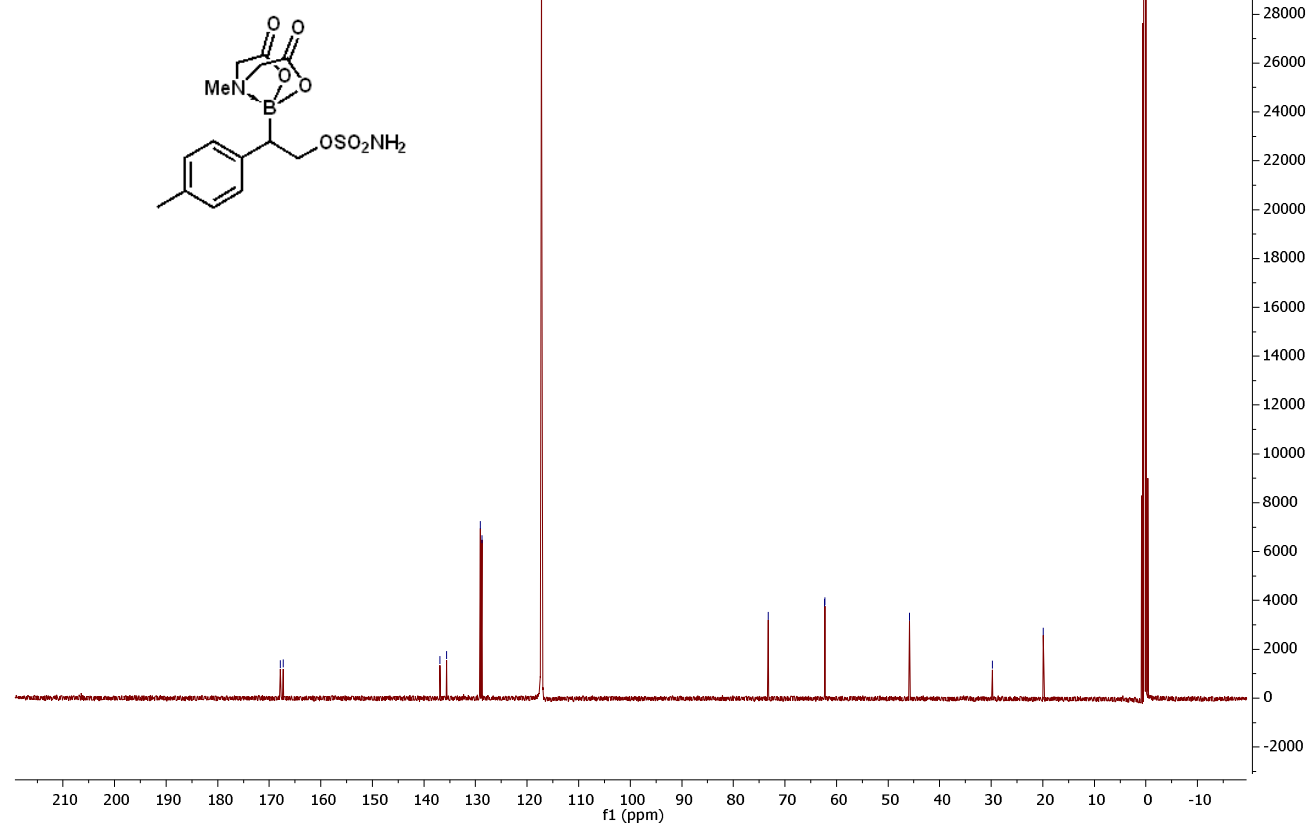


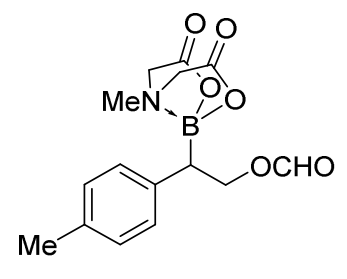

\section{2b-formate}

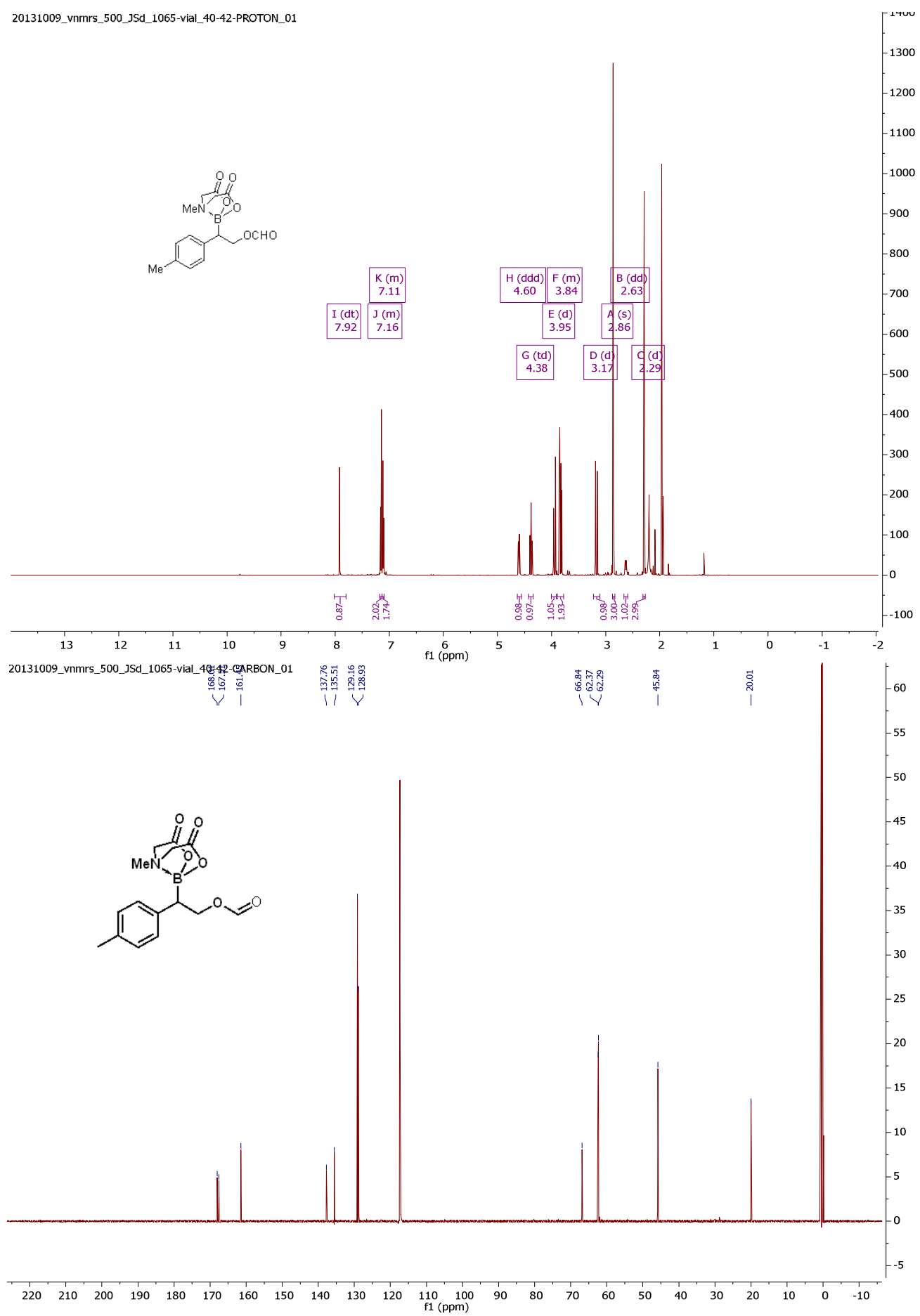



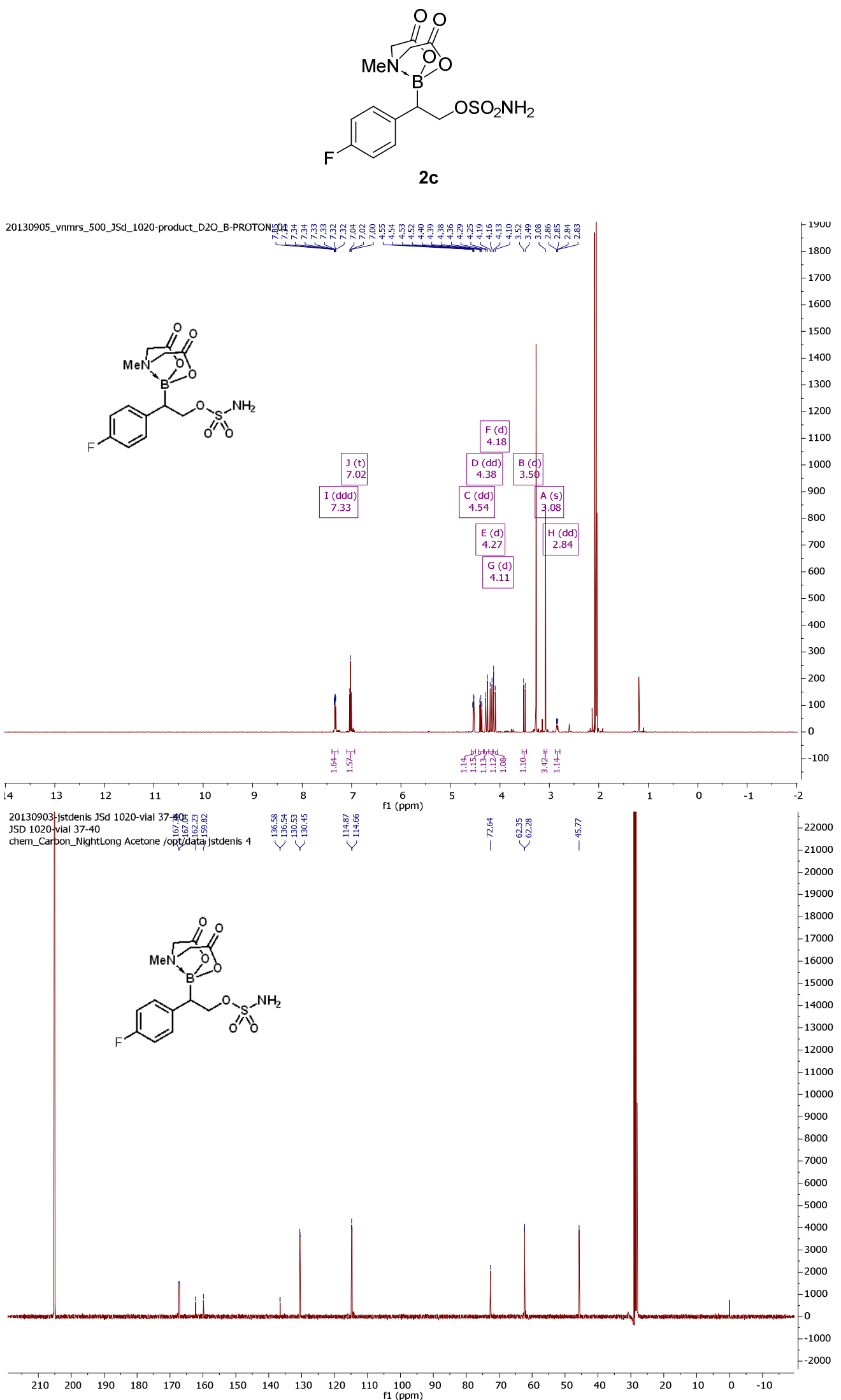


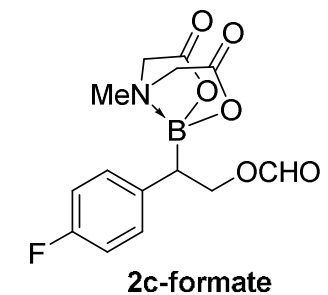

20130903--jtdenis JSD 1020-vial 33-36
JSD 1020-vial 33-36 JSD 1020-vial 33-36
chem_Proton_Day Acetone /opt/data jstdenis 3

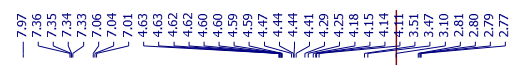
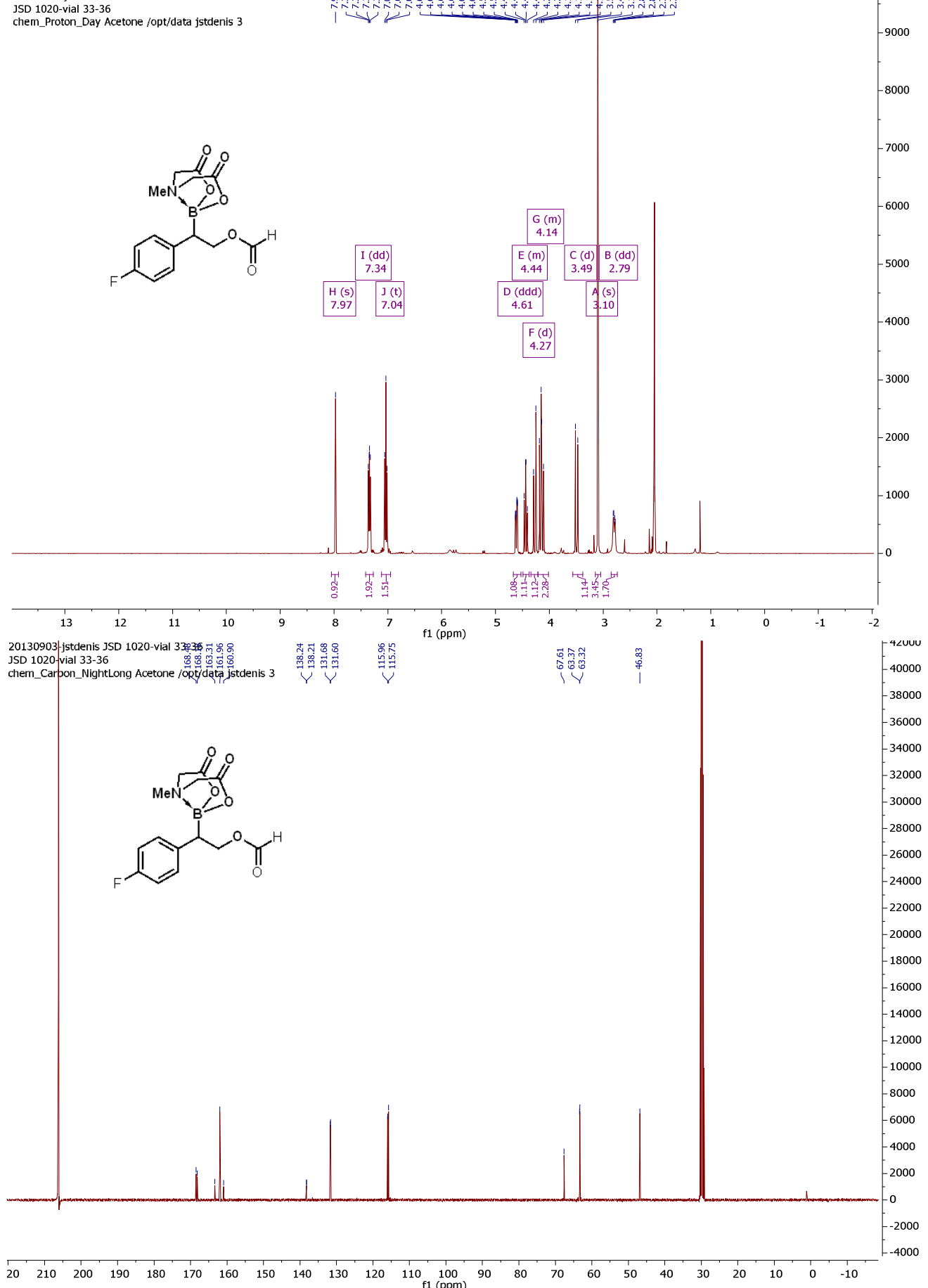


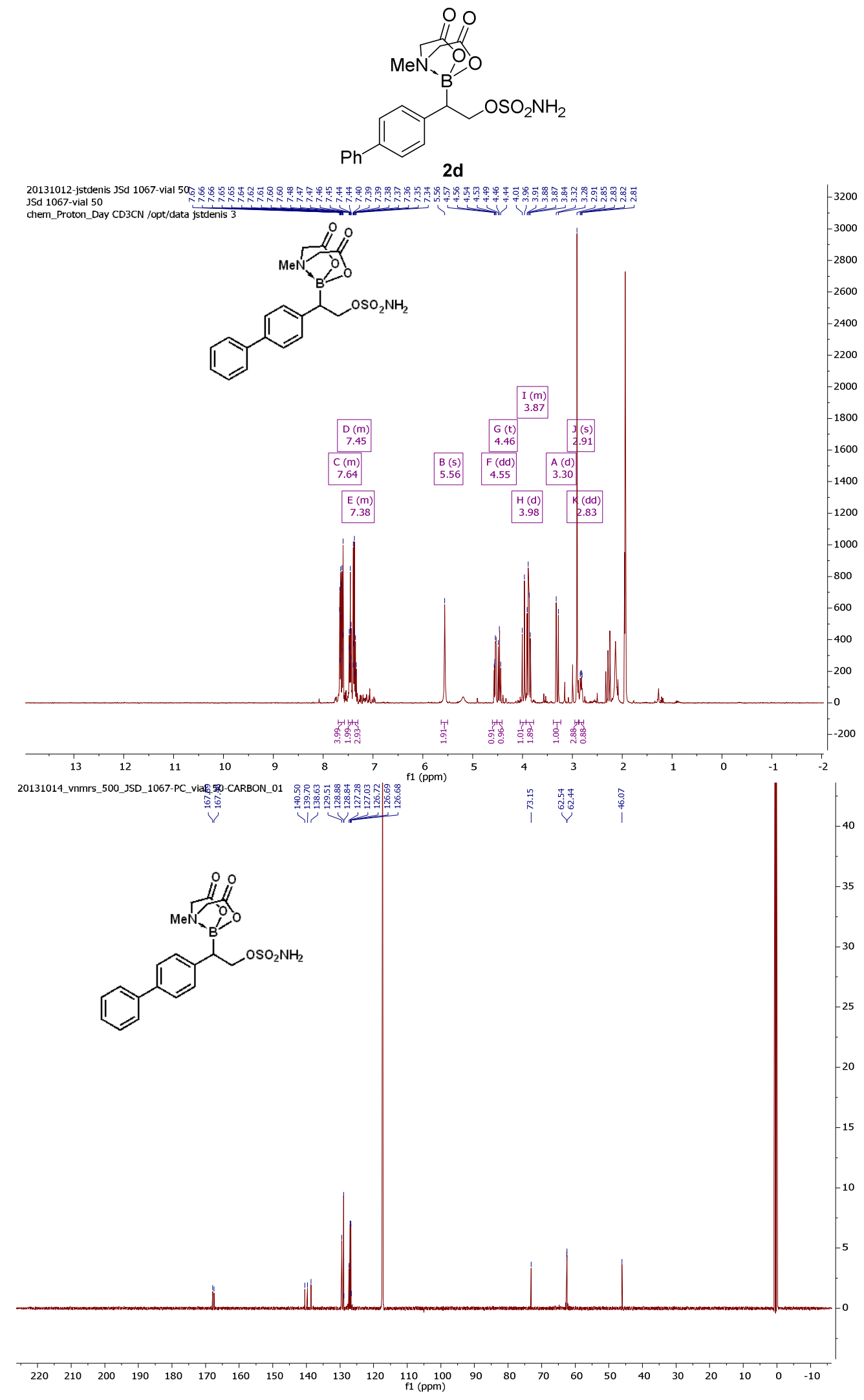




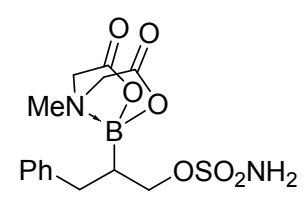

$2 e$

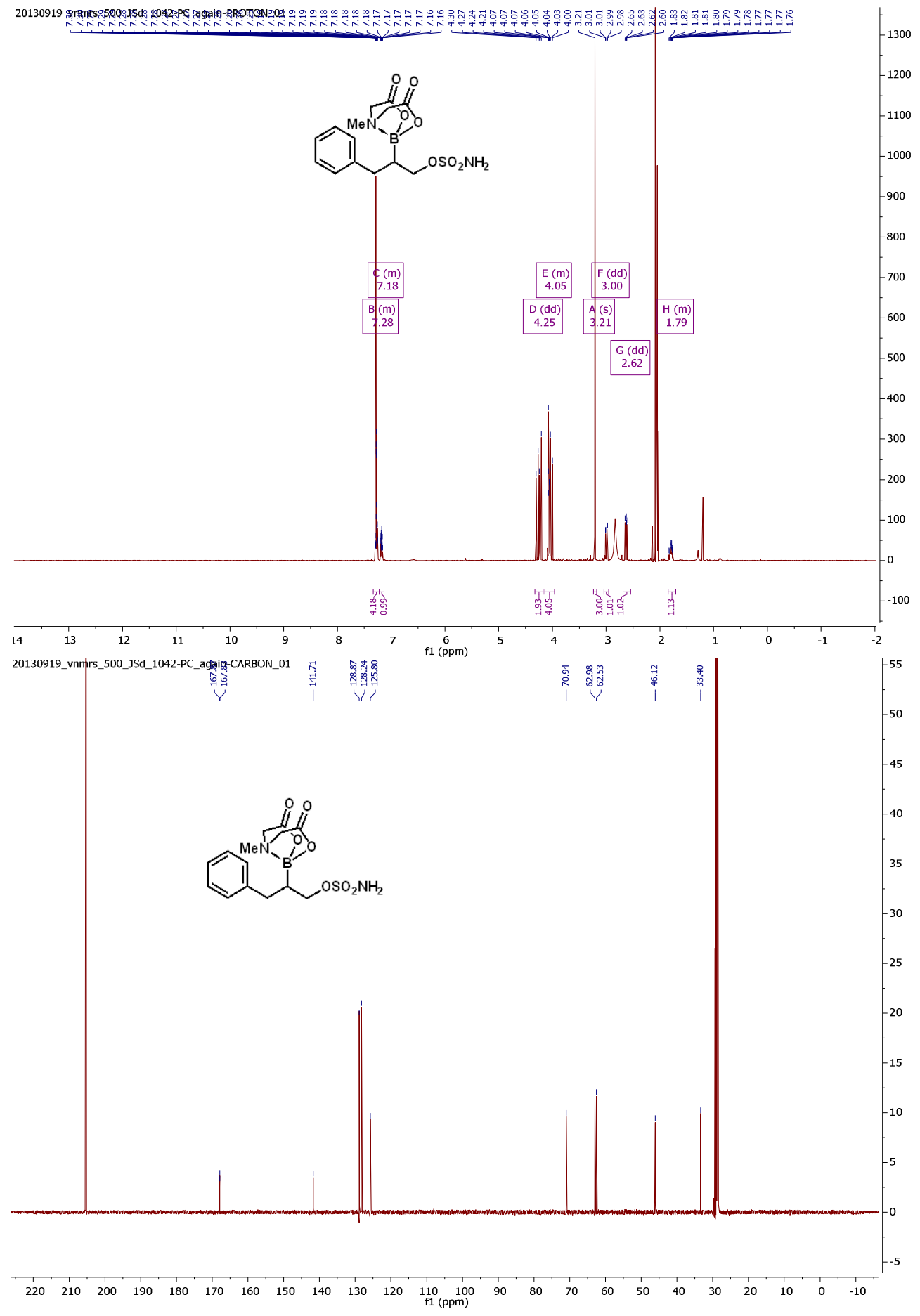



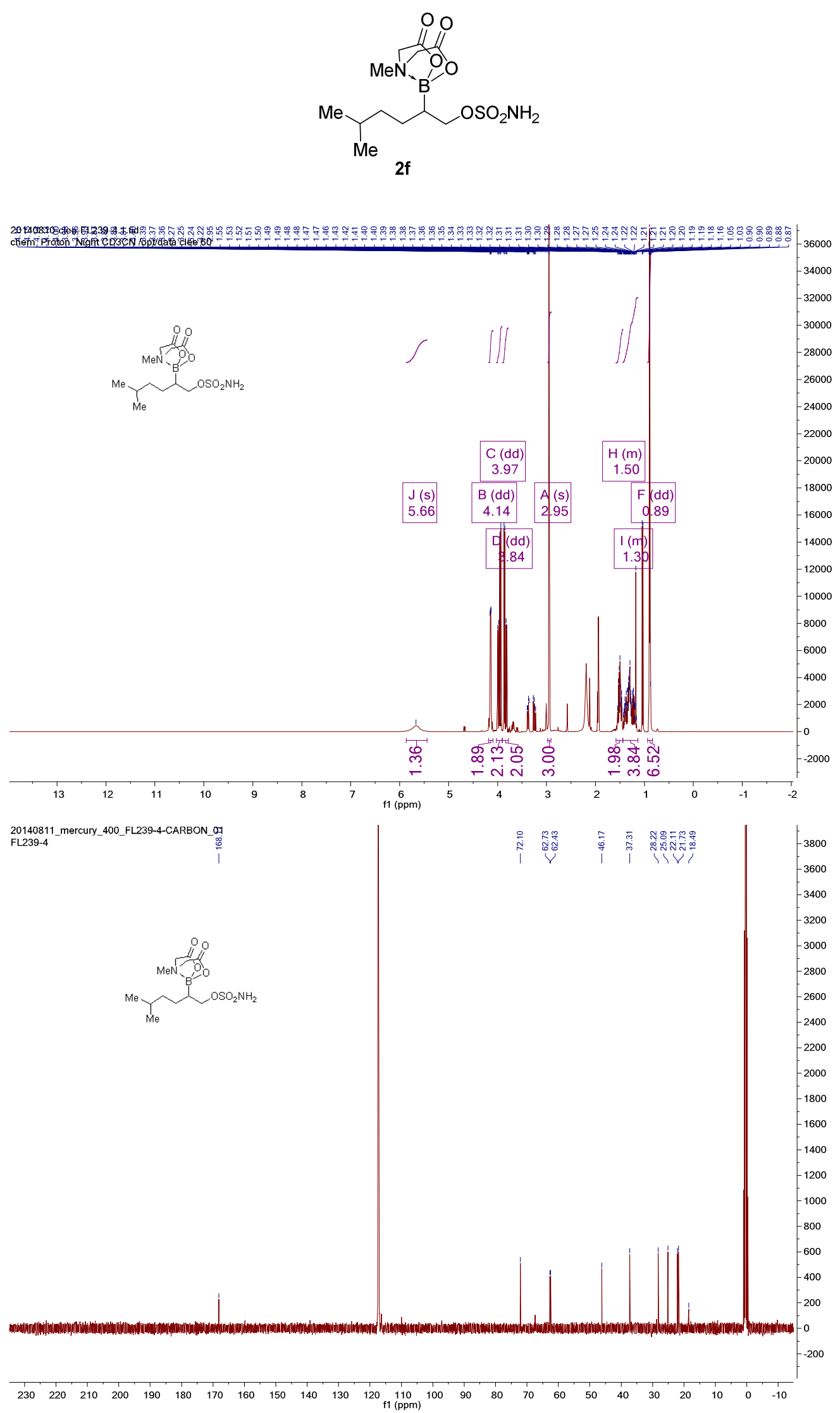


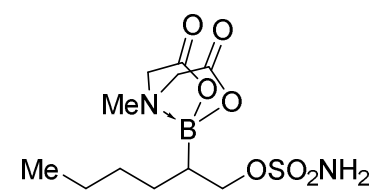

$2 \mathrm{~g}$

20130923-jstdenis JSd 1045-Pc vial 15 chem_Proton_Day Acetone /opt/data jstdenis 29
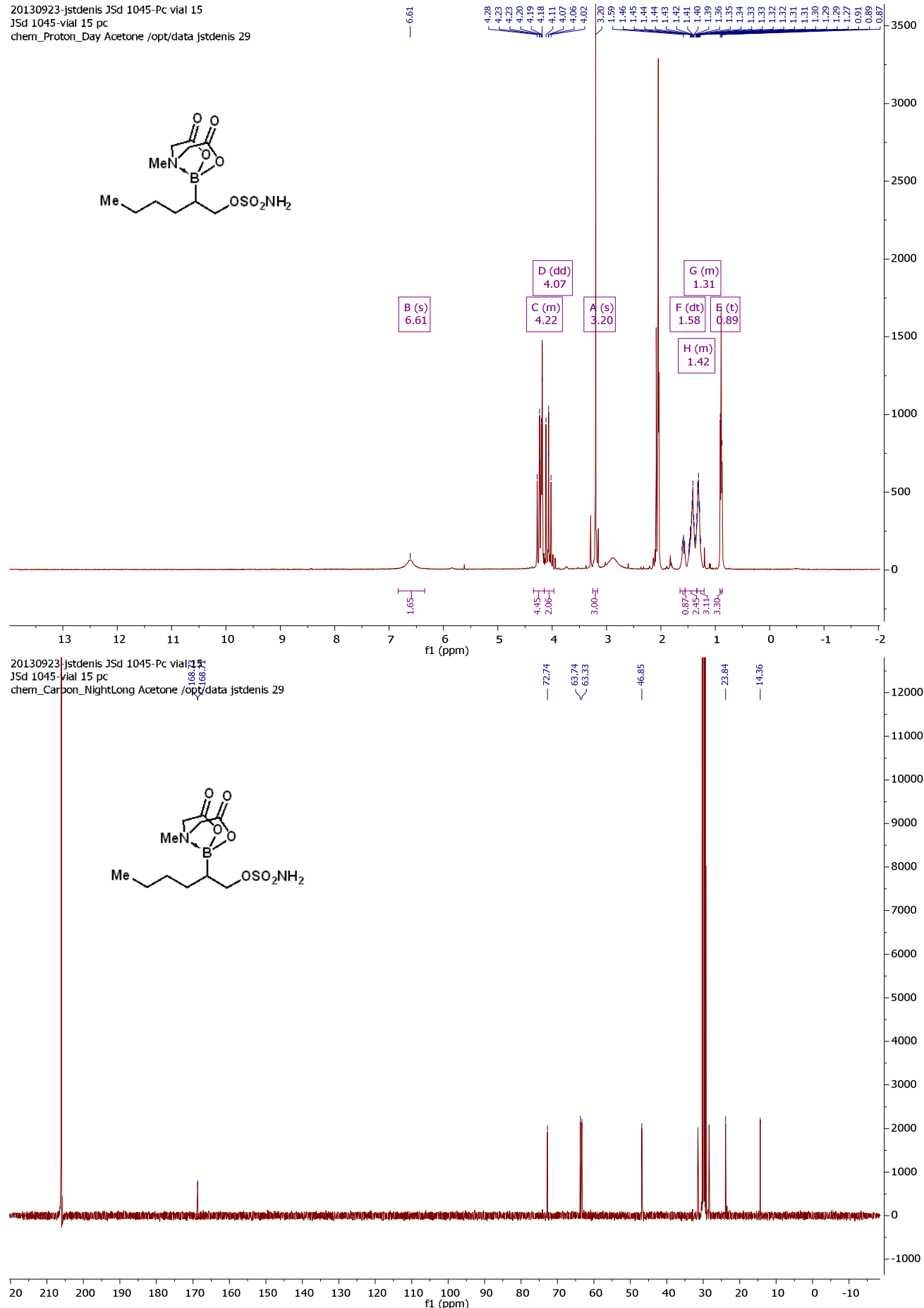


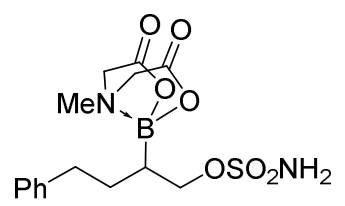

2h

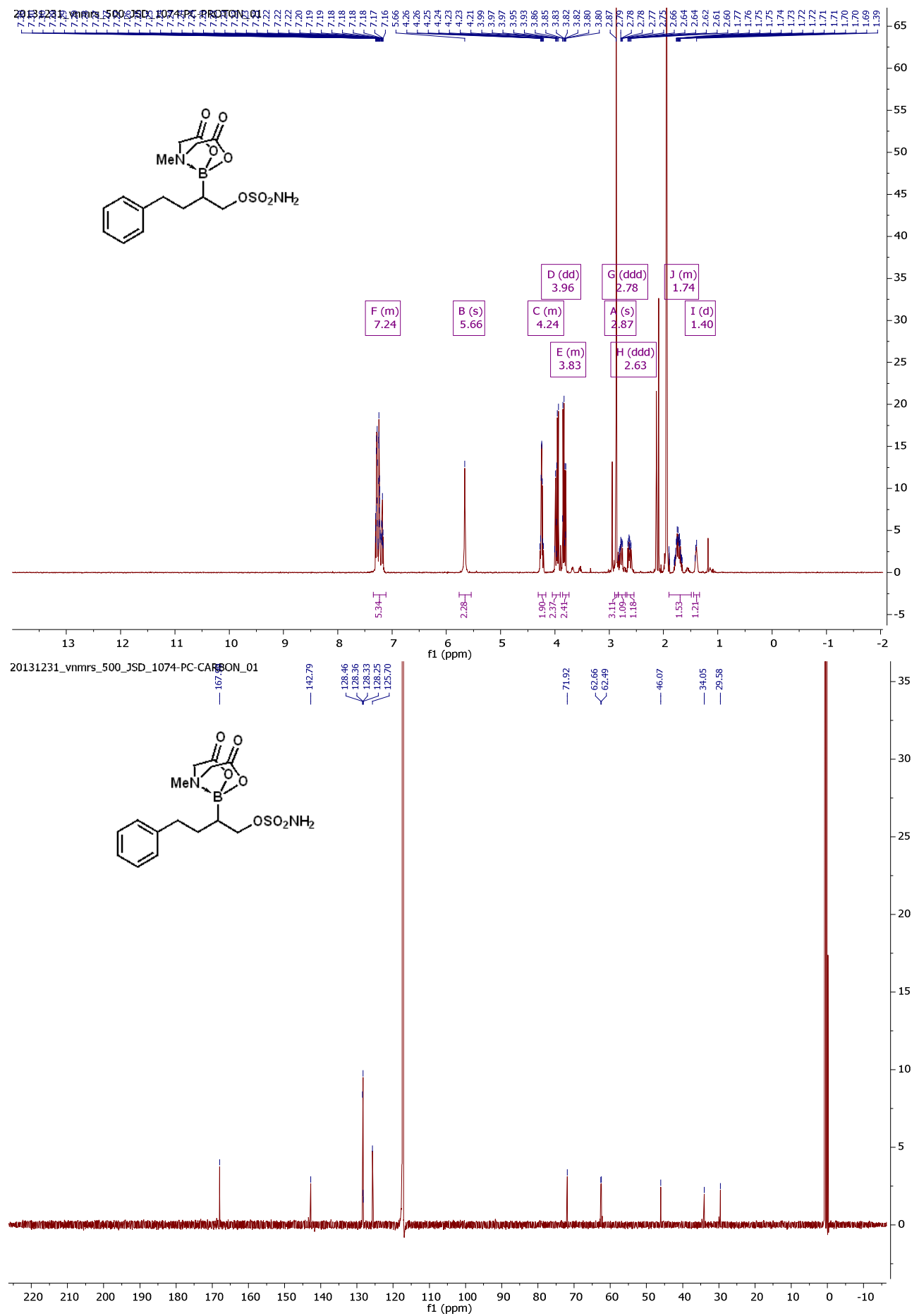




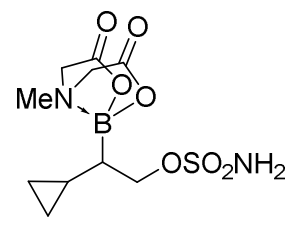

2i

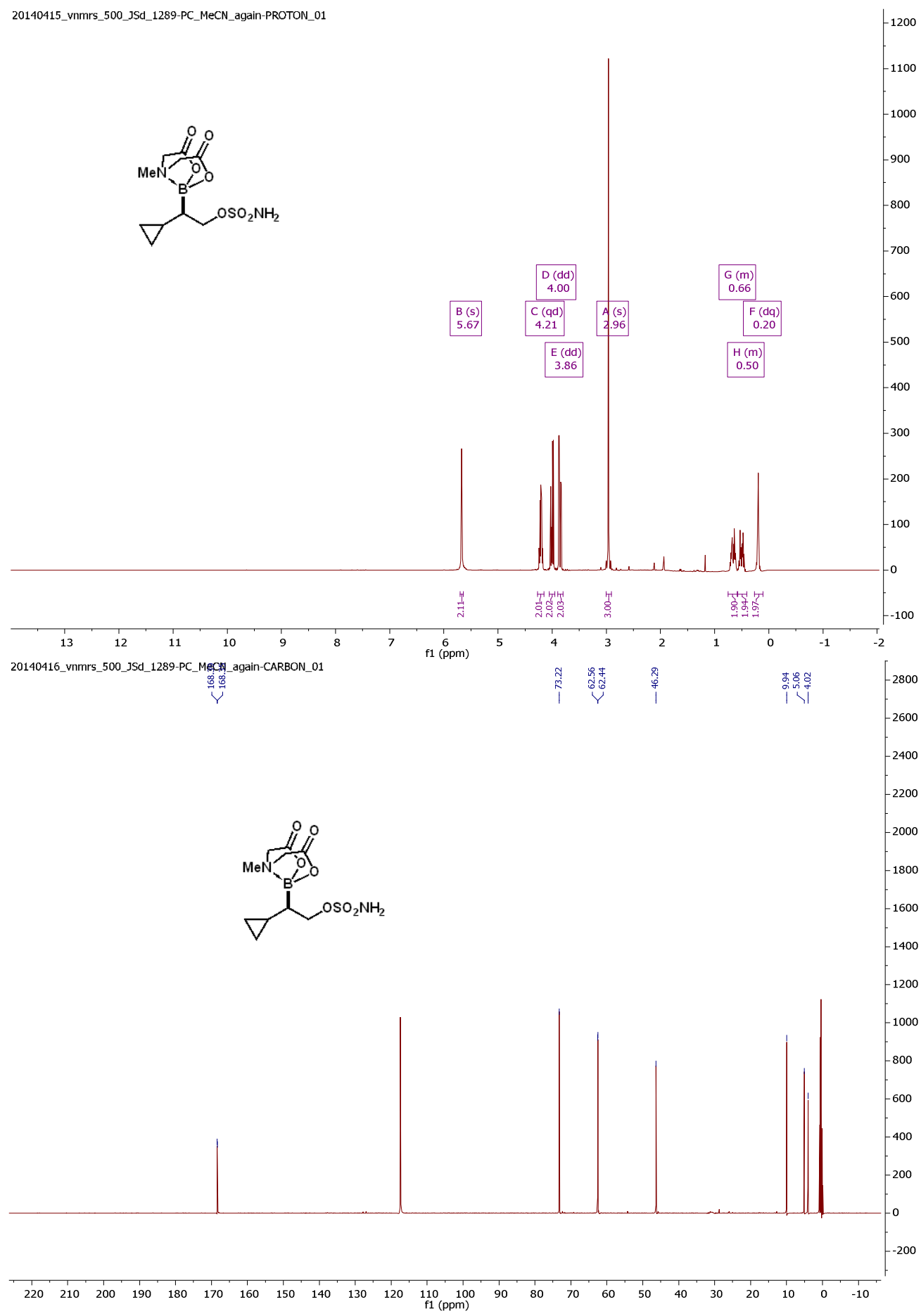




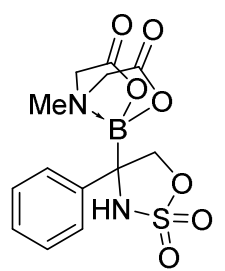

$3 a$

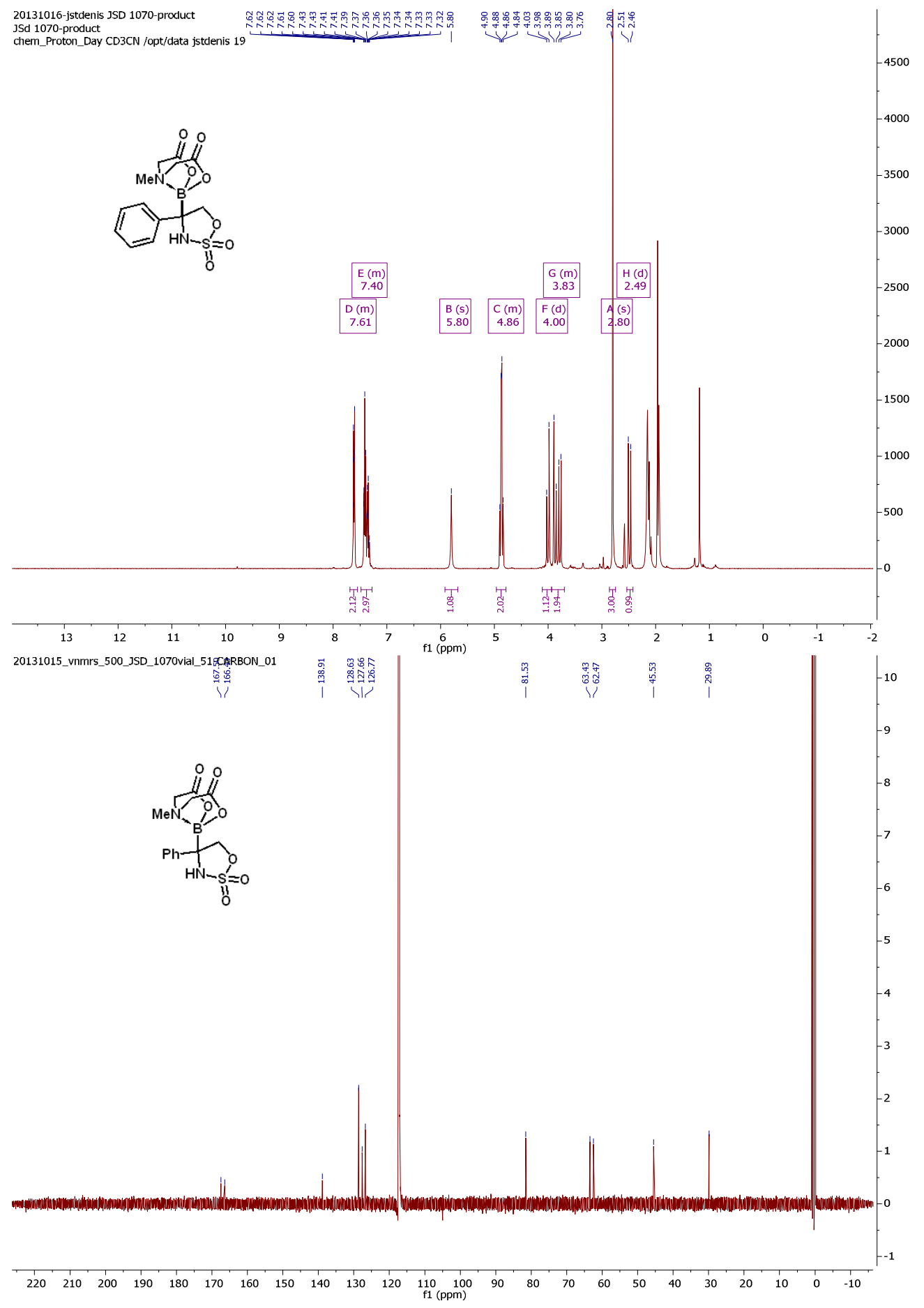




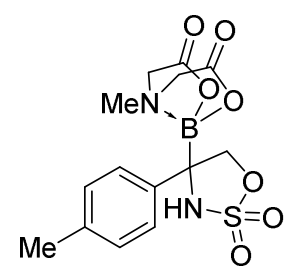

$3 b$

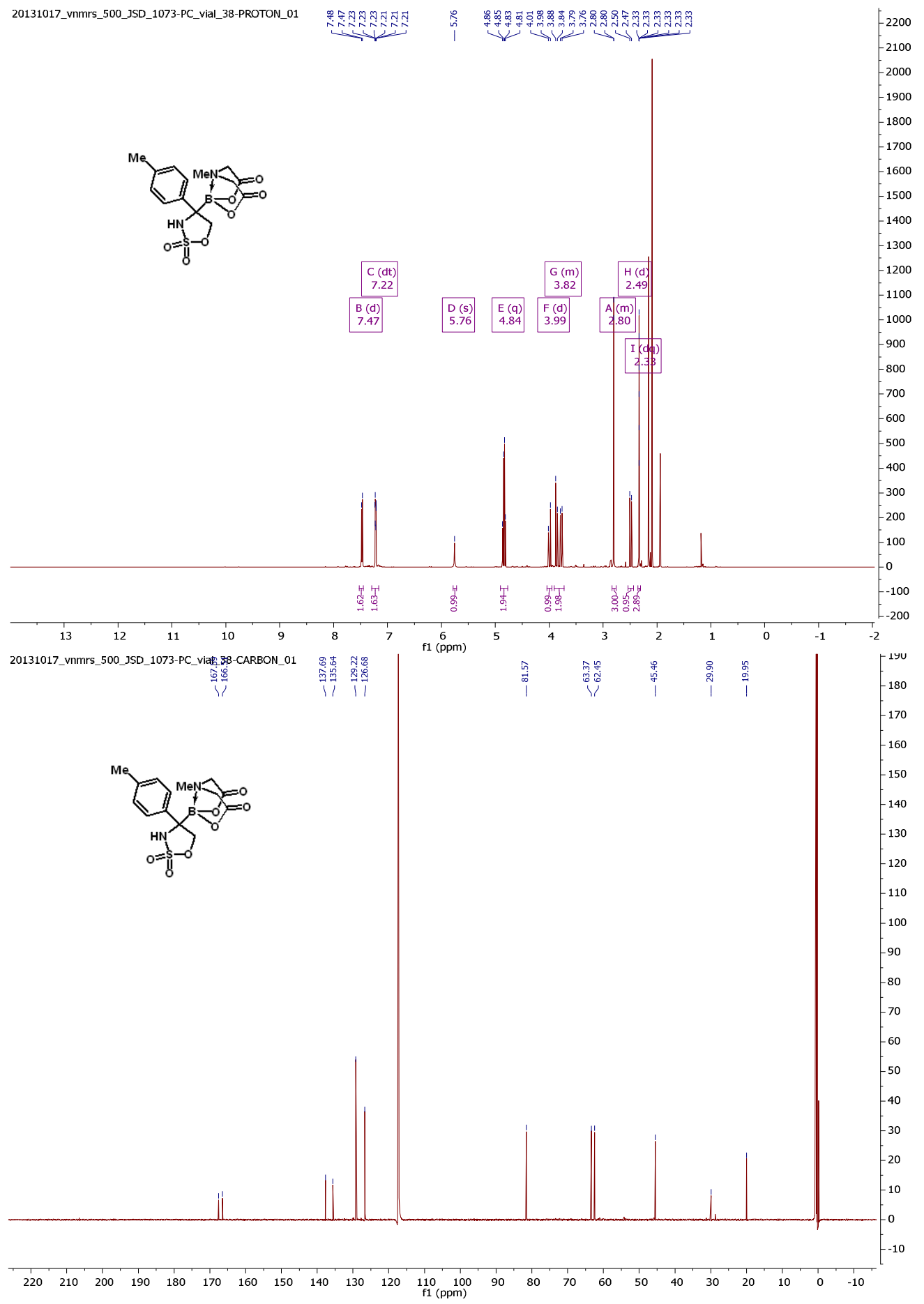




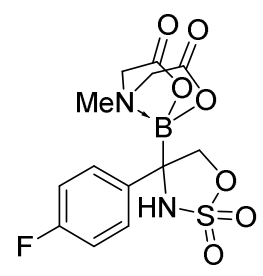

$3 c$

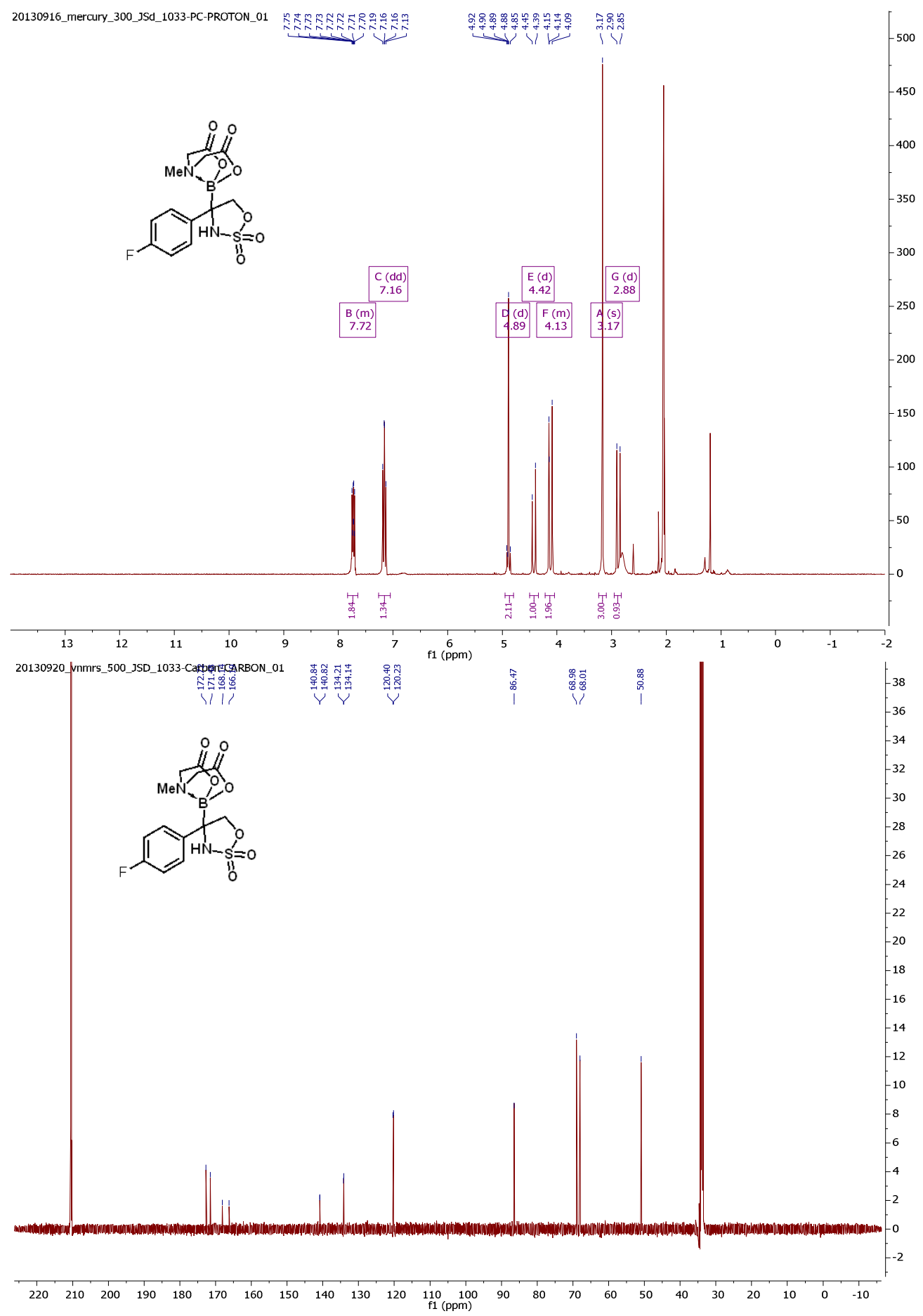




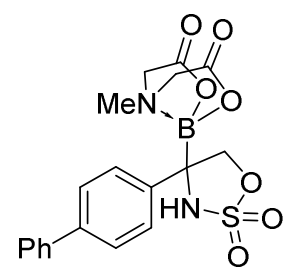

3d

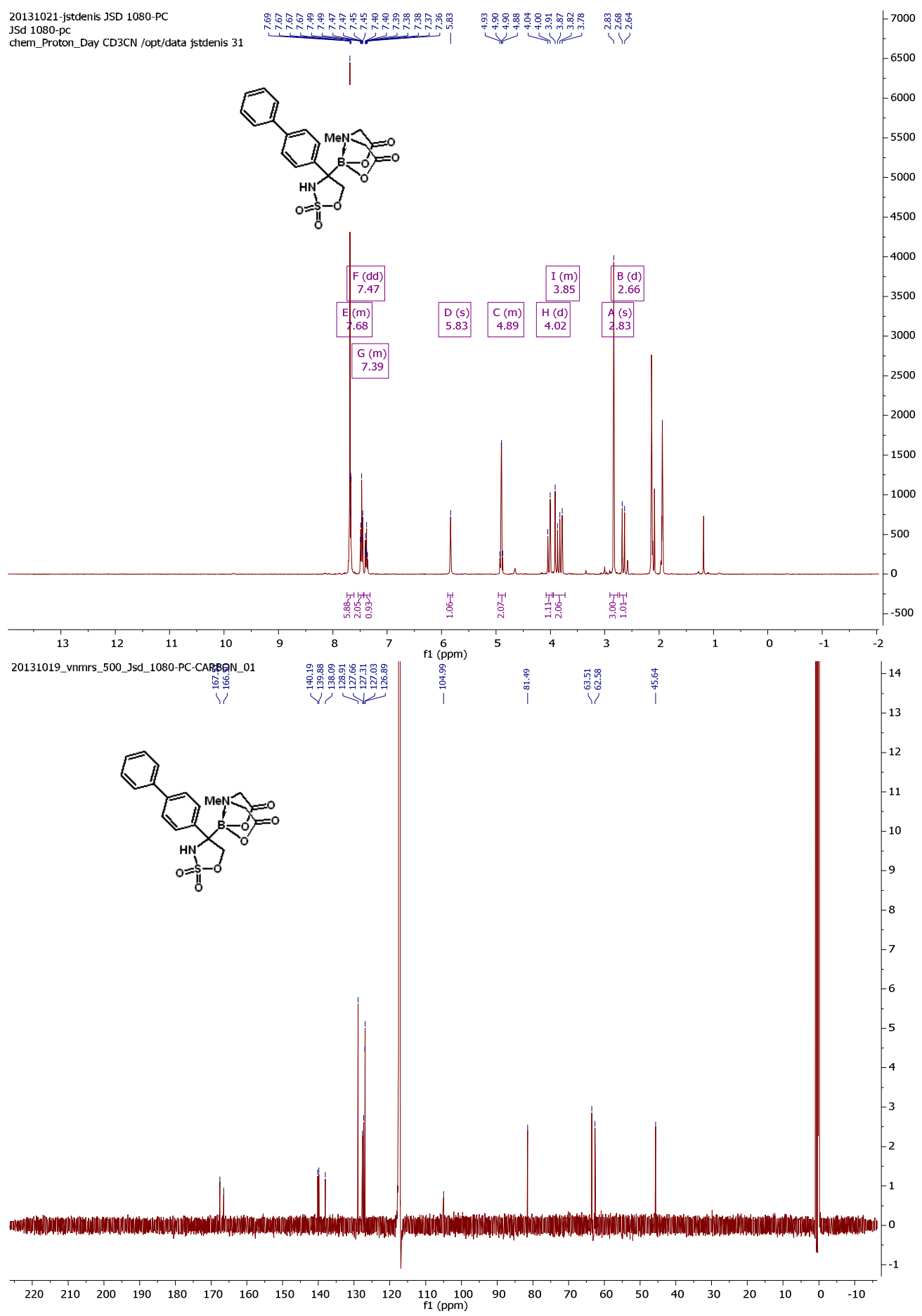



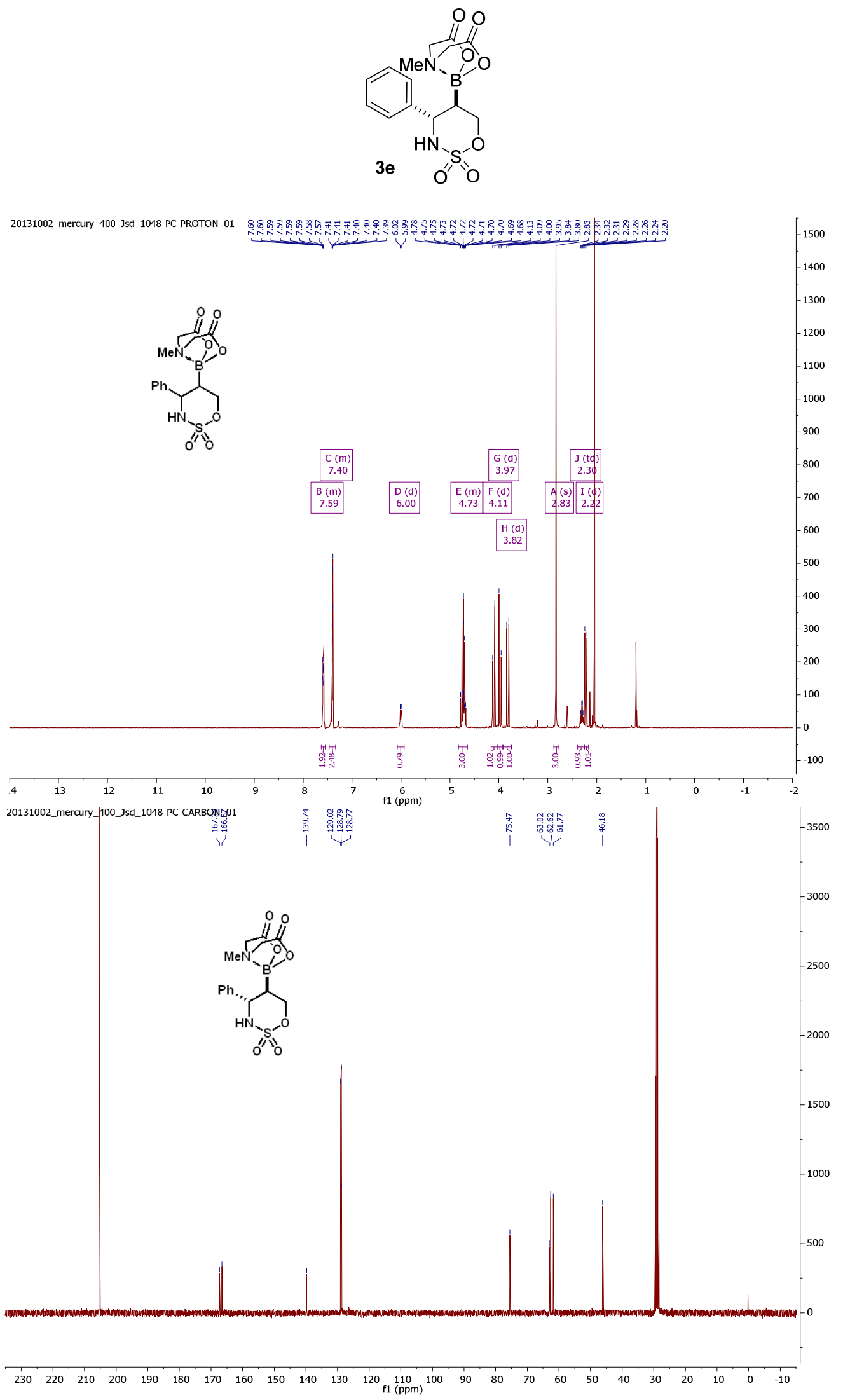


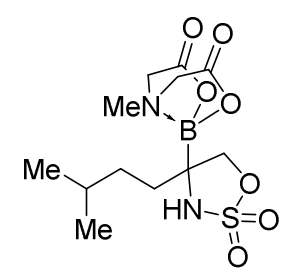

3f'

20131106-jstdenis JSA FL 041-3 -5 membered ring JSd FL O41-3 5 membered ring
chem_Proton_Day CD3CN /opt/data jstdenis 57

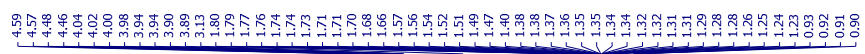
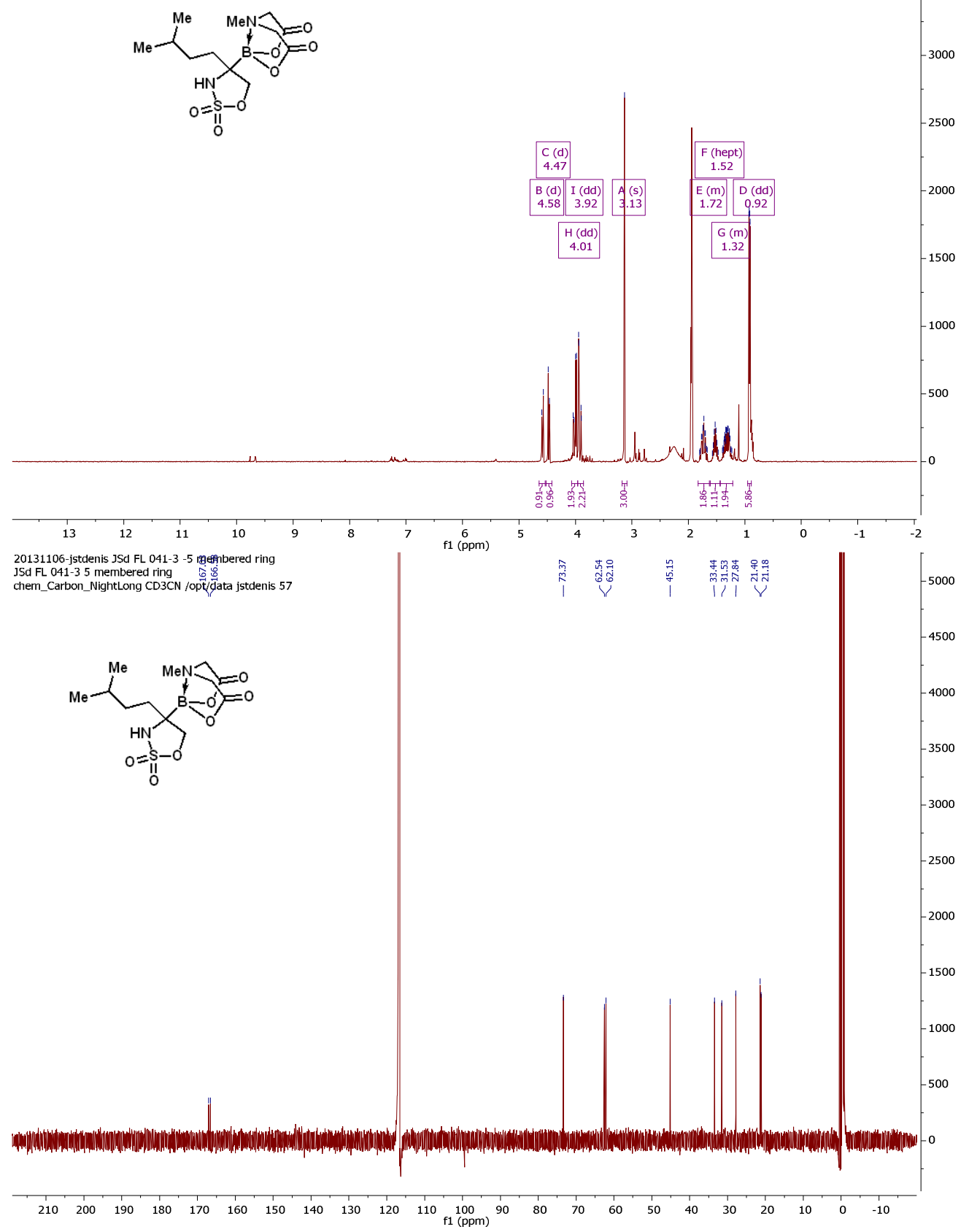


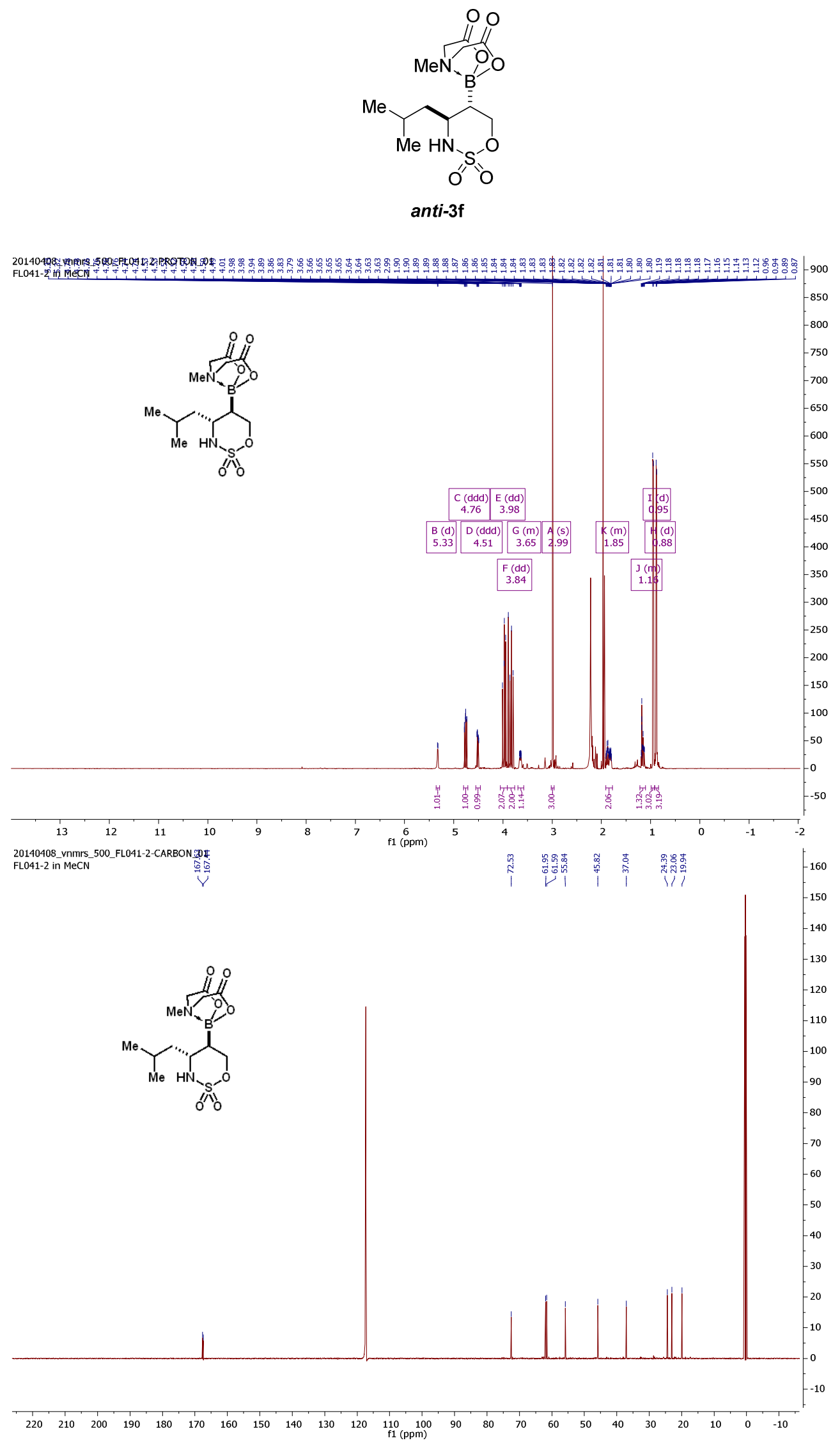




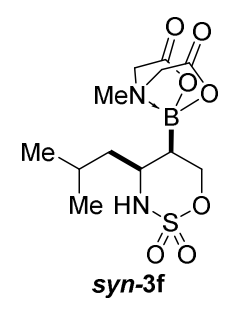

20140408_vnmrs_500_FL041-1-PROTON_01 FL041-1 in MeCN

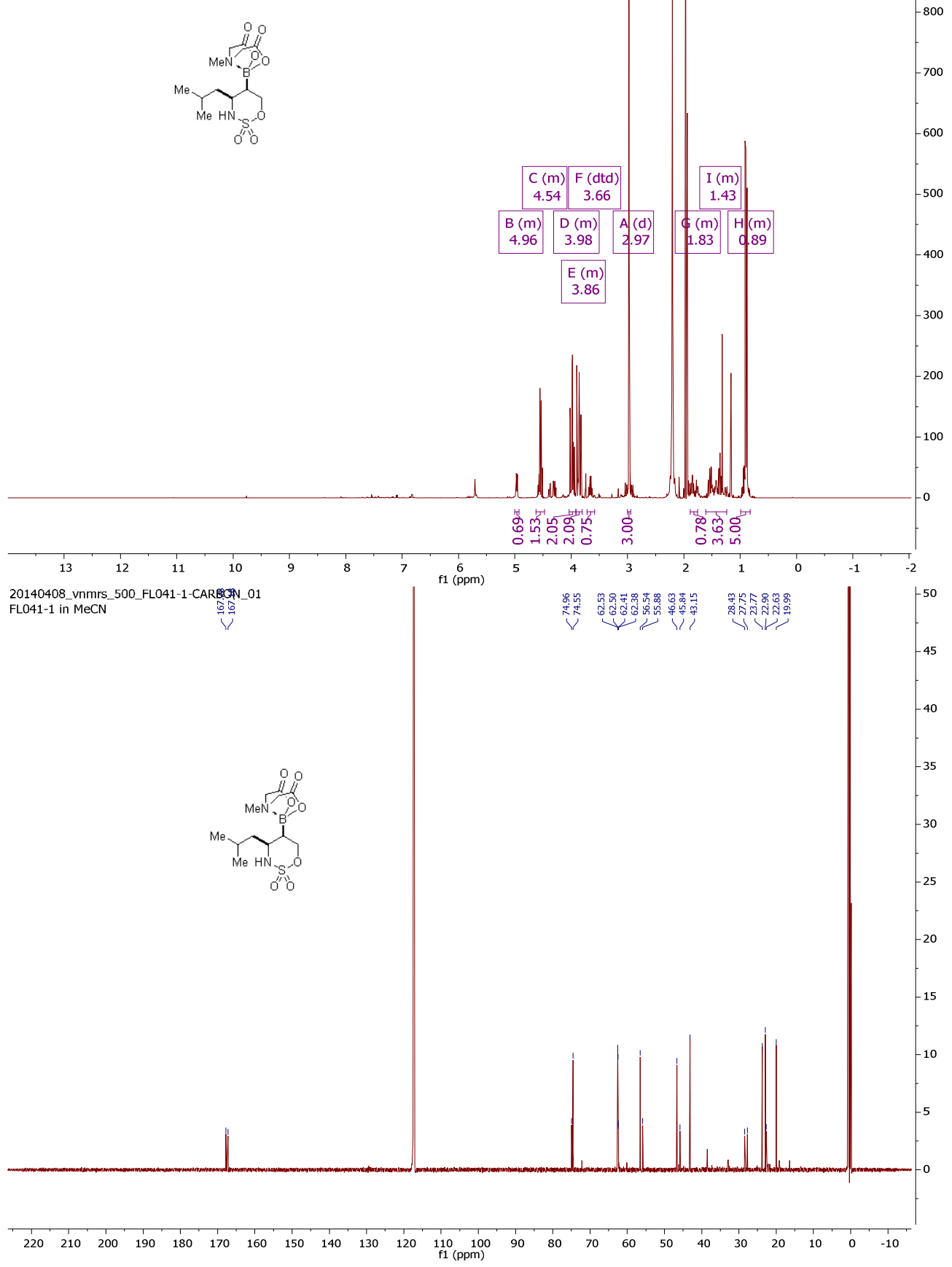




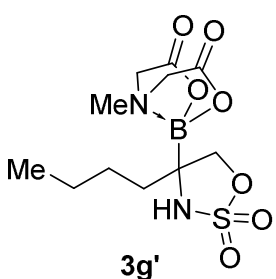

20131120-clee-FL045-1-MeCN chem_Proton_Day CD3CN /opt/data clee 5

诲
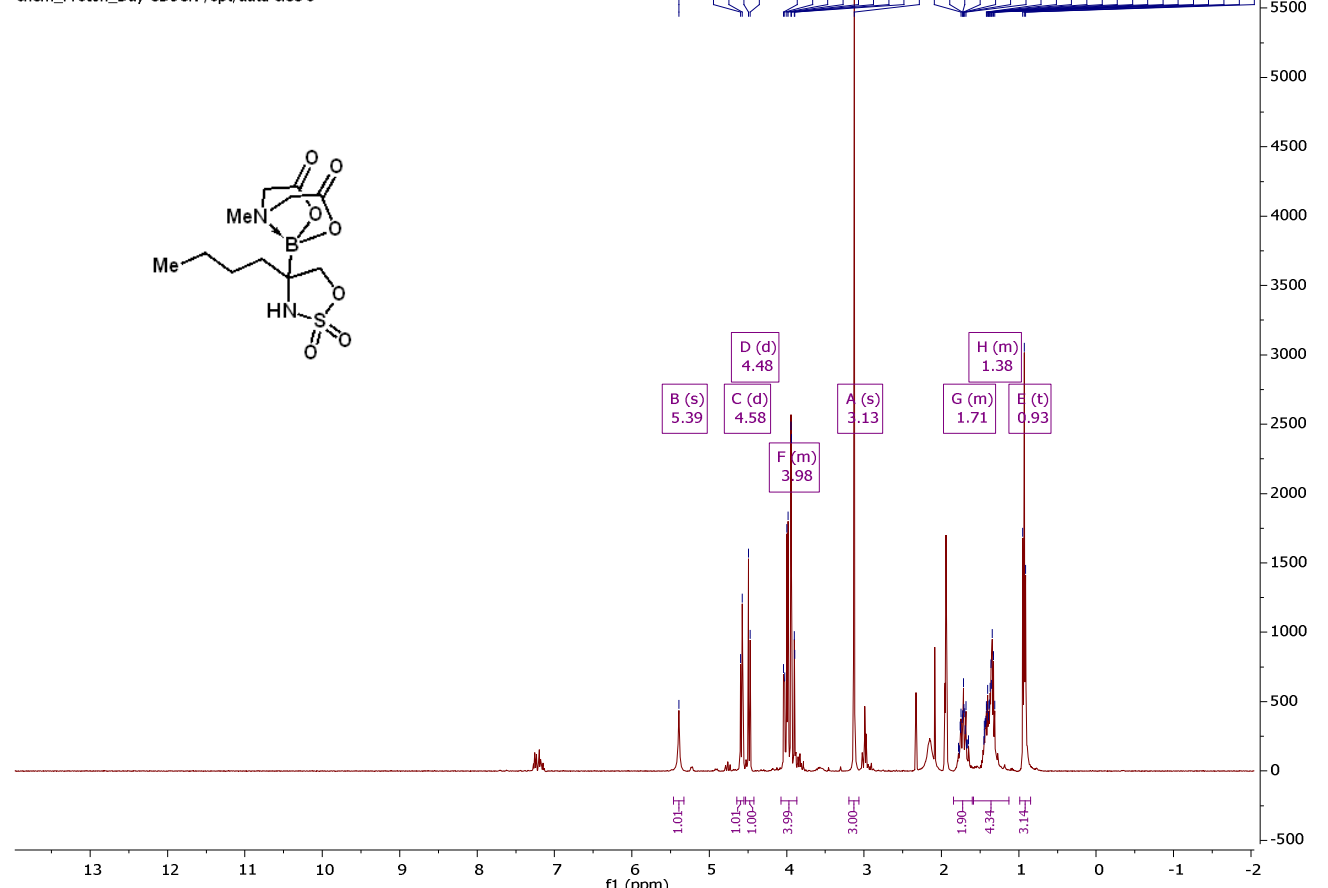

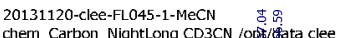
$\checkmark$
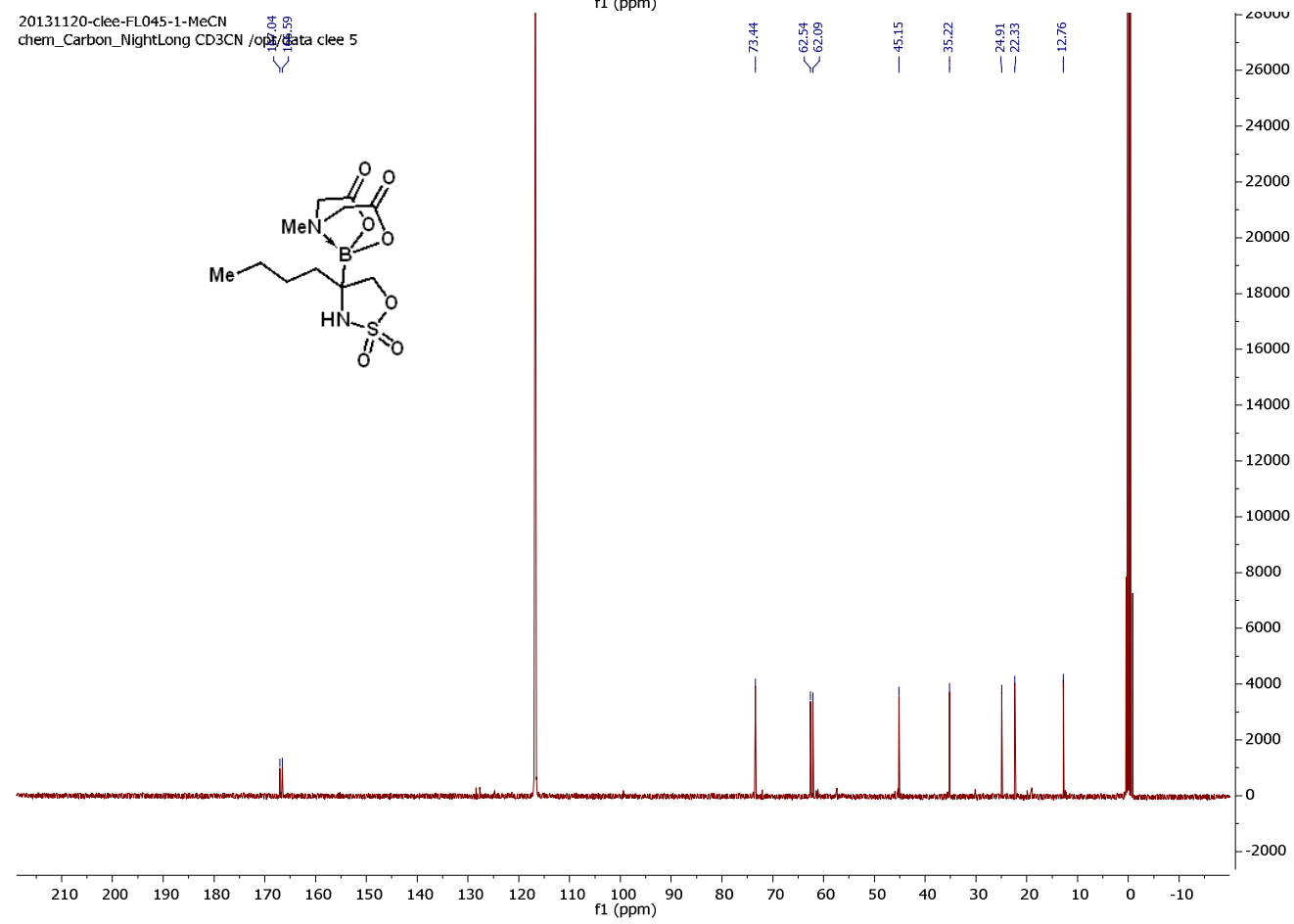


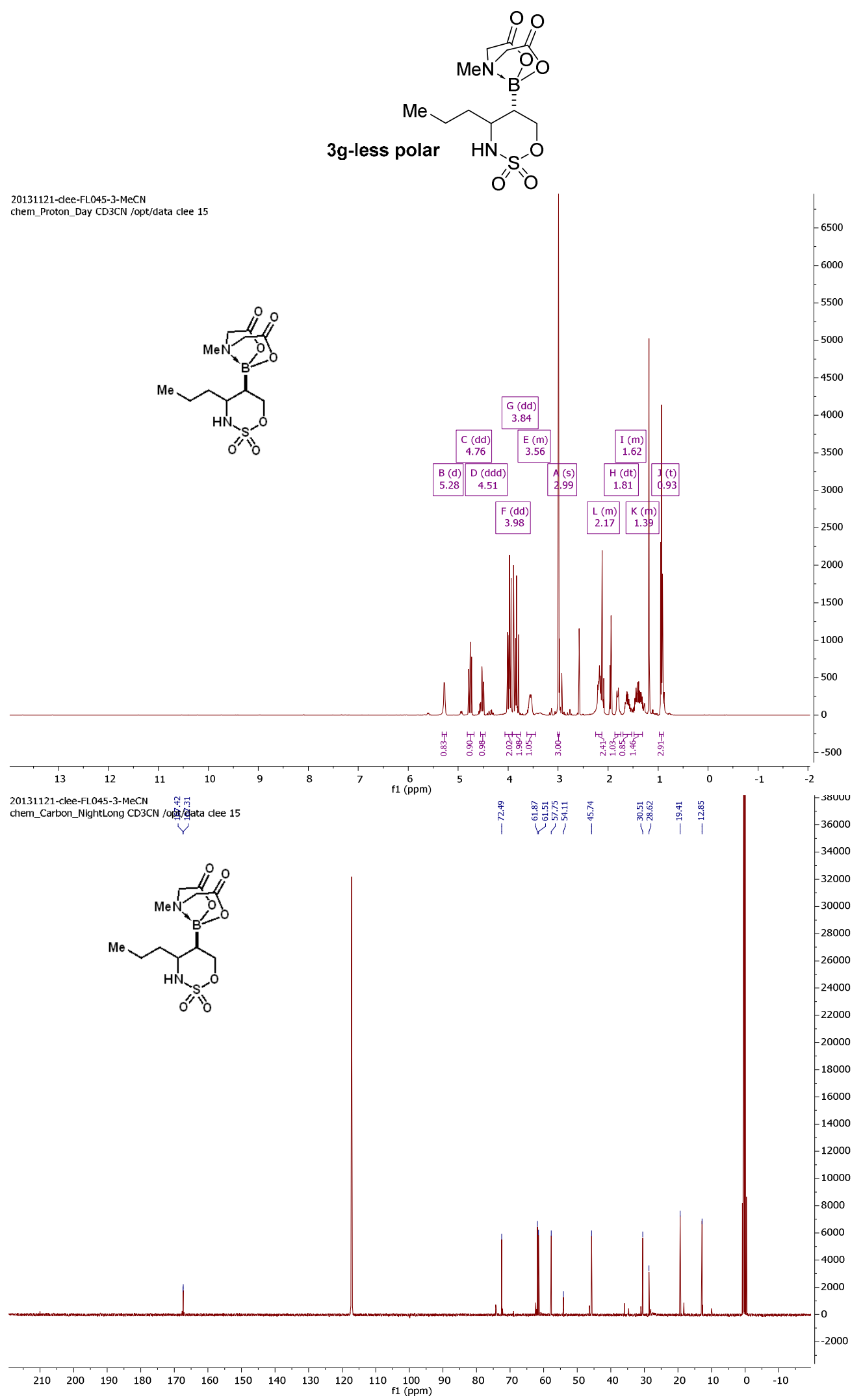




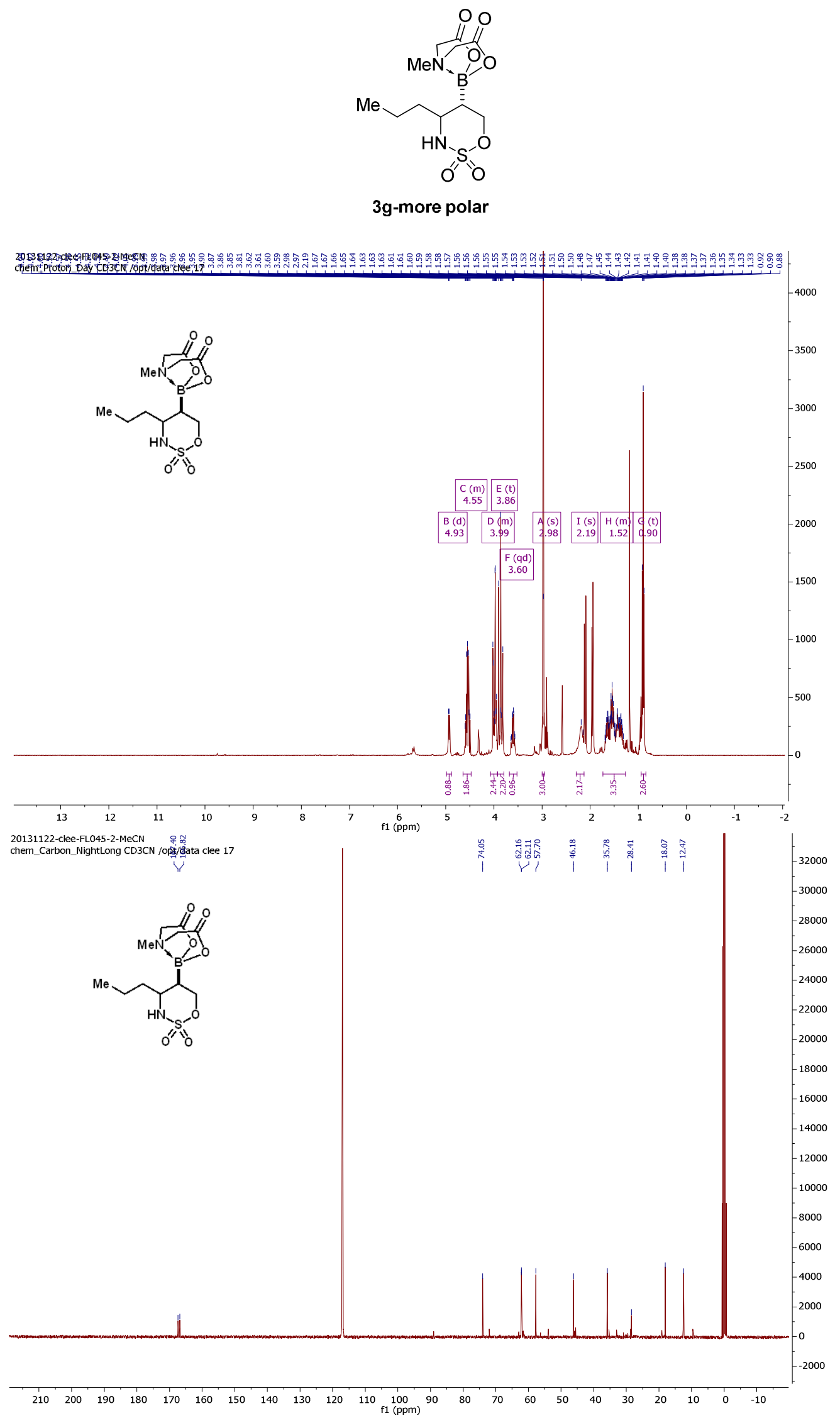



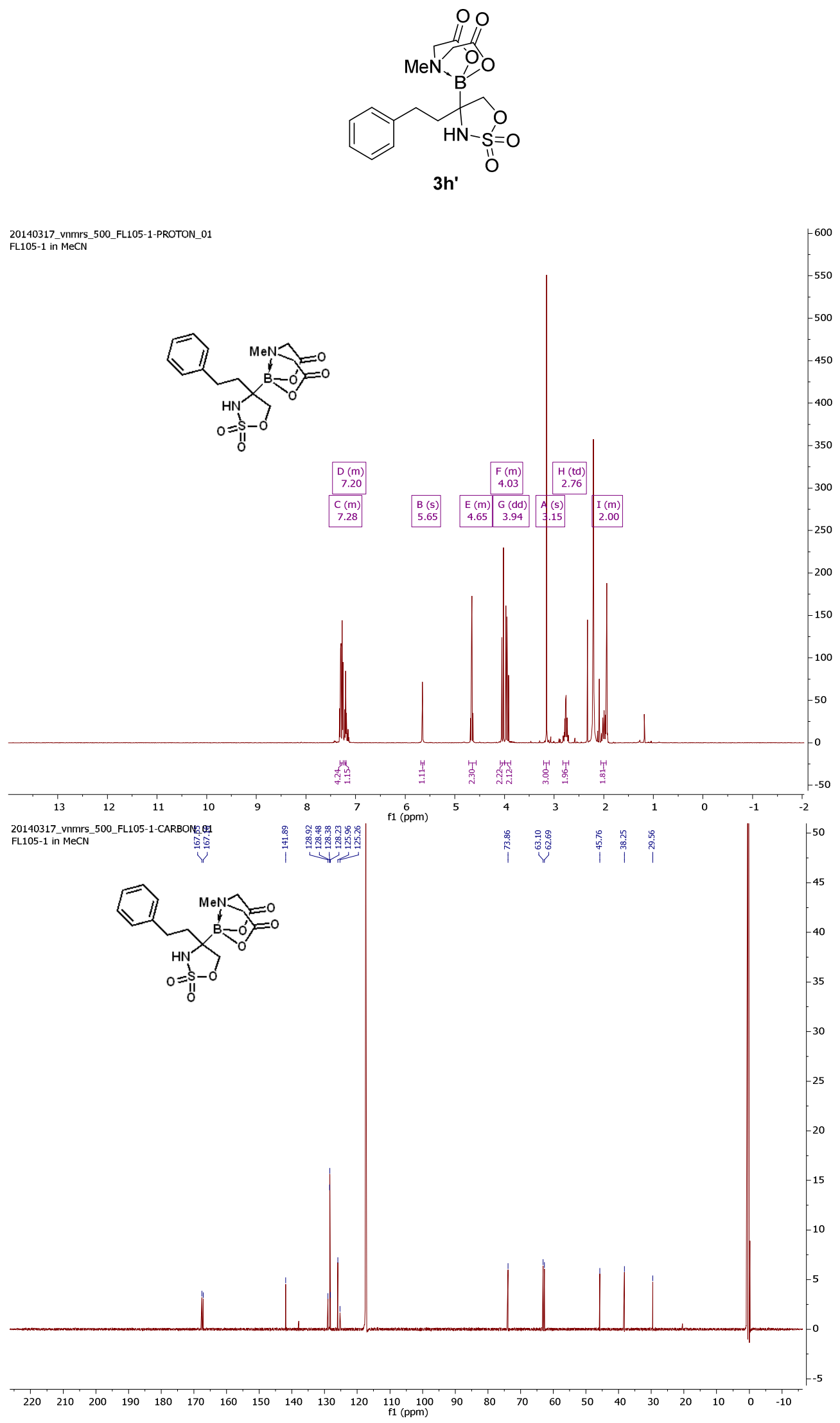


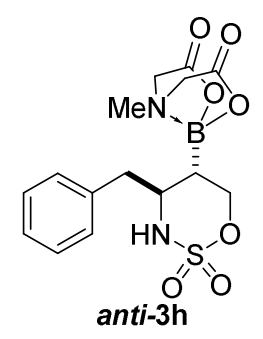

20140311_vnmrs_500_FL105-2-PROTON_01 FL105-2 in MeCN
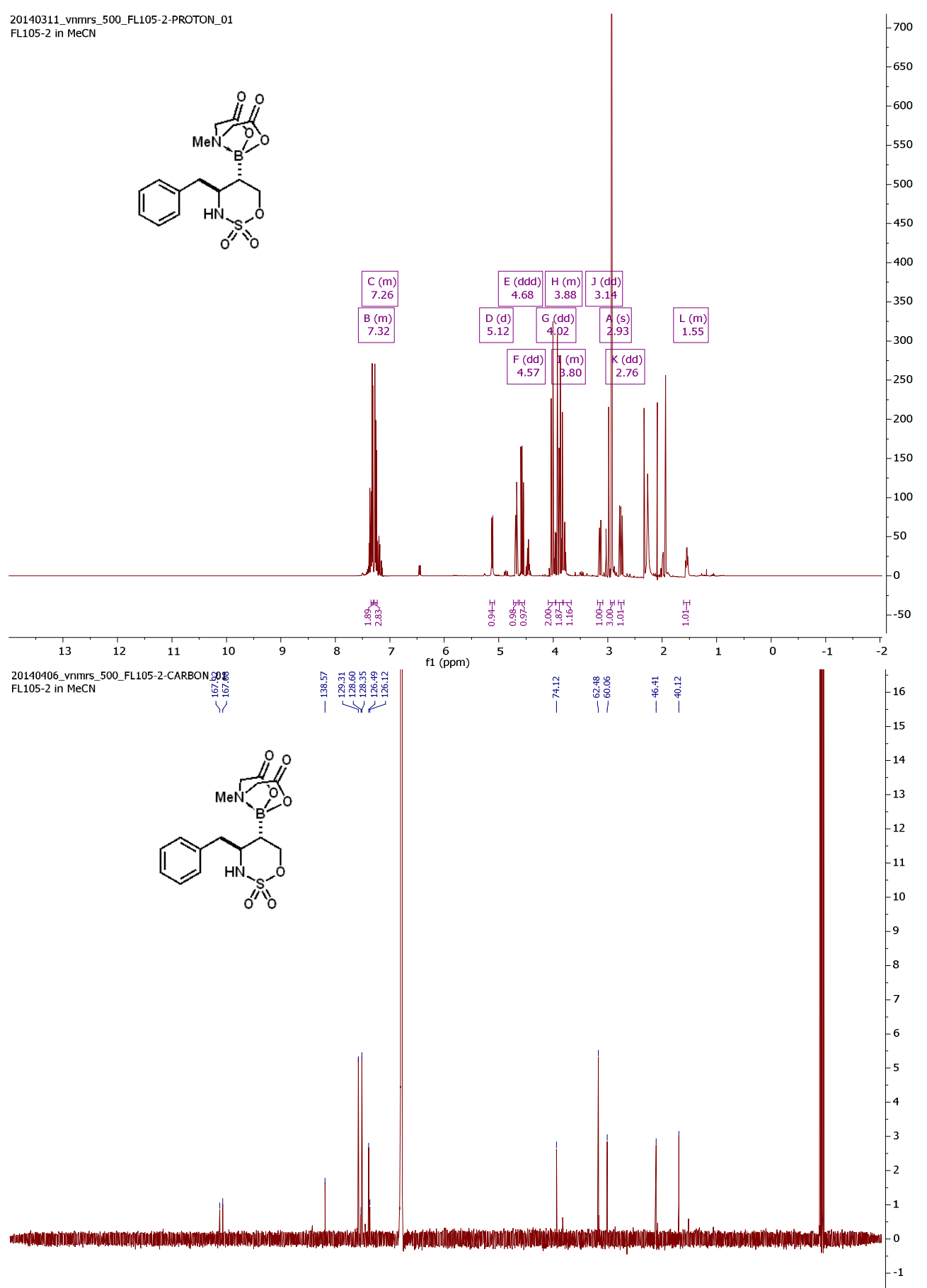

$\begin{array}{rlllllllllllllllllllllllll}1 & 220 & 210 & 200 & 190 & 180 & 170 & 160 & 150 & 140 & 130 & 120 & \underset{\mathrm{f} 1(\mathrm{ppm})}{110} & \mathbf{1 0 0} & 90 & 80 & 70 & 60 & 50 & 40 & 30 & 20 & 10 & 0 & -10\end{array}$ 


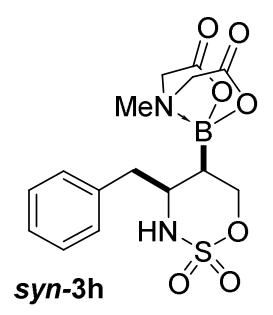

20140317_vnmrs_500_FL105-3-PROTON_01 FL105-3 in MeCN

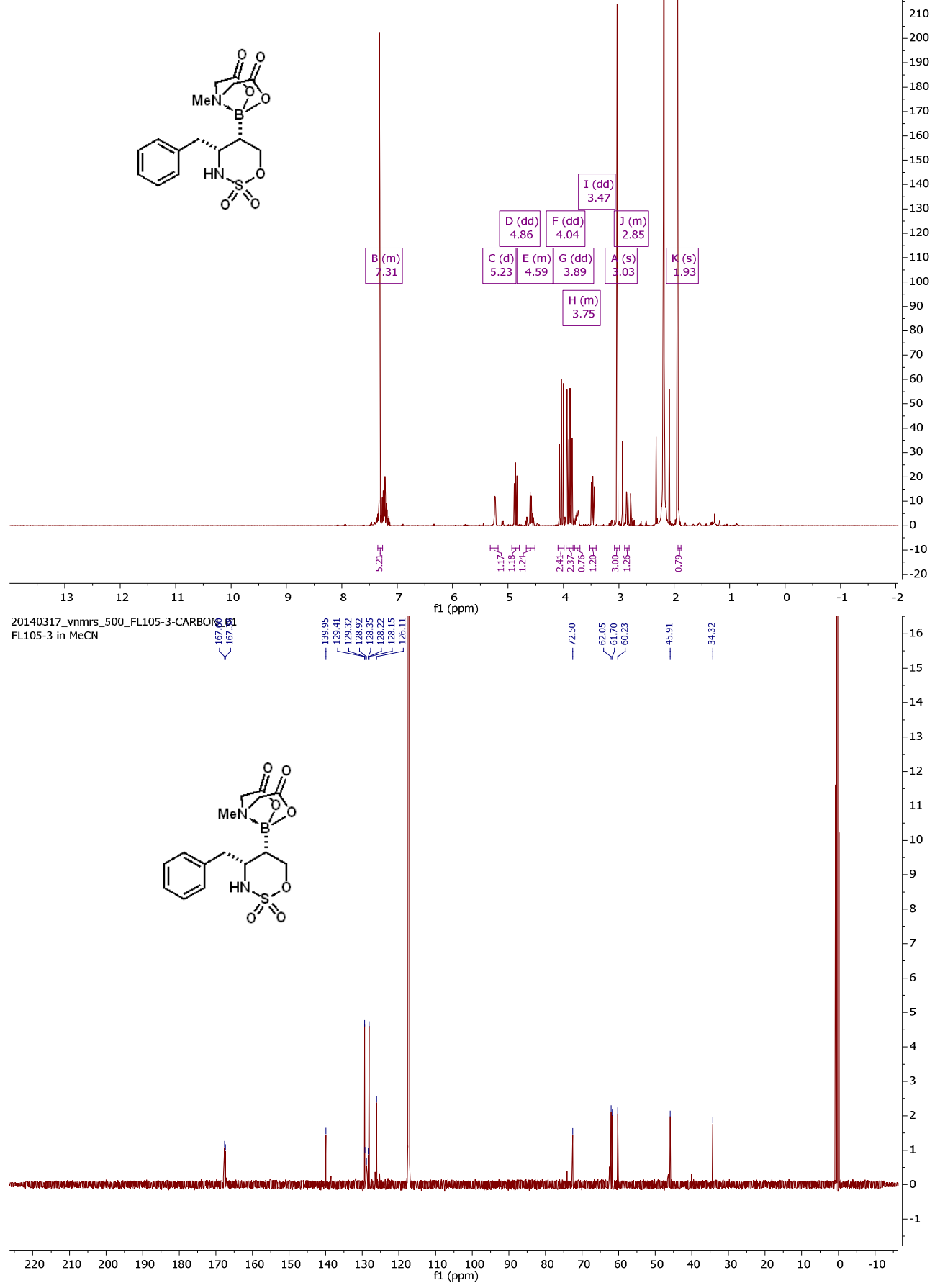




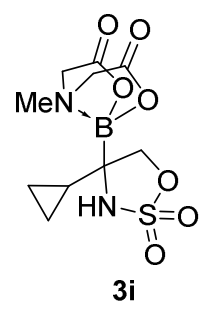

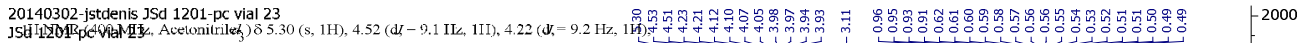

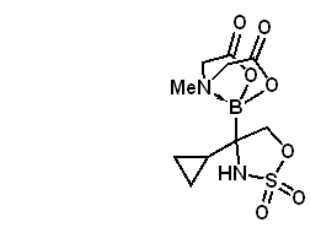

